Chapman University

Chapman University Digital Commons

Spring 5-2021

\title{
Bidimensional Assessment of Youth Mental Health: Evaluation of the California Healthy Kids Survey as a Measure of Youth Subjective Well-Being
}

Michael Doria

Chapman University, doria100@mail.chapman.edu

Follow this and additional works at: https://digitalcommons.chapman.edu/education_dissertations

Part of the Educational Psychology Commons

\section{Recommended Citation}

Doria, M. J. (2021). Bidimensional assessment of youth mental health: Evaluation of the California Healthy Kids Survey as a measure of youth subjective well-being [Doctoral dissertation, Chapman University]. Chapman University Digital Commons. https://doi.org/10.36837/chapman.000255

This Dissertation is brought to you for free and open access by the Dissertations and Theses at Chapman University Digital Commons. It has been accepted for inclusion in Education (PhD) Dissertations by an authorized administrator of Chapman University Digital Commons. For more information, please contact laughtin@chapman.edu. 


\title{
Bidimensional Assessment of Youth Mental Health:
}

\section{Evaluation of the California Healthy Kids Survey as a Measure of Youth Subjective Well-Being}

\author{
A Dissertation by \\ Michael J. Doria \\ Chapman University \\ Orange, $\mathrm{CA}$ \\ Attallah College of Educational Studies \\ Submitted in partial fulfillment of the requirements for the degree of \\ Doctor of Philosophy in Education
}

May 2021

Committee in charge:

Michael Hass, Ph.D., Chair

Kelly Kennedy, Ph.D.

John Brady, Ph.D. 
The dissertation of Michael J. Doria is approved.

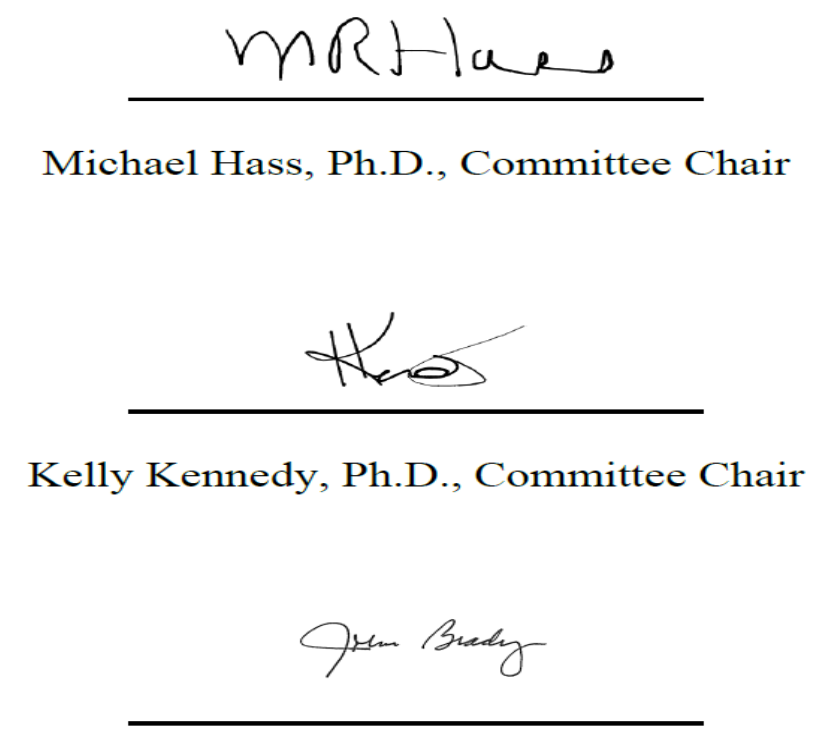

John Brady, Ph.D.

March 2021 


\section{Bidimensional Assessment of Youth Mental Health:}

Evaluation of the California Healthy Kids Survey as a Measure of Youth

Subjective Well-Being

Copyright (C) 2021

by Michael J. Doria 


\section{ACKNOWLEDGMENTS}

I have many people to thank who helped me along this journey. Finding an order to do so is not easy, given order is not a reflection of the level of my appreciation. That said, I will start by thanking my chair, Dr. Michael Hass, who has been a guiding support to me since I started at Chapman. As a professor, mentor, and friend, you have helped me to develop as a practitioner and researcher in this beautiful field of school psychology. I cannot thank you enough for your ongoing encouragement, countless hours spent discussing and editing my work, and the friendship along the way.

To Dr. Kelly Kennedy, thank you equally for your boundless support through this dissertation and my many years at Chapman. Your unparalleled expertise in methodology and statistics has been critically helpful. Thank you for the late evening hours running analyses with me and lending input for revisions.

I would like to also thank Dr. John Brady. From my early years at Chapman to now, you have been a valuable mentor in my development as school psychologist and researcher. I appreciate the support you have lent, from thoughtful edits to working out of the box to meet with me weekly at Starbucks to share your knowledge on systems evaluation.

To my parents, thank you for your unending love and support. You have both modeled for me the value of hard work, the need for compassion toward others, and the importance of pursuing your passions. With your influence, I find myself in a field I love, and that fills me with enthusiasm, which keeps me moving forward. I would not be here without the two of you. Thank you both for everything!

I would like to share my gratitude to my good friend Aaron Cullen who, along with Dr. Michael Hass, was instrumental in encouraging me to apply to the PhD program. Thank you, 
Aaron. Your encouragement, friendship, and time spent reading and sharing input on my work over the years has been guiding and simply enjoyed. I look forward to our continued exchanges on your research interests and hope, one day, I can talk you into making the same plunge into a doctoral program.

To my school psychology cohort, I consider myself uniquely privileged to have taken this journey alongside each of you. You individually have been a source of needed encouragement, humor, and meaningful collaboration. I sincerely hope we find time to continue writing and presenting together. I have endlessly benefitted from each of you. You are unquestionably among the most skilled clinicians, researchers, and visionaries in the field I have been privileged to know.

To Edward Reicks, $\mathrm{PhD}$, who fostered my love for psychology and encouraged me to pursue the field. Thank you for seeing in me what I did not see in myself. Your mentorship and friendship brought me to a career that has given me so much in my life. I am forever grateful for you.

Lastly, to my loving wife, Robin. I dedicate this dissertation to you and our daughter, Julianna. You have been a partner who has sustained equally, if not more, the sacrifice that came with pursing this degree. You are, and continue to be, my driving motivation for growth in all areas of life. Thank you for your love, patience, and encouragement through this part of our life journey together. I love you more than my words can convey! 


\begin{abstract}
Bidimensional Assessment of Youth Mental Health:

Evaluation of the California Healthy Kids Survey as a Measure of Youth

Subjective Well-Being

by Michael J. Doria
\end{abstract}

This study comprehensively analyzed extant data from the 2016-2017 California Healthy Kids Survey (CHKS) secondary core and Social Emotional Health Module (SEHM) to explore the ability of the CHKS to measure subjective well-being (SWB), a critical component in the assessment of youth mental health (MH). Research has validated the significance and utility of a dual continuum model of $\mathrm{MH}$, with SWB predicting emotional, behavioral, and cognitive engagement. Accordingly, when coupled with an assessment of psychopathology, positive evaluations of SWB can provide a more descriptive and comprehensive view of youth functioning than psychopathology alone. This more comprehensive view of students' functioning can inform asset-based interventions implemented in schools. Although there has been research validating the SEHM as a measure of SWB, no research to date has provided an evaluation of such for the CHKS Core. As the most widely used survey among the CHKS survey suite, this represented a critical gap. Results of Spearman correlations and point-biserial correlations between CHKS Core scales and the SEHM Covitality Index were analyzed for significance $(\alpha=$ .05). A significant positive relationship was established between each of CHKS Core subscales and SEHM Covitality Index. In addition, there were significant, inverse relationships between the CHKS Core subscales and psychopathology. In analysis of variance, the coefficient of 
determination indicated that limited variance between the analyzed scales correlated with covitality and psychopathology, could be accounted for. A categorical breakdown of frequencies and percentages for indicators of psychopathology and scales correlated with covitality, however, portrayed a meaningful relationship among variables. These findings suggested the CHKS Core has both concurrent validity in measurement of youth SWB and an inverse variablelevel relationship among SWB and psychopathology. As such, this study strengthens the utility of the CHKS Core as a measure of youth MH and holds the potential to enhance local education agencies' evaluation of student MH wellness to inform school MH programming. 


\section{TABLE OF CONTENTS}

$\underline{\text { Page }}$

ACKNOWLEDGMENTS ............................................................................................................ IV

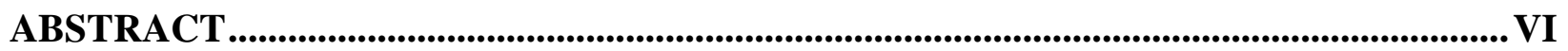

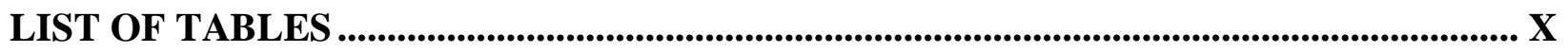

LIST OF FIGURES ...................................................................................................... XII

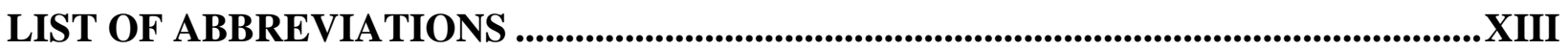

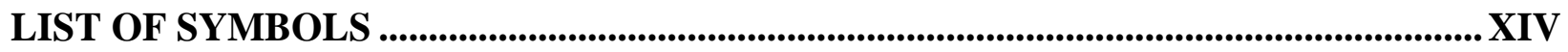

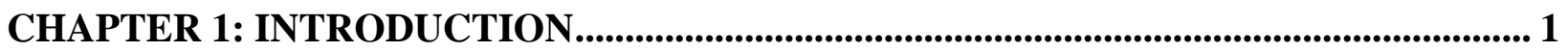

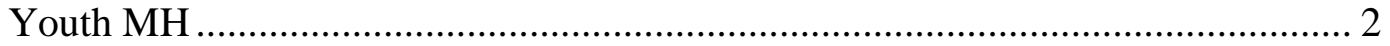

Asset-Based Model of MH ………………………......................................... 4

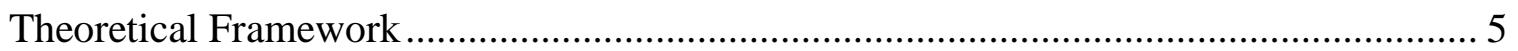

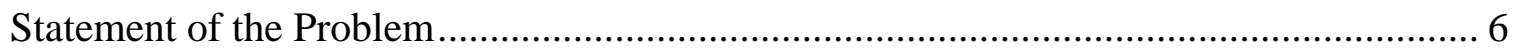

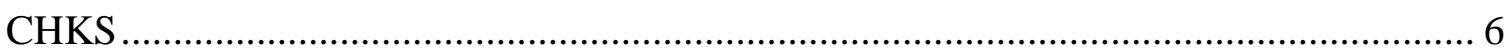

Purpose of the Present Study ................................................................................. 11

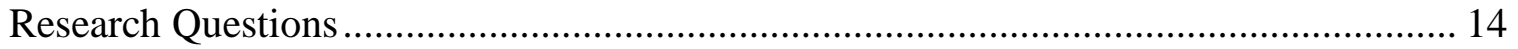

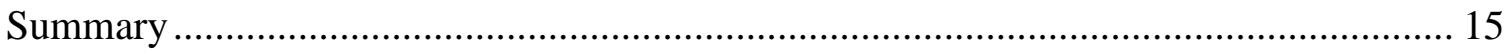

CHAPTER 2: LITERATURE REVIEW .................................................................................... 16

Subjective Well-Being ................................................................................ 16

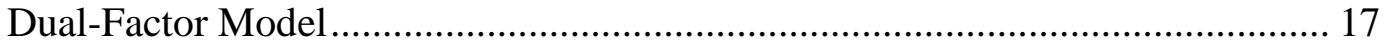

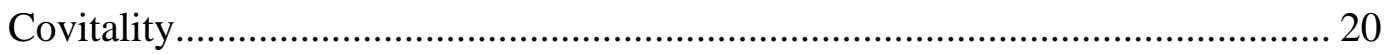

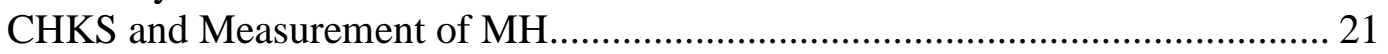

Risk Indicators of Psychopathology ……………………........................................... 23

Direct Indicators of Psychopathology and MH .............................................. 23

Indicators of SWB (External and Internal Assets) ……..................................... 25

CHKS Theoretical Alignment With SWB ………………....................................... 30

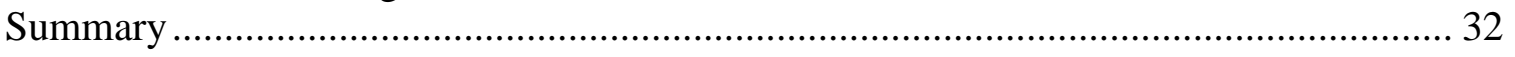

CHAPTER 3: RESEARCH METHODOLOGY _..................................................................... 33

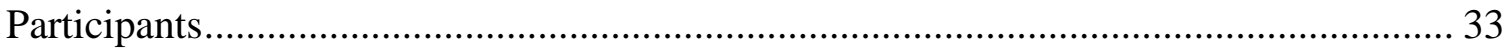

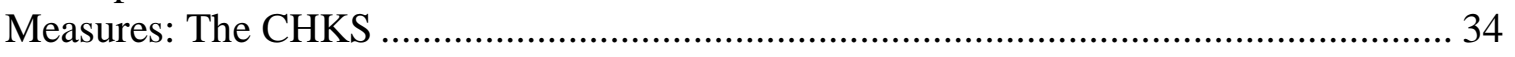

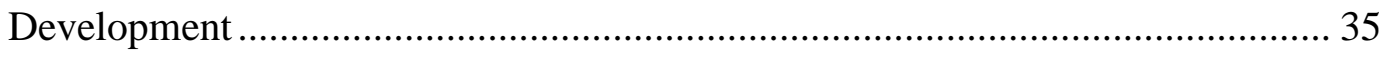

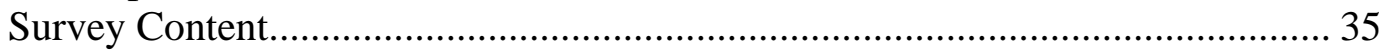

Reliability and Validity .............................................................................. 37

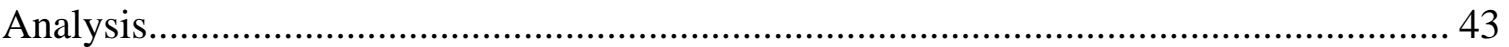

Research Question 1 .................................................................................... 43

Research Question 2 .................................................................................... 44 
Spearman Rank-Order Correlation............................................................................... 45

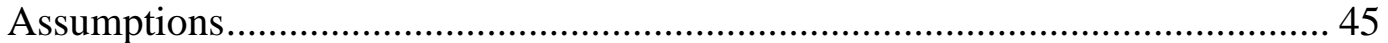

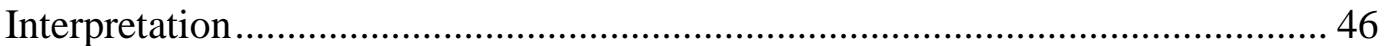

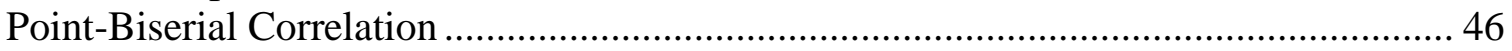

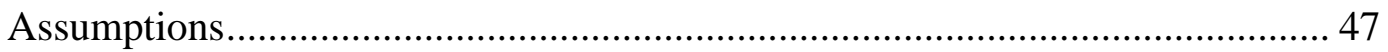

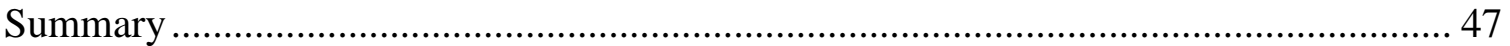

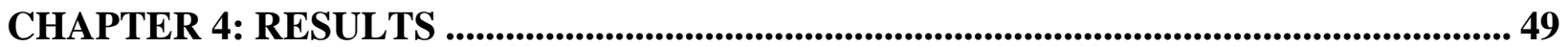

Frequencies and Percentages .................................................................................. 49

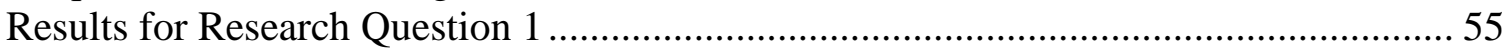

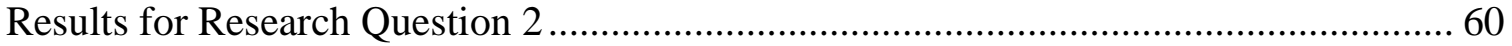

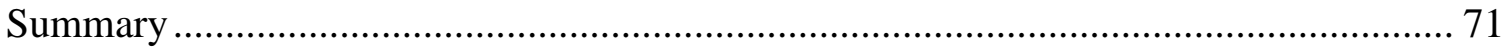

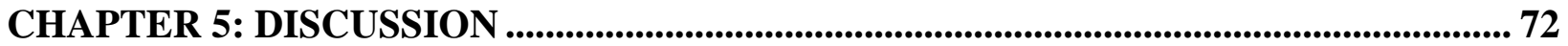

Discussion of Research Questions .............................................................................. 72

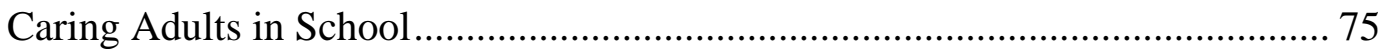

High Expectations-Adults ............................................................................. 78

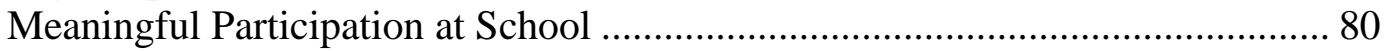

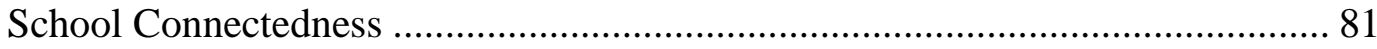

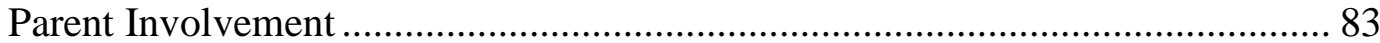

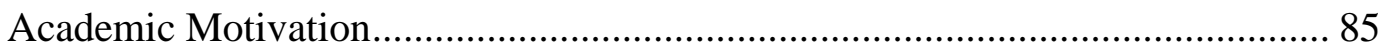

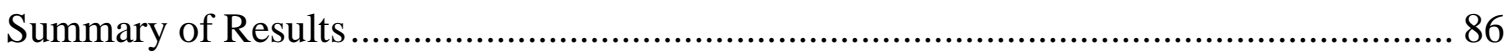

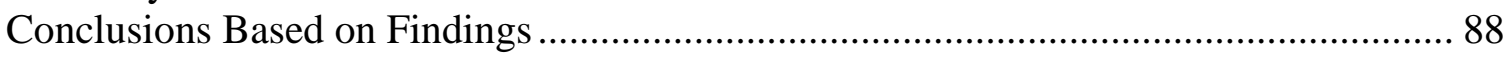

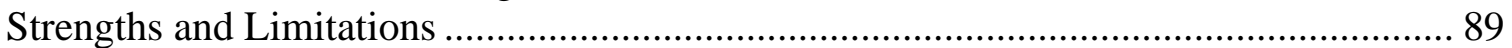

Missing Data, Mischievous Responders, and Self Report ................................... 89

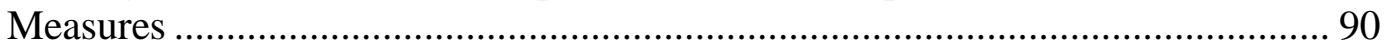

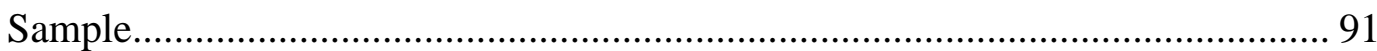

Methods of Analysis .................................................................................... 91

Recommendations for Future Research .................................................................... 92

Practical Implications....................................................................................... 94

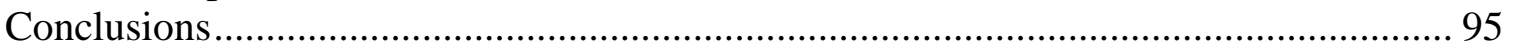

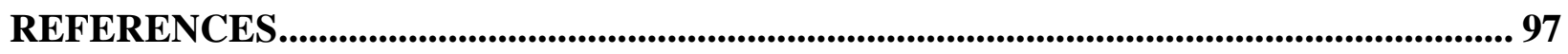

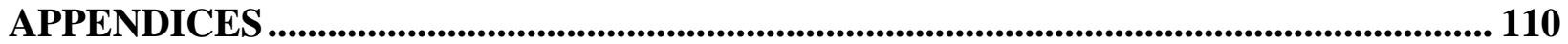




\section{LIST OF TABLES}

$\underline{\text { Page }}$

Table 1: CHKS and Mental Health Literature .................................................................... 8

Table 2: Dual Continua Model Class Membership................................................................ 19

Table 3: Frequencies and Percentages for Demographic Variables ....................................... 50

Table 4: Frequencies and Percentages for Sexual Orientation ............................................ 53

Table 5: Frequencies and Percentages for Reason for Missing School in Previous 30 days........ 54

Table 6: Descriptive Statistics for the Scales................................................................. 56

Table 7: Spearman Correlations Between the CHKS Core Subscales ..................................... 57

Table 8: Spearman Correlation Between Caring Adults in School and SEHM Covitality

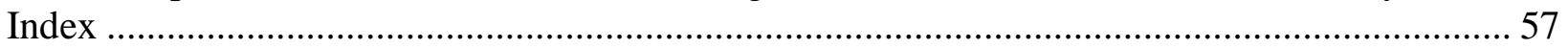

Table 9: Spearman Correlation Between High Expectations-Adults and SEHM Covitality

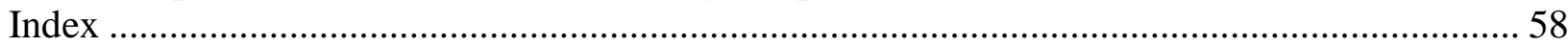

Table 10: Spearman Correlation Between Meaningful Participation at School and SEHM

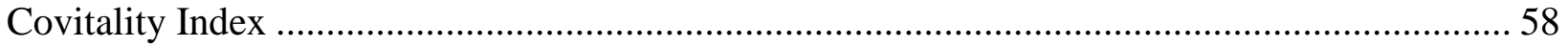

Table 11: Spearman Correlation Between School Connectedness and SEHM Covitality Index . 59

Table 12: Spearman Correlation Between Parental Involvement and SEHM Covitality Index ... 59

Table 13: Spearman Correlation Between Academic Motivation and SEHM Covitality Index .. 60

Table 14: Frequencies and Percentages for Indicators of Psychopathology ............................ 61

Table 15: Point-Biserial Correlation Between Very Sad, Hopeless, Anxious, Distressed, or Angry During Previous 12 Months and the CHKS Core Subscales

Table 16: Point-Biserial Correlation Between Feeling Sad or Hopeless Almost Every Day for 2 Weeks or More and the CHKS Core Subscales ................................................................... 63

Table 17: Point-Biserial Correlation Between Considering Attempting Suicide During Previous 12 Months and the CHKS Core Subscales the CHKS Core 64 
Table 18: Frequencies and Percentages for Indicators of Psychopathology by Caring Adults in School

Table 19: Frequencies and Percentages for Indicators of Psychopathology by High

Expectations-Adults

Table 20: Frequencies and Percentages for Indicators of Psychopathology by Meaningful Participation at School

Table 21: Frequencies and Percentages for Indicators of Psychopathology by School

Connectedness.

Table 22: Frequencies and Percentages for Indicators of Psychopathology by Parental Involvement

Table 23: Frequencies and Percentages for Indicators of Psychopathology by Academic Motivation 70 


\section{LIST OF FIGURES}

$\underline{\text { Page }}$

Figure 1: Positive Mental Health and Covitality Model Underlying the SEHM....................... 22

Figure 2: Role of Resilience and Psychological Well-Being in School Engagement ................. 31

Figure 3: Rejection Elements: Mischievous Responders................................................. 34

Figure 4: Theoretical and Measurement Model Underlying the SEHM................................ 40

Figure 5: Positive Mental Health and Covitality Model Underlying the SEHM....................... 42 


\section{LIST OF ABBREVIATIONS}

\begin{tabular}{|c|c|}
\hline Abbreviation & Meaning \\
\hline AOD & Alcohol and Other Drugs \\
\hline ANOVA & Analysis of Variance \\
\hline CSS & California Student Survey of Substance Use \\
\hline $\mathrm{CDE}$ & California Department of Education \\
\hline CFA & Confirmatory Factor Analysis \\
\hline CHKS & California Healthy Kids Survey \\
\hline DFM & Dual Factor Model \\
\hline IDEA & Individuals With Disabilities Education Act \\
\hline LEAs & Local Education Agencies \\
\hline MH & Mental Health \\
\hline MHSA & Mental Health Services Act \\
\hline PANAS-C & Positive and Negative Affectivity Scales for Children \\
\hline RYDM & Resilience Youth Development module \\
\hline SBMH & School-Based Mental Health \\
\hline SEHM & Social Emotional Health module \\
\hline SLSS & Student Life Satisfaction Scale \\
\hline SWB & Subjective Well-Being \\
\hline WHO & World Health Organization \\
\hline
\end{tabular}




\section{LIST OF SYMBOLS}

$\underline{\text { Symbol }} \underline{\text { Meaning }}$

$\alpha \quad$ Cronbach's alpha coefficient 


\section{CHAPTER 1: INTRODUCTION}

Although psychology provides varied models for diagnosis and treatment of mental health $(\mathrm{MH})$ disorders, there has been limited focus on psychological well-being as a distinct aspect of MH. Before the mid-20th century, MH models were firmly rooted in the medical model, which emphasized a biopsychological basis for MH disorders (Greenspoon \& Saklofske, 2001; Kinderman, 2005). Research in the field of positive psychology has highlighted positive aspects of observable MH. The construct of subjective well-being (SWB) evolved from this research. SWB has been defined as how individuals emotionally experience their lives (Diener, 1984).

More recently, the presence of SWB has been a critical factor in determining MH or wellness in youth (Antaramian et al., 2010; Diener, 2013; Greenspoon \& Saklofske, 2001; Kelly, 2012; Lyons et al., 2013; Renshaw \& Cohen, 2013). The evaluation of SWB holds value for educators and other stakeholders in school-based mental health (SBMH). Specifically, research has supported SWB as a predictor of all forms of student engagement (i.e., emotional, cognitive, and behavioral; Lyons et al., 2013). Moreover, when taken together with indicators of psychopathology, SWB provides a more comprehensive view of student MH functioning beyond psychopathology (Lyons, et al., 2013)). Research also has suggested a positive link between SWB and resilience, with resilience displaying predictive power for SWB (Rodriguez-Fernandez et al., 2018). The California Health Kids Survey (CHKS), a comprehensive health and resilience survey grounded in models of resilience by Benard (2004), shows promise in the evaluation of youth MH with consideration of their SWB. Lastly, other researchers have argued the CHKS shows promise in its ability to evaluate youth $\mathrm{MH}$ and assist with program evaluation and SBMH program planning (Austin \& O’Malley, 2012). Although promising in its alignment with a model 
of $\mathrm{MH}$ that includes SWB as one factor of wellness, an important question is if CHKS has validity as a measure of SWB.

A review of published research conducted with adolescents found only one study that specifically investigated the use of a dual continuum model of $\mathrm{MH}$, which emphasizes SWB, with CHKS's survey suite (Furlong et al., 2014). This represents a critical gap in the research literature and a limit on districts' ability to make informed decisions around student $\mathrm{MH}$ programming. Accordingly, this study focused on filling this gap by expanding research on CHKS's ability as a measure of youth MH by analyzing the CHKS high school core module as a measure of SWB.

\section{Youth MH}

The importance of this study is derived from the underserving of youth with $\mathrm{MH}$ needs. Youth $\mathrm{MH}$ is a growing concern highlighted by epidemiological data suggesting both high rates

of MH need and limited access to treatment (Burns et al., 1995; Merikangas et al., 2010; Weist et al., 2003). It has been estimated that 1 in every $4-5$ youths will have a diagnosable $\mathrm{MH}$ disorder marked by significant impairment or distress throughout their lifetime (Merikangas et al., 2010). Of this group, about 70\% have not accessed effective MH treatments (Adelman \& Taylor, 2012; Strein et al., 2003). Compared with other disabilities, MH disorders are identified late in life, often delaying access to support and potentially debilitating many children's ability to succeed in school (National Alliance on Mental Illness, n.d.).

Barriers to accessing $\mathrm{MH}$ treatment are significant (American Academy of Pediatrics [AAP], 2000; Reardon et al., 2017; Satcher, 2004). Barriers to MH servicers include inadequate insurance (AAP, 2000), insufficient financial ability to pay for services, challenges with transportation, an inadequate number of accessible child MH professionals, and concerns over 
stigmas related to MH disorders (Satcher, 2004). Other factors include perceived difficulty acquiring referrals, long wait times to access services, lack of confidence in care providers, perceived lack of interest, or blame coming from MH providers (Reardon, 2017). Finally, fragmentation of services resulting in narrowly focused interventions operating in isolation from one another presents a significant barrier in both schools and community MH organizations (Adelman \& Taylor, 2012).

A broad national consensus supports the importance of schools in meeting MH needs of youth. For example, the Individuals With Disabilities Education Act (2004) stipulated children with emotional and behavioral disorders must receive an education that prepares them for optimal intellectual, occupational, and social functioning as adults. The President's Freedom Commission on Mental Health (2003) also recommended

quality screening and early intervention will occur in both readily accessible, low-stigma settings, such as primary health care facilities and schools, and in settings in which a high level of risk exists for MH problems, such as criminal justice, juvenile justice, and child welfare systems. ( $\sec 3.2)$

Additionally, the No Child Left Behind Act of 2001, later reauthorized as the Every Student Succeeds Act (2015), stated schools must provide "student access to quality mental health care by developing innovative programs to link the local school system with the mental health system."

In California, the Mental Health Services Act (MHSA) of 2004 called for implementing comprehensive community-based MH services and supports. With this, the MHSA called for implementing comprehensive MH services starting from prekindergarten and continuing through 12th grade. This legislation aims to decrease the overall long-term adverse effects on both 
individuals and families who confront problems with $\mathrm{MH}$. By expanding innovative services that demonstrate efficacy through the support of state and local funds, the MHSA supports prevention and intervention earlier in life than was traditionally observed.

Schools have become de facto MH systems for the limited number of youth who receive MH services (Burns et al., 1995). Data from several studies suggested $70 \%-80 \%$ of those who receive MH services access them in school (Adelman \& Taylor, 2012; Burns et al., 1995; Wiley \& Corey, 2013). Kaplan et al. (1998) found adolescents were 10 times more likely to access MH services if they had access to school-based MH services. Given this, schools have a critical role in addressing student MH needs. Consequently, expectations for schools to provide access to MH services have increased in kind (Individuals With Disabilities Education Act [IDEA], 2004; President's New Freedom Commission on Mental Health, 2003). Along with expectations that schools provide MH services for students, there now is a concurrent expectation that school MH programs' efficacy will be critically evaluated.

\section{Asset-Based Model of MH}

In this evaluation of school MH programs, a strength-based approach to bolstering positive student outcomes is crucial. It is well established that the promotion of positive MH is a fundamental aspect to bolstering general student well-being (Institute of Medicine, 2009).

Further, ongoing research has established that the sheer absence of psychopathology is not a firm guarantor for positive well-being (Antaramian et al., 2010; Furlong et al., 2014; Greenspoon \& Saklofske, 2001; Jahoda, 1958; Jones et al., 2013; Kelly et al., 2012; Kinderman, 2005; Lyons et al., 2013; Ryff \& Singer, 1998; Suldo \& Schaffer, 2008). Instead, SWB, how an individual emotionally experiences their life (Diener, 1984), has become the final critical determinant in 
characterizing wellness (Antaramian et al., 2010; Diener, 2013; Greenspoon \& Saklofske, 2001; Kelly, 2012; Renshaw \& Cohen, 2013; Suldo \& Shaffer, 2008; Suldo et al., 2016).

In assessing youth $\mathrm{MH}$, two separate models have been responsible for expanding our conceptualization of MH as inclusive of the presence of SWB. The dual-factor model (DFM) and covitality models of MH (Furlong et al., 2014; Suldo \& Shaffer, 2008) together comprise two different expressions of the modern bidimensional conceptualization of wellness. These two asset-based models have significant utility for youth MH beyond the simple evaluation of student functioning. For school MH programming, identifying internal and external assets associated with well-being is vital to supporting the development and evaluation of district-wide $\mathrm{MH}$ programs.

\section{Theoretical Framework}

To assess these factors, one must understand the theoretical framework of a bidimensional model of $\mathrm{MH}$. Through advances in research in the field of positive psychology, researchers have accepted widely that the absence of psychopathology, otherwise referred to as mental illness, is not sufficient for determining MH (Jahoda, 1958). Instead, MH is best measured by assessing the presence or absence of psychopathology combined with positive indicators such as self-acceptance, social contribution, and life satisfaction (Ryff \& Singer, 1998). A bidimensional model conceptualizes MH as the result of the interaction of two separate factors: psychopathology and SWB (Greenspoon \& Salklofske, 2001).

SWB, the critical addition to this model of MH, refers to "how people experience and evaluate their lives" (National Research Council, 2013, p. 1). The evaluation of the construct of SWB includes both cognitive and affective evaluations of one's life. These include three components: (a) positive affect, (b) negative affect, and (c) life satisfaction (Antaramian et al., 
2010). In the assessment of youth MH, DFM and covitality models generate an evaluation of SWB and psychopathology. For this study, the covitality model established by Furlong et al. $(2013,2014)$ and validated in the SEHM within the CHKS survey suite was used as a measure of SWB consistent with the theoretical framework of a bidimensional model of $\mathrm{MH}$.

\section{Statement of the Problem}

Legislative mandates have called for school involvement in student MH by requiring school districts to take an active approach in student MH support (Every Student Succeeds Act, 2015; IDEA, 2004; President's Freedom Commission on Mental Health, 2003). This strategy agrees with school MH literature, which explicitly advocates for school involvement at the local level (Adleman \& Taylor, 2012). However, this approach requires ongoing and regular assessment of student MH needs, repetitive evaluation of school and community resources, and functional evaluation of efficacy in SBMH supports.

\section{CHKS}

One method of assessment that has the potential to provide critical information to LEAs is the CHKS, a "comprehensive youth health and resilience survey" (WestEd, n.d.-a, para. 1) employed statewide by the California Department of Education (CDE) through a nonprofit educational group, WestEd. This survey is required to be administered by LEAs biennially to receive Title IV funds (WestEd, n.d.-a). The purpose of the CHKS is to "provide data to guide efforts to reduce student health risk behaviors and build schools that are a drug-free, safe and supportive haven for healthy positive youth development and achievement" (Austin \& Duerr, 2004, p. 1). A self-report survey of youth, the CHKS, is focused on evaluating significant characteristics of health-related risk, behavior, and resilience (WestEd, n.d.-a). Both the elementary and secondary survey suites each consist of a core module comprised of a set of 
supplemental modules that allow LEAs to customize their data collection based on areas of need for their unique population. For this study, data from the 2016-2017 secondary CHKS Core and SEHM were analyzed (see Appendix A).

Austin and O'Malley (2012) have argued the CHKS is a helpful guide for school districts and community stakeholders to plan prevention and intervention services for youth and, thus, holds promise for its application to youth MH (Austin \& O’Malley, 2012; WestEd, n.d.-a). The CHKS has shown the potential to function as an MH program planning tool. The CHKS Core and supplemental survey models illustrate this by assessing aspects of youth MH wellness that show an established connection to school-related outcomes (Austin \& O’Malley, 2012).

These outcomes include grades, attendance, and graduation. Factors such as school climate, feelings of physical and social-emotional safety, connectedness to peers and staff, the existence of supportive relationships, chances for meaningful participation and contribution, high expectations from others, and substance use are among some of the additional critical variables related to student MH wellness assessed by the CHKS. Other items valued are indicators of psychopathology, such as depression, stress, and anxiety. These outcomes areas related to student MH give fair data for identifying psychopathology and some general assessment of wellbeing but would require more validated items for assessing student SWB to align appropriately with the bidimensional model of MH.

Given the literature on CHKS's alignment with a bidimensional model of MH wellness, Furlong et al. (2014) validated the SEHM, a supplemental module LEAs can select to implement in the application of a bidimensional model of MH. In their research, Furlong et al. established a theoretical model of 12 first-order constructs that collectively load onto a metaconstruct labeled 
covitality. Importantly, through path modeling, Furlong et al. found covitality was a strong predictor of SWB.

Although there is literature on the validation and application of the covitality model and its ability to assess student SWB, no research to date has investigated the CHKS Core survey's utility to measure student MH consistent with the bidimensional framework by assessing SWB (see Table 1). In addition to Furlong et al. (2014), only one other study using the CHKS has been conducted to evaluate a comprehensive model (Castro-Olivo et al., 2013). The study, however, focused on using CHKS to validate model fit for theories of intervention to explore two resiliency building and violence prevention models for their effectiveness (Castro-Olivo et al., 2003). The study did not evaluate CHKS for any properties related to the evaluation of SWB.

\section{Table 1}

\section{CHKS and Mental Health Literature}

\begin{tabular}{|c|c|c|}
\hline Citation & Topic & Analysis \\
\hline $\begin{array}{l}\text { Baams et al., } \\
2017\end{array}$ & $\begin{array}{l}\text { Link between bias-based bullying, absenteeism due to } \\
\text { feelings of safety at school, and reduced school funding } \\
\text { in California. }\end{array}$ & $\begin{array}{l}\text { Pearson's correlation; } \\
\text { logistics regressions }\end{array}$ \\
\hline Banato, 2011 & $\begin{array}{l}\text { The relationship of external school protective factors and } \\
\text { internal student assets with student academic } \\
\text { achievement. }\end{array}$ & $\begin{array}{l}\text { Correlation; hierarchical } \\
\text { multiple regression }\end{array}$ \\
\hline $\begin{array}{l}\text { Benbenishty et } \\
\text { al., } 2016\end{array}$ & $\begin{array}{l}\text { The direct relationship among school climate, violence, } \\
\text { and academic performance over time. }\end{array}$ & $\begin{array}{l}\text { Structural equation } \\
\text { modeling }\end{array}$ \\
\hline $\begin{array}{l}\text { Benbenishty et } \\
\text { al., } 2017\end{array}$ & $\begin{array}{l}\text { School differences for suicide ideation and the influence } \\
\text { role of student characteristics, school- attributes. }\end{array}$ & Multilevel analysis \\
\hline $\begin{array}{l}\text { Bersamin et al., } \\
2017\end{array}$ & $\begin{array}{l}\text { Variance in the relationship between access to school- } \\
\text { based health centers and adolescent substance abuse by } \\
\text { race/ethnicity, sex, and socioeconomic status. }\end{array}$ & $\begin{array}{l}\text { Descriptive statistics; } \\
\text { multilevel logistic regression }\end{array}$ \\
\hline $\begin{array}{l}\text { Boston \& } \\
\text { Warren, } 2017\end{array}$ & $\begin{array}{l}\text { Effects of belonging and racial identity on urban African } \\
\text { American high school students' achievement. }\end{array}$ & $\begin{array}{l}\text { Correlation; multiple } \\
\text { regression analysis }\end{array}$ \\
\hline
\end{tabular}




\begin{tabular}{|c|c|c|}
\hline Citation & Topic & Analysis \\
\hline $\begin{array}{l}\text { Capp et al., } \\
2016\end{array}$ & $\begin{array}{l}\text { The influence of relationships from multiple contexts for } \\
\text { adolescents and their MH. }\end{array}$ & $\begin{array}{l}\text { Ordinary least squares } \\
\text { regression }\end{array}$ \\
\hline $\begin{array}{l}\text { Castro-Olivo et } \\
\text { al., } 2013\end{array}$ & $\begin{array}{l}\text { Evaluation of resilience building/violence prevention } \\
\text { models. }\end{array}$ & $\begin{array}{l}\text { Structural equation } \\
\text { modeling }\end{array}$ \\
\hline $\begin{array}{l}\text { De Pedro et al., } \\
2017\end{array}$ & $\begin{array}{l}\text { Occurrence of substance use in transgender youth } \\
\text { compared to non-transgender youth. }\end{array}$ & $\begin{array}{l}\text { Bivariate analysis; } \\
\text { multivariate analysis; chi- } \\
\text { square; multivariate } \\
\text { regression; logistics } \\
\text { regression }\end{array}$ \\
\hline $\begin{array}{l}\text { De Pedro et al., } \\
2016\end{array}$ & $\begin{array}{l}\text { Latent class analysis of school climate among middle and } \\
\text { high school students in California public schools. }\end{array}$ & $\begin{array}{l}\text { Latent class analysis; } \\
\text { multinomial logistic } \\
\text { regression' }\end{array}$ \\
\hline $\begin{array}{l}\text { Davidson-Arad } \\
\text { \& Navaro- } \\
\text { Bitton, } 2015\end{array}$ & $\begin{array}{l}\text { Levels and predictors of resilience of maltreated } \\
\text { adolescents in foster care with those of maltreated } \\
\text { adolescents in residential and community care. }\end{array}$ & $\begin{array}{l}\text { One-way analysis of variance } \\
\text { (ANOVA); analysis of } \\
\text { standard deviation and F } \\
\text { value; post hoc Scheffe test; } \\
\text { Pearson correlation; two-way } \\
\text { ANOVA; hierarchical } \\
\text { regression }\end{array}$ \\
\hline $\begin{array}{l}\text { Davis et al., } \\
2014\end{array}$ & $\begin{array}{l}\text { Association among victimization from bullying, suicide, } \\
\text { hopelessness, and the existence of a Gay-Straight } \\
\text { Alliance on a school campus. }\end{array}$ & Hierarchical modeling \\
\hline Day et al., 2018 & $\begin{array}{l}\text { Analysis of gender identity-related disparities in school } \\
\text { experiences (i.e., absenteeism, victimization and } \\
\text { harassment, and academic success) and perceptions of } \\
\text { school climate, reasons youth are truant from school, and } \\
\text { gender identification and school connectedness. }\end{array}$ & $\begin{array}{l}\text { Logistics regression; } \\
\text { multilevel regression }\end{array}$ \\
\hline Day et al., 2016 & $\begin{array}{l}\text { Effects of support, nonpunitive, practices on homophobic } \\
\text { bullying and school connectedness. }\end{array}$ & Multilevel modeling \\
\hline $\begin{array}{l}\text { Estrada et al., } \\
2017\end{array}$ & $\begin{array}{l}\text { Relationship among school violence, military connection, } \\
\text { and gang membership. }\end{array}$ & $\begin{array}{l}\text { Frequency distribution; } \\
\text { logistics regression }\end{array}$ \\
\hline $\begin{array}{l}\text { Ferguson \& } \\
\text { Xie, } 2012\end{array}$ & $\begin{array}{l}\text { Adult support and substance use among homeless youth } \\
\text { attending high school. }\end{array}$ & $\begin{array}{l}\text { Global empirical analysis; } \\
\text { structural equation modeling; } \\
\text { confirmatory factor analysis } \\
\text { (CFA) }\end{array}$ \\
\hline $\begin{array}{l}\text { Furlong et al., } \\
2009\end{array}$ & $\begin{array}{l}\text { Evaluate and enhance the relevance of the Resilience } \\
\text { Youth Development module (RYDM) of CHKS for } \\
\text { practicing school psychologists. }\end{array}$ & $\begin{array}{l}\text { Monovalent; One-way } \\
\text { ANOVA; correlation }\end{array}$ \\
\hline $\begin{array}{l}\text { Furlong et al., } \\
2014\end{array}$ & $\begin{array}{l}\text { Reports on preliminary development and validation of the } \\
\text { SEHM. }\end{array}$ & $\begin{array}{l}\text { CFA; invariance analysis; } \\
\text { latent means testing }\end{array}$ \\
\hline
\end{tabular}




\begin{tabular}{|c|c|c|}
\hline Citation & Topic & Analysis \\
\hline $\begin{array}{l}\text { Gilreath et al., } \\
2014\end{array}$ & Substance use among California adolescents. & Latent class analysis \\
\hline $\begin{array}{l}\text { Larson et al., } \\
2019\end{array}$ & $\begin{array}{l}\text { School victimization in secondary students and related } \\
\text { health outcomes that may adversely impact academic } \\
\text { outcomes. }\end{array}$ & Descriptive statistics \\
\hline $\begin{array}{l}\text { Lenzi et al., } \\
2017\end{array}$ & $\begin{array}{l}\text { Relationship among sense of community and teacher } \\
\text { support; two components of school climate. }\end{array}$ & Multilevel regression \\
\hline $\begin{array}{l}\text { Lenzi et al., } \\
2015\end{array}$ & $\begin{array}{l}\text { The relationship among the amount and variety of } \\
\text { developmental assets and school victimization in youth. }\end{array}$ & $\begin{array}{l}\text { Chi-square; mixed effects } \\
\text { modeling; omnibus test }\end{array}$ \\
\hline $\begin{array}{l}\text { Loeb et al., } \\
2014\end{array}$ & $\begin{array}{l}\text { The association of high expectations communicated in the } \\
\text { community, home, and school, victimization and dating } \\
\text { violence victimization, and peer norms. }\end{array}$ & $\begin{array}{l}\text { Univariate analysis; bivariate } \\
\text { analysis; logistics regression }\end{array}$ \\
\hline $\begin{array}{l}\text { Midford et al., } \\
2017\end{array}$ & $\begin{array}{l}\text { Evaluation of an evidence based social-emotional } \\
\text { learning program. }\end{array}$ & Paired $t$ test \\
\hline $\begin{array}{l}\text { Perez-Brummer } \\
\text { et al., } 2017\end{array}$ & $\begin{array}{l}\text { Examination of gender identity-related disparities in } \\
\text { prevalence of suicidal ideation. }\end{array}$ & $\begin{array}{l}\text { Multivariate logistics } \\
\text { regression }\end{array}$ \\
\hline $\begin{array}{l}\text { Shim-Pelayo \& } \\
\text { De Pedro, } 2018\end{array}$ & $\begin{array}{l}\text { California foster care youth and the association of school } \\
\text { climate in the occurrence of depression and suicidal } \\
\text { ideation. }\end{array}$ & $\begin{array}{l}\text { Bivariate analysis (chi- } \\
\text { square); multivariate logistics } \\
\text { regression }\end{array}$ \\
\hline $\begin{array}{l}\text { Stone et al., } \\
2013\end{array}$ & $\begin{array}{l}\text { Association among school-based health centers and } \\
\text { student-reported school assets. }\end{array}$ & Propensity scoring \\
\hline $\begin{array}{l}\text { Sullivan et al., } \\
2018\end{array}$ & $\begin{array}{l}\text { Association of parent identification indicators with } \\
\text { substance use patterns among military connected } \\
\text { adolescents. }\end{array}$ & Latent class analysis \\
\hline $\begin{array}{l}\text { Voight et al., } \\
2013\end{array}$ & $\begin{array}{l}\text { Evaluation of differences in school climate by students in } \\
\text { successful versus unsuccessful schools. }\end{array}$ & Descriptive statistics \\
\hline $\begin{array}{l}\text { Voight \& } \\
\text { Hanson, } 2017\end{array}$ & $\begin{array}{l}\text { How are middle school climate and academic } \\
\text { performance related across schools and over time. }\end{array}$ & Multilevel regression \\
\hline $\begin{array}{l}\text { Waters \& } \\
\text { Cross, } 2010\end{array}$ & $\begin{array}{l}\text { Evaluative characteristics of three composite scales of } \\
\text { adolescent connectedness, adapted from Add Health } \\
\text { study and CHKS. }\end{array}$ & $\begin{array}{l}\text { Exploratory factor analysis; } \\
\text { Cronbach alpha; congeneric } \\
\text { modeling }\end{array}$ \\
\hline
\end{tabular}

Consequently, although there has been research validating the covitality model as a measure of SWB, no study to date has explored a similar utility in the CHKS Core. Specifically, although there is research and theory asserting the CHKS Core module assesses aspects of 
student psychopathology (Austin \& O’Malley, 2012), a critical factor in the dual continuum model of $\mathrm{MH}$, there are no studies that explore the CHKS Core as a measure of student SWB. Even though CHKS SEHM potentiality fills this need, data from West Ed (n.d.-a) illustrate the CHKS Core is the most widely used module among school districts. As the most commonly used CHKS module, lack of research on the core module represented a critical gap in the literature. Research on the core module can assist stakeholders in planning and evaluating school $\mathrm{MH}$ programs.

\section{Purpose of the Present Study}

Accordingly, to fill this gap, this study focused on comprehensively analyzing the CHKS secondary core survey module to explore its ability to measure SWB, a critical component to assessing youth $\mathrm{MH}$ consistent with the dual continuum theory. The research supporting the utility and relevance of a dual continuum model of $\mathrm{MH}$, with SWB predicting all forms of student engagement (i.e., emotional, behavioral, and cognitive; Lyons et al., 2013), has clarified the utility of this model for school MH stakeholders. Moreover, when coupled with an assessment of psychopathology, evaluations of SWB can provide a more comprehensive view of youth functioning than psychopathology alone. This more comprehensive view of students' functioning can inform asset-based interventions such as social-emotional learning programs implemented in schools. This study expanded on prior research by assessing student outcomes, psychopathology, and SWB using the CHKS Core module. The CHKS Core demonstrated an ability to identify factors related to student SWB, so this study contributed to the literature by identifying correlations between SWB and student outcomes.

Validity is an important factor in establishing a measure's ability to appropriately assess a construct. Consequently, in evaluating the CHKS Core's ability to measure SWB, it was 
established that the CHKS Core is a valid tool for assessing student SWB. As a basic concept, validity refers to the "extent to which a test measures what it claims to measure" (Maul, 2018, p. 2). Although the CHKS does not currently assert to measure SWB, it performs as a youth resilience measure, evaluating positive constructs of youth wellness that contribute to resilient outcomes (Benard, 2004; WestEd, n.d.-b). Research has supported a strong relationship between resilience and SWB (Rodriguez-Fernandez et al., 2018), and this study validated the CHKS Core's ability to evaluate positive constructs related to SWB.

A method for accomplishing this aim was to establish the CHKS Core's concurrent validity in assessing student SWB. Concurrent validity is the "extent to which the results of a measure correlate with the results of an established measure of the same or a related underlying construct assessed within a similar time frame" (West \& Beckman, 2018, p. 2). Simply put, an adequate correlation within a similar measure verified to assess SWB would provide evidence of concurrent validity. Conversely, poor correlations would imply a lack of concurrent validity and, therefore, a limited or lack of ability to measure SWB using the CHKS Core.

In the evaluation of concurrent validity, prior studies have assessed the relationship between different measures using varied correlation statistics depending on the variables of analysis (Cullinan et al., 2002; DeSouza et al., 1994; Kovi et al., 2021; Peyton et al., 2020; Scattone et al., 2012; Sharp et al., 2010). The interpretation of correlational significance has been commonly established at $p<.01$ (DeSouza et al., 1994; Kovi et al., 2021; Peyton et al., 2020; Sharp et al., 2010). In one study evaluating the concurrent validity of the Scales for Assessing Emotional Disturbance (SAED), Cullinan et al. (2002) recommended selecting alpha of .001 to reduce the likelihood of falsely rejecting the null hypothesis of zero correlation. 
In addition to correlational significance, it is also common to interpret the magnitude or size of the correlation. To accomplish this, Cullinan et al. (2002) and others (DeSouza et al., 1994; Kovi et al., 2021; Peyton et al., 2020; Scattone et al., 2012; Sharpet et al., 2010) have recommended using Cohen's (1988) criteria, which defines values between .10 and .29 as small associations, values between .30 and .49 as medium associations, and values above .50 as large associations. Although it is common to use Cohen's criteria, other studies have used different guidelines. For example, in a study evaluating the concurrent validity of the 60 -second drawing test in measuring the closeness of high school students' relationships and symptoms of depression, it was suggested .30 rather than .50 could be interpreted as a large effect (Gignac \& Szodorai, 2016).

Given the different standards, various studies have argued a medium to large effect using Cohen's criteria (encompassing the range between .30 and .50 ) is sufficient to demonstrate concurrent validity. For example, Peyton et al. (2020) concluded concurrent validity existed between the Warner Initial Developmental Evaluation of Adaptive and Functional Skills (WIDEA-FS) and the Bayley Infant and Toddler Scales of Development based on a significant association between scales with effect sizes in the moderate to large range. Cullinan et al. (2002) interpreted concurrent validity similarly in their study of the SAED, which showed moderate to large correlations with other measures of emotional and behavioral problems. Overall, this is consistent with other studies that also have interpreted moderate to large correlations as representing evidence of concurrent validity (DeSouza et al., 1994; Kovi et al., 2021; Peyton et al., 2020; Scattone et al., 2012; Sharp et al., 2010). In addition to the interpretation of the strength of the correlation, the coefficient of determination, $R^{2}$, has been interpreted to evaluate the proportion variance (i.e., difference) between the two measures or variables assessed that is 
explainable by the each variable (Kovi, et al., 2021). Descriptively, in the evaluation of concurrent validity between two measures, $R^{2}$ provides for the percentage of variation between one measure that is explainable by the other measure. The range of this percentage is from $0 \%-$ $100 \%$. By Cohen's (1988) standard, 1\% reflects small variance, 9\% medium variance, and 25\% a large amount of variance that is explainable.

\section{Research Questions}

The purpose of the proposed study was to determine if the CHKS Core module demonstrated utility for assessing students using a dual continuum model of MH by evaluating indicators of SWB. When the CHKS Core showed this ability, it was then determined if a significant relationship existed between SWB and questions related to psychopathology on the CHKS Core survey module. The variables for analysis were defined as (a) the CHKS Core subscales and (b) SEHM Covitality Index.

There were two primary research questions in this study. Research Question 1 had six secondary questions that provided a focused analysis of the CHKS Core subscales related to the evaluation of those scales' ability to identify factors related to student SWB.

Research Question 1: Does the CHKS Core module gather information regarding SWB consistent with a bidimensional framework of $\mathrm{MH}$ ?

a. Does the caring adults in school scale of the CHKS Core denote a relationship with the total score on the SEHM Covitality Index?

b. Does the high expectations-adults scale of the CHKS Core denote a relationship with the total score on the SEHM Covitality Index?

c. Does the meaningful participation at school scale of the CHKS Core denote a relationship with the total score on the SEHM Covitality Index? 
d. Does the school connectedness scale of the CHKS Core denote a relationship with the total score on the SEHM Covitality Index?

e. Does the parent involvement scale of the CHKS Core denote a relationship with the total score on the SEHM Covitality Index?

f. Does the academic motivation scale of CHKS Core denote a relationship with the total score on the SEHM Covitality Index?

Research Question 2: Is there a relationship between psychopathology indicators on the CHKS Core and subscales correlated with SWB as identified in Research Question 1?

\section{Summary}

The promotion of $\mathrm{MH}$ wellness is essential to $\mathrm{MH}$ programing. Research has shown SWB to be a critical determinant (Antaramian et al., 2010; Greenspoon \& Salklofske, 2001). In schools, CHKS holds promise as a measure for evaluating youth SWB. Yet, as it stands, there has been a lack of research on the CHKS Core establishing it as a measure of SWB. Data from the 2016-2017 CHKS Core and SEHM survey modules were analyzed using Spearman's rankorder correlation to evaluate the relationship between (a) critical scales on the core survey hypothesized to assess aspects of SWB and (b) the SEHM Covitality Index. When a positive relationship was established, a point-biserial correlation was used to examine if a relationship existed between (a) the CHKS Core scales (RQ1 subset a-h) and (b) critical questions on the CHKS Core that evaluate aspects of psychopathology. The following chapter presents the literature on SWB, followed by a theoretical review of the CHKS Core and supplemental modules assessment of $\mathrm{MH}$ factors consistent with a bidimensional model of $\mathrm{MH}$. 


\section{CHAPTER 2: LITERATURE REVIEW}

As youth mental health $(\mathrm{MH})$ is best conceptualized through a bidimensional model that gives psychopathology and subjective well-being (SWB) equal importance in determining overall $\mathrm{MH}$, it is critical to review core features and empirical research of two leading bidimensional models. Accordingly, this chapter comprehensively presents reviewed literature on SWB as a construct critical to evaluating MH and the dual-factor model (DFM) and covitality model, the two leading frameworks in the bidimensional assessment of youth MH. Lastly, this chapter presents the California Healthy Kids Survey (CHKS) as a tool for assessing youth MH.

\section{Subjective Well-Being}

It is well established that the promotion of positive $\mathrm{MH}$ is a crucial component of a school-based mental health (SBMH) framework (Institute of Medicine, 2009). The World Health Organization (WHO) provided a definition of MH that fits this aim. The WHO (2013) characterized $\mathrm{MH}$ "as a state of well-being in which the individual realizes his or her abilities, can cope with the normal stresses of life, can work productively and fruitfully, and can make a contribution to his or her community" (p. 6). Historically, however, our understanding of MH has focused on the existence or absence of psychopathology (Ryff \& Singer, 1998), and positive MH was viewed merely as an absence of psychopathology. Through research in positive psychology, this idea has shifted gradually from this unidimensional framework to an approach that also considers positive aspects of $\mathrm{MH}$.

As far back as 1948, the WHO provided a definition of health that emphasized a state of well-being along with the absence of disease and infirmity. In the book, Current Concepts of Positive Mental Health, Jahoda (1958) argued the absence of psychopathology, or what they referred to as mental disease, was not sufficient for determining MH. Decades later, Ryff and Singer (1998) proposed the construct of MH should not simply involve the absence of 
psychopathology but also the existence of positive experiences (i.e., social contribution, life satisfaction, and self-acceptance).

Arising from the field of positive psychology, the construct of SWB focuses on the "how" and "why" of an individual's experience of their life. As Diener (1984) described, SWB refers, in its purest form, to how individuals experience their lives. The evaluation of SWB includes both cognitive and affective aspects, consisting of three main components: (a) positive affect, (b) negative affect, and (c) life satisfaction (Antaramian et al., 2010).

\section{Dual-Factor Model}

The DFM of MH, first explored by Greenspoon and Saklofske (2001) and later elaborated on by Suldo and Shaffer (2008), situates well-being as the interaction of two related factors: student psychopathology and SWB. Although the absence of psychopathology is one determinant of $\mathrm{MH}$, the model proposes SWB as a mediating factor in $\mathrm{MH}$ wellness. In the research literature, four distinct groups have emerged when both SWB and psychopathology are considered in determining MH. In a study of 407 elementary students, Greenspoon and Saklofske identified four distinct groups. These groups were later elaborated on by Antaramian et al. (2010) in a survey of middle school students to measure the MH status of youth. The first group represented students for whom SWB mediated the distress experienced by psychopathology and was labeled symptomatic but content. Conversely, youth who demonstrated no psychopathology but low SWB were described as vulnerable. Youth with high psychopathology and low SWB represented the group denoted as troubled, and those with low psychopathology and high SWB represented a group characterized as having complete positive MH. 
Subsequent studies have confirmed these four distinct groups' presence and the connected role of SWB to MH. In a study conducted by Lyons et al. (2013) evaluating 1,390 seventh- and 419 eighth-grade adolescents using both person- and variable-centered analysis, significant differences were found among the four groups of the DFM proposed by Greenspoon and Saklofske (2001). SWB was supported as a significant predictor beyond psychopathology for emotional, cognitive, and behavioral engagement. In another study by Renshaw and Cohen (2014), group differences were explored among the four-group model. In the study, 1,356 college undergraduates were placed in four distinct groups (i.e., mentally healthy, mentally unhealthy, symptomatic yet content, or asymptomatic yet discontent). Findings supported a bidimensional model of MH with measures of SWB (i.e., life satisfaction) appearing as a distinct indicator of functioning in academic, health, and social domains. Suldo et al. (2016) gathered data from 500 high school students and explored how the model relates to social and academic adjustment, and identifies development and physical health among adolescents. Findings of the study supported membership in the four distinct groups aligned with the dual continuum theory. Again, SWB showed significance as a determining variable in youth outcomes. Participants with complete MH (i.e., high SWB and low psychopathology) experienced more positive social and academic outcomes than those categorized as vulnerable (i.e., low SWB and low psychopathology).

A review of the proportion of class memberships in 1 of the 4 distinct groups generated by classifying $\mathrm{MH}$ functioning through a dual continua model demonstrated some consistency across studies. Those classified as experiencing complete MH (i.e., high SWB and low psychopathology) represented approximately $57 \%-67 \%$ of participants in the study's groups. Individuals representing the symptomatic but content profile (i.e., high SWB and high 
psychopathology) demonstrated a larger range at approximately $5 \%-17 \%$ of studied groups. Those falling in the vulnerable group (i.e., low SWB and low psychopathology) represented approximately $7 \%-18 \%$ of studied participants, and individuals showing membership in the troubled group (i.e., low SWB and high psychopathology) made up about 8\%-19\% of individuals when categorized into 1 of the 4 groups (see Table 2). Longitudinal analysis of this class membership's stability has been explored, with students experiencing complete $\mathrm{MH}$ attaining the highest class membership stability (Kelly et al., 2012).

\section{Table 2}

Dual Continua Model Class Membership

\begin{tabular}{lcccc}
\hline \multicolumn{1}{c}{ Article } & $\begin{array}{c}\text { Complete } \\
\text { MH/flourishing }\end{array}$ & $\begin{array}{c}\text { Symptomatic } \\
\text { but content }\end{array}$ & Vulnerable & Troubled \\
\hline Suldo et al., 2016 & $62 \%$ & $11.40 \%$ & $11.40 \%$ & $15 \%$ \\
Renshaw \& Cohen, 2014 & $61.40 \%$ & $4.80 \%$ & $18.70 \%$ & $19 \%$ \\
Lyons, 2013 & $64 \%$ & $8 \%$ & $7 \%$ & $19 \%$ \\
Antaramian et al., 2010 & $67 \%$ & $17 \%$ & $8 \%$ & $8 \%$ \\
Suldo \& Shaffer, 2008 & $57 \%$ & $13 \%$ & $13 \%$ & $17 \%$ \\
\hline
\end{tabular}

The relevance of SWB to school-related MH services has been well established. In a study of middle school students, Soldu and Schaffer (2008) found students with low psychopathology and high SWB had better achievement in reading, higher academic selfperceptions, fewer social problems and absences, and better peer and parent relationships than those who fell into the vulnerable category (i.e., low psychopathology and low SWB). In another study of middle school students, Antaramian et al. (2010) found students with complete MH had higher achievement and fewer behavioral problems than vulnerable students. Lyons et al. (2013) identified SWB as a primary predictor of GPA in middle school students along with emotional, cognitive, and behavioral engagement. Overall, students reporting higher SWB experienced more favorable academic and social outcomes. 
In summary, the empirical literature has underscored the importance of SWB as a critical factor to youth MH. It is appropriately derived from this model that viewing $\mathrm{MH}$ wellness as solely predicted by the presence or absence of psychopathology may lead to an over- or underestimation of student functioning. Although symptoms of psychopathology are a fair indicator of student risks for $\mathrm{MH}$ problems, SWB appears to function as either a mediator of psychopathology or an independent contributor to overall risk. As defined by this bidimensional MH model, the combined assessment of student $\mathrm{MH}$ wellness is valuable in ensuring accurate identification and treatment of student $\mathrm{MH}$ needs.

\section{Covitality}

The covitality model represents a bidimensional theory of MH where distress (i.e., psychopathology) and well-being are considered separate but related factors in the evaluation of MH wellness. The term covitality, first coined by Weiss and Enns (2002) in their study of the heritable nature of SWB, is defined as the "counterpart to comorbidity" (Furlong et al., 2014, p. 1013), with covitality conceptualized as the "synergistic effect of positive MH resulting from the interplay among multiple positive-psychological building blocks" (p. 1013).

Jones et al. (2013) conducted the first factor analysis to explore the concept of covitality as a unitary construct for the "synergy of positive psychology constructs and their relations with psychological well-being" (p. 512). In their study of college students, five factors were identified: (a) hedonia, (b) optimism, (c) self-efficacy, (d) hope, and (e) gratitude. These demonstrated to be first-order latent factors that loaded onto the second-order factor of covitality. The study suggested covitality was strongly related to well-being. Furlong et al. (2014) further established the validity and utility of the construct of adolescent covitality through confirmatory factor analysis (CFA), invariance analysis, and latent means testing of the Social-Emotional 
Health Module (SEHM), which was described as a "strength-based assessment linked to student mental health and well-being, academic success, and career and college readiness" (WestEd, n.d.-a, para. 9). Overall, path modeling demonstrated covitality was a strong predictor of students' SWB. Through the systematic examination of models best fitting the data, Furlong et al. (2014) established 12 core positive psychological constructs: (a) self-awareness, (b) selfefficacy, (c) persistence, (d) school support, (e) family coherence, (f) peer support, (g) emotion regulation, (h) empathy, (i) self-control, (j) optimism, (k) zest, and (l) gratitude. These constructs loaded onto the four latent factors of belief-in-self, belief-in-others, emotional competence, and engaged living. All constructs loaded onto the unifying factor of covitality and were positively correlated with SWB (i.e., Life Satisfaction, Positive and Negative Affect Scales for Children [PANAS-C]; see Figure 1).

\section{CHKS and Measurement of MH}

The CHKS has been purported to be a helpful guide to school districts and community stakeholders in planning prevention and intervention services for youth. It also holds promise for its application in youth MH (Austin \& O’Malley, 2012; WestEd, n.d.-a). Its application to youth MH has been derived from research that finds multiple correlations to student MH wellness measured in the CHKS Core and supplemental modules. Overall, the literature has supported MH as strongly connected to important school outcomes (Austin \& O’Malley, 2012), such as grades, attendance, and graduation (California Little Hoover Commission, 2001; Stoep et al., 2003). Factors such as school climate (La Salle et al., 2018; Shochet et al., 2006; Thapa et al., 2013 ), feelings of physical and social-emotional safety (La Salle et al., 2018), connectedness to peers and staff (La Salle et al., 2018; Shochet et al., 2006; Thapa et al., 2013), presence of supportive relationships (McNeely \& Falci, 2004; Waters \& Cross, 2010), high expectations 
(Ladd et al., 1999; Thapa et al., 2013), opportunities for meaningful participation and contribution (Ladd et al., 1999), and engagement in substance use (Austin \& O’Malley, 2012; Kandel et al., 1997; Substance Abuse and Mental Health Services Administration, 2011) are among some of the critical variables assessed concerning student $\mathrm{MH}$ and wellness in CHKS.

Other items assess indicators of psychopathology, including depression, stress, and anxiety (Austin \& O’Malley, 2012; Furlong et al., 2014).

\section{Figure 1}

Positive Mental Health and Covitality Model Underlying the SEHM

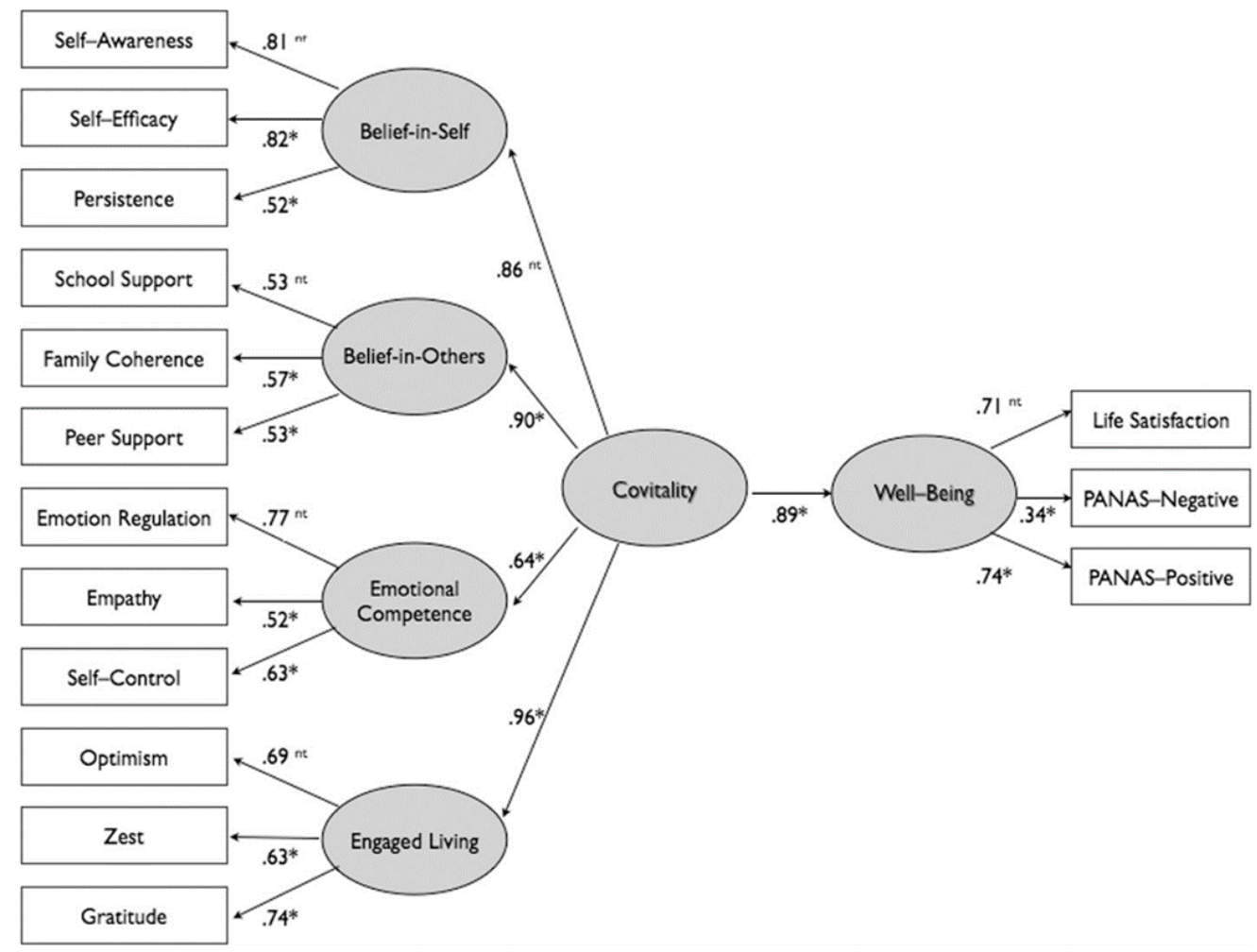

Note. Adapted from Preliminary Development and Validation of the Social and Emotional

Health Survey for Secondary Students, by M. Furlong et al., 2014, p. 1023. 
When comprehensively reviewed, the CHKS provides five broad areas contributing to the evaluation of student MH wellness (i.e., indicators of psychopathology, achievement and schoolrelated outcomes, substance abuse, school climate, and internal assets). The CHKS's comprehensive assessment of multiple indicators of health-related risk, behavior, and resilience/assets provides survey items that may appropriately fit this bidimensional model of MH. In reviewing this assessment, it is essential to look at those items indicating risk for psychopathology and those that show positive student assets and resilience related to SWB.

\section{Risk Indicators of Psychopathology}

\section{Direct Indicators of Psychopathology and MH}

The CHKS provides a focused evaluation of student $\mathrm{MH}$ wellness through questions assessing characteristics of depression, suicidal tendency, and anxiety. In a review of the CHKS, the high school core module provides three questions focused on evaluating feelings of depression and suicide (Questions 20c, 113, and 114) and an additional item surveying students' feelings of sadness, hopelessness, anxiety, stress, or anger (WestEd, n.d.-b). To complement these survey items and provide schools added specificity in identifying student needs, the SEHM has 56 additional questions allowing for a strength-based evaluation of student overall $\mathrm{MH}$ wellness (Questions 1-56; WestEd, n.d.-b). Developed to measure the "psychological building blocks of adolescent mental health" (Furlong et al., 2014, p. 1011), the SEHM works in alignment with the covitality model to assess belief-in-self, belief-in-others, emotional competence, and engaged living. This area provides a strong, connected relationship to measuring student indicators of psychopathology related to this bidimensional model of $\mathrm{MH}$ 
wellness. The CHKS expands this assessment by further measuring additional risk indicators related to psychopathology and student overall $\mathrm{MH}$ wellness.

\section{Achievement/School-Related Outcomes and MH}

Youth experiencing MH problems earn notably lower grades, have higher course failure rates, and demonstrate a higher occurrence of school dropout than other disability groups (Austin \& O’Malley, 2012). In a focused review of research, approximately $25 \%$ of students with emotional or behavioral health disorders graduate (California Little Hoover Commission, 2001). Overall, $46 \%$ of failures to complete school are associated with psychiatric disorders (Stoep et al., 2003). The CHKS importantly measures student achievement and other school-related outcomes on the core module. The core module provides four questions that hold relation to school achievement and related outcomes, one item surveying grades (Question 20), and three items (Questions 21-23) surveying school days missed (WestEd, n.d.-b). Four additional items assess perceived feelings of effort toward schoolwork (Items 33-36; WestEd, n.d.-b). Research has supported that $\mathrm{MH}$ problems in youth manifests in academic deficits compared to a typically developing peer (Austin \& O’Malley, 2012).

\section{Substance Abuse and $\mathrm{MH}$}

Although use of substances such as alcohol or drugs is not in and of itself an indicator of MH, substantial substance abuse may be an indicator or even become causal of MH problems (Austin \& O’Malley, 2012). The CHKS provides focused questions evaluating alcohol and drug use on core and alcohol and other drugs (AOD) modules. The core module offers 45 survey items (Questions 43-88), and the AOD module comprises 25 items focused on surveying students in the area (WestEd, n.d.-b). 
Research has underscored the association between substance use and MH disorders among youth populations (Austin \& O'Malley, 2012). Overall, there has been a high rate of cooccurrence between substance abuse and MH disorders in youth. Daily cigarette smoking, weekly alcohol consumption, and any illicit substance use in the past year are individually linked with the risk of diagnosable substance abuse and psychiatric disorders (i.e., anxiety, mood, or disruptive behavior disorders; Kandel et al., 1997). According to the 2010 National Survey on Drug Use and Health (Substance Abuse and Mental Health Services Administration, 2011), youths with a major depressive episode (MDE) were more likely than those without to engage in substance abuse (37.2\% vs. $17.8 \%$ ). In a review of the CHKS survey, Austin and O'Malley (2012) found youths' reported chronic sadness/hopelessness was associated with increased substance use compared to peers.

\section{Indicators of SWB (External and Internal Assets)}

\section{School Climate and MH}

School climate is a broad and multidimensional construct referring to multiple factors in the school environment associated with different student outcomes, including social-emotional and behavioral health (Salle et al., 2018). A well-studied concept, school climate, has varying definitions, yet critical features appear in each. The National School Climate Council (2007) recommended the following description: "School climate is based on patterns of people's experiences of school life and reflects norms, goals, values, interpersonal relationships, teaching and learning practices, and organizational structure" (p. 5). The standard features comprising school climate are peer and adult interpersonal relationships; instructional practices and 
expectations; organizational structures in school; and feelings of social, emotional, and physical safety (Appleton et al., 2008; Thapa et al., 2013).

The "CHKS reports include the percentage of students categorized as having high, medium, or low levels of school connectedness on a five-item scale derived from the National Longitudinal Study on Adolescent Health" (Austin \& O'Malley, 2012, p. 16). This construct represents a positive measure for evaluating students' perceived feelings of subjective wellness at school. The scale evaluates students' reported experience of closeness with people at their school, happiness to be at school, if they experience being a part of their school, and if they feel teachers treat them fairly. Key components measured on CHKS include the "presence of caring relationships, high expectation messages, and opportunities for meaningful participation, all of which enhance school connectedness, internal strengths (e.g., social skills), and positive academic and social-emotional student outcomes" (Austin \& O'Malley, 2012, p. 5).

\section{Connectedness and Supportive Relationships}

A key focus area of the CHKS is assessing the levels at which schools and communities provide students with factors that promote SWB. Austin and O'Malley (2012) wrote:

With three fundamental developmental supports that are linked to resilience and positive academic, social-emotional, and health outcomes even in the face of high-risk environmental conditions. I. caring, supportive adult relationships, II. messages that communicate high expectations for success, and III. opportunities for meaningful participation and contribution. Feelings of connectedness to peers, family, and adults in the school. (p. 48)

The CHKS Core and the California School Climate Survey (CSCS) survey provide multiple survey items assessing these areas. The core offers seven items that survey feelings of 
connection at school (Questions 24, 26, and 37-36). The CSCS provides a more comprehensive assessment of these factors with seven items (Questions 2, 3, and 6-10; WestEd, n.d.-b).

The CHKS, CSCS, and Resilience Youth Development Module (RYDM) further assess the level to which the home and peer environment provide these supports. The CSCS consists of five items that elicit responses about feelings of connection and support from peers at school (Questions 20-23 and 34). The RYDM has three items regarding supportive peer relationships (Questions 24-26), six items for supportive home (parent or adult) relationships (Questions 3035), and six items about out-of-home supportive adult relationships (Questions 39-44; WestEd, n.d.-b).

School "connectedness, academic achievement, positive school climates, and MH are all intertwined" (Austin \& O’Malley, 2013, p. 16). Overall, school connectedness is positively correlated to student achievement and wellness (La Salle et al., 2018; Shochet et al., 2006; Thapa et al., 2013). Although feelings of connectedness to teachers and families are studied less frequently, research has suggested these factors are related positively to student outcomes, such as achievement and MH wellness (Austin \& O’Malley, 2012). A comprehensive review of research on school climate conducted by Thapa et al. (2013) provided a supportive review that positive student-teacher relationships are strongly related to student behavioral and emotional engagement. Students' feelings of connectedness to families and peers were similarly positively related to student outcomes (McNeely \& Falci, 2004; Waters, 2008). Students’ perceived feelings of personal connection to their school are associated with lower levels of MH symptoms. In one study, feelings of school connectedness showed to be predictive of depressive symptoms, anxiety, and overall functioning in youth (Shochet et al., 2006). Peer relationships were also associated with decreased school conflict (Loukas et al., 2006). 


\section{Academic Rigor, Expectations, and Opportunities for Meaningful Participation}

Academic rigor, along with high expectations and opportunities for meaningful participation, are key constructs contributing to the school climate measured by CHKS. The CHKS Core, CSCS, and RYDM surveys provide multiple items evaluating these areas. In review, the core offers 14 items (Questions 22, 26, 30, and 37-47), which query students' feelings about rigor, expectations, and feelings of participation. The CSCS provides an additional 33 survey items (Questions 1-19, 24-27, 34, 41, 45-49, and 52-54) about academic rigor, expectations, and opportunities for participation. The RYDM provides an additional five questions (Items 30-35) evaluating the degree to which rigor and support are provided in the home (West Ed, n.d.-b).

The literature has shown an established connection between school-related expectations and supports as school climate elements and outcomes such as MH and wellness. Thapa et al. (2013) stated, "A positive school climate promotes cooperative learning, group cohesion, respect, and mutual trust" (p. 365). The research underscored when students are positively encouraged to participate in learning, their achievement scores increase (Ladd et al., 1999). Social-emotional outcomes are also related to school connectedness. One study of school connectedness identified student-perceived cohesion, friction, and overall satisfaction as indirectly linked with depressive symptoms and conduct problems (Loukas et al., 2006).

\section{Safety}

Maslow stated, "Feeling safe—socially, emotionally, intellectually, and physically—is a fundamental human need" (as cited in Thapa et al., 2013, p. 360). Accordingly, perceptions of safety and well-being are a well-established component of the school climate (Thapa et al., 2013). The CHKS comprehensively measures perceptions of safety in the core and CSCS 
modules. The core module represents 26 items related to feelings of safety in the school environment (Questions 22, 28, and 105-128). The CSCS provides 16 more survey items explicitly focused on school climate (Questions 23, 27-39, 47, and 50). A safety and violence module is also offered, providing a total of 14 items focused on surveying perceptions of safety and violence in the school and community, enhancing local education agencies' (LEAs) ability to assess youth risks and behavior (WestEd, n.d.-b).

Feelings of school safety include being afraid of bullying, getting into a fight, receiving bad grades, or being a victim of a crime (Lenzi et al., 2017). The literature has provided that feelings toward safety can be harmful to youth MH and can negatively affect academic attendance and academic engagement (Hughes et al., 2015; Lacoe, 2016; Nijs et al., 2011). Concerning student MH wellness, a study by the National Center for Education Statistics (1997) illustrated student feelings of safety were associated with improved psychological outcomes. Another study underscored perceptions of school safety as a protective factor in symptoms of depression (Ozer \& Weinstien, 2004). Further, Nijs et al.'s (2014) study on the perception of school safety and MH problems found perceptions of school safety were strongly and independently associated with self-reported MH problems in adolescents.

\section{Internal Assets and $\mathrm{MH}$}

Most connected to the evaluation of students' SWB may be their self-reported internal assets. Additional to the measurement of external risk or protective factors, the CHKS assesses internal student assets that bolster a student's resilience to $\mathrm{MH}$ problems and other risk outcomes. Expanding on the core module, the RYDM "assesses six personal social-emotional skills or resilience strengths: (a) communication and collaboration, (b) empathy, (c) self-efficacy, (d) self-awareness, (e) problem-solving, and (f) goals and aspirations" (WestEd, n.d.-a., para. 
29). The RYDM provides 23 questions (Questions 1-23) focused on assessing these internal student assets (i.e., resilience; WestEd, n.d.-b).

In the RYDM, resilience is described as "inborn developmental wisdom that naturally motivates individuals to meet their human needs for love, belonging, respect, identity, power, mastery, challenge, and meaning" (WestEd, 2002, as cited in Furlong et al., 2009, p. 36).

Research on resilience has provided that internal assets promote favorable social, emotional, and academic outcomes among youth (Benard, 2004). Research on CHKS has supported a relationship between these factors and student outcomes. Specifically, the presence of internal assets acts as a buffer to emotional distress for students exposed to bullying, a known correlate to the internalization of problems (Fredkove et al., 2019). In the covitality model, strengths and assets are components of overall covitality and are positive measures of overall student wellness and $\mathrm{MH}$.

\section{CHKS Theoretical Alignment With SWB}

In whole, as a comprehensive youth health and resilience survey, the CHKS's foundation is a well-supported theory of resilience (WestEd, n.d.a). Resilience is defined as "the capacity of a dynamic system to adapt successfully to disturbances that threaten system function, viability, or development" (Masten, 2015, p. 10) and is supported by both internal and external factors (Benard, 2004). Internal protective factors, described by Benard (2004) as personal strengths, are "individual characteristics, also called internal assets or personal competencies, associated with health develop and life success" (p. 13). External resilience, suitably described as environmental factors, are elements within the child's environment that serve to be protective against adversity and increase the likelihood of positive outcomes (Bernard, 2004). External factors present in the family, school, and community are supportive of external resilience (Benard, 2004). Components 
within these systems of support, identified consistently through the research and verified by Benard, are caring relationships, high expectations messages, and opportunities for participation and contribution.

Built off of Benard's (2004) theory of resilience, the CHKS encompasses a model that supports the evaluation of caring relationships, high expectations messages, and opportunities for participation and contribution (WestEd, n.d.-b). The CHKS Core uses this same terminology for scales evaluating these external factors (i.e., caring relationships, high expectations messages, and opportunities for participation and contribution). For the evaluation of both external and internal assets, the CHKS provides more items on the RYDM. Overall, research supports a strong relationship between resilience and SWB. Specifically, Rodriguez-Fernandez et al. (2018) identified resilience to exhibit predictive power for SWB. SWB was found to have a strong association with school engagement, which, in turn, was predictive of academic performance found to be predictive of perceived academic performance (see Figure 2).

\section{Figure 2}

Role of Resilience and Psychological Well-Being in School Engagement

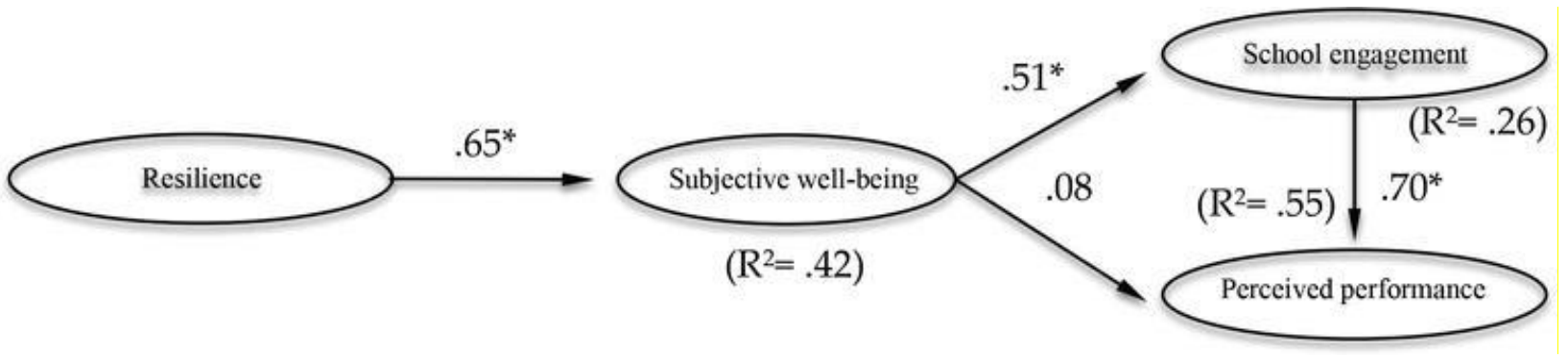




\section{Summary}

In summary, the CHKS gathers valuable information related to key indicators of student MH wellness that follow a bidimensional model of MH for indicators of psychopathology and SWB. As a survey suite, the CHKS provides information across critical areas such as feelings of physical and social-emotional safety, connectedness to peers and staff, presence of supportive relationships, high expectations, opportunities for meaningful participation and contribution, and engagement in substance use, which are among some of the critical variables assessed concerning student $\mathrm{MH}$ and wellness in the CHKS along with indicators of psychopathology. The CHKS Core provides an evaluation of valuable internal and external factors related to student $\mathrm{MH}$, which show alignment with SWB in the evaluation of external resilience factors for connectedness, presence of supportive relationships, high expectations, opportunities for meaningful participation and contribution. Other factors, such as engagement in substance use, may serve as additional indicators of psychopathology (Austin \& O’Malley, 2012; WestEd, n.d.a). Although the CHKS Core has demonstrated potential for evaluating student $\mathrm{MH}$ using a bidimensional model, no study to date has evaluated the core's measurement of student SWB, a critical component of a bidimensional model of mental health. This study addressed this critical gap. The following chapter outlines the study population, properties of the CHKS Core as the principal research measure, and analytic methods used to address the research questions postulated. 


\section{CHAPTER 3: RESEARCH METHODOLOGY}

In this chapter, the methodology of the study is outlined. First, the participant sample derived from the 2016-2017 California Healthy Kids Survey (CHKS) extant data set is discussed. Second, measures for the study are reviewed. Lastly, this chapter details the analysis of data as aligned with the outlined research questions.

\section{Participants}

The sample for analysis in this study was derived from the 2016-2017 extant CHKS survey data collected by WestEd through their collaboration with the California Department of Education (CDE) and a memorandum of understanding with Chapman University. Participants were drawn from both the 2016-2017 CHKS Core survey and the Social Emotional Health Survey (SEHM) for Grades 9-12 from participating California school districts. Approximately 85\% of California public schools administer CHKS to access Title IV funds (WestEd, n.d.-a). Descriptively, 346 school districts administered the 2016-2017 CHKS Core survey and 25 school districts administered the SEHM. A total of 308,635 students responded to questionnaires for Grades 9-12. To address missing data, listwise deletion was used to remove participants who did not respond to the items examined in the analyses. Lastly, a series of validity checks were conducted to respond to mischievous responders.

Mischievous responders are "youths who provide extreme, and potentially untruthful responses to multiple questions" (Robinson-Cimpian, 2014, p. 171). The prevalence of mischievous responders has been most notable among the adolescent population and is very common among self-administered questionnaires. This can be harmful to the validity of research data (Robinson-Cimpian, 2014). In this study, procedures for removing mischievous responders followed validity check criteria as Furlong et al. (2009) and Robinson-Cimpian (2014) 
recommended. Student data were run through a set of prescribed checks (see Figure 3). Students who violated two or more validity checks were removed from the data set for analysis.

\section{Figure 3}

Rejection Elements: Mischievous Responders

Inconsistent Responses 2 item pair, lifetime and current alcohol use

Item 47: Lifetime alcohol use (selected 0 use) and Item 59: 30-day alcohol use (selected 1 or more days used) $($ any inconsistency $=$ rejection $)$

Exaggerated Drug Use during the past 30 days how many days did you use . . . five or more drinks of alcohol in a row...

Item 59: 30 day 5 or more drinks in a row (20 or more days=rejected)

Use Nonexistent Drug Ever used fictitious drug, "Derbisol"

Item 50: Derbisol 1 (any response greater than $1=$ rejected)

Lack of honesty How many questions in this survey did you answer honestly

Item 106: How many questions answered honestly? (Only some and hardly any = rejected)

Total Score 0-1 = Valid

Total Score $\underline{2+=\text { Reject }}$

After removing missing data and performing analysis for rejection of mischievous responders, a total of 258,118 participants remained for analysis. Participant characteristics are summarized in Chapter 4.

\section{Measures: The CHKS}

For this study, the secondary CHKS Core and SEHM were analyzed. The CHKS is a comprehensive youth health and resilience survey employed statewide in California by the CDE through the nonprofit educational group, WestEd. The survey is required to be administered by 
local education agencies (LEAs) biennially to receive Title IV funds (WestEd, n.d.-a). The CHKS's purpose is to "provide data to guide efforts to reduce student health risk behaviors and build schools that are drug-free, safe and act as supportive havens for healthy positive youth development and achievement" (Austin \& Duerr, 2004, p. 1).

\section{Development}

As a survey measure, CHKS grew out of a desire to regularly collect representative statewide data of California secondary students' substance abuse habits in 1985. At that time, the Office of the Attorney General mandated evaluating the problem, leading to the development of the California Student Survey of Substance Use (CSS). As the survey continued through the California legislature, other agencies expanded its use through sponsorship. Starting in 1993, the Department of Alcohol and Drug Programs cosponsored the survey with the CDE. Later, the Department of Health Care Services and the CDE became the contracting agencies (Austin et al., 2018).

In time, the survey has grown to include questions about other health and risk behaviors, such as personal resilience, school climate, and safety. Because of district nonparticipation in the survey due to concerns driven from high stakes testing, the CSS merged into CHKS. At that time, the CDE contracted the nonprofit educational group, WestEd, to develop and administer the CSS and CHKS (Austin et al., 2018).

\section{Survey Content}

The CHKS focuses on evaluating all significant areas of health-related risk, behavior, and resilience (WestEd, n.d.-a). Developed as a survey to collect data for students in Grades 4-12, the CHKS consists of an elementary and secondary module. Both elementary and secondary survey suites include a core module and a set of supplemental modules that allow LEAs to 
customize their data collection based on areas of need for their unique population. For this study, the secondary CHKS Core survey and supplemental SEHM survey were used to analyze research questions for students in Grades 9-12.

\section{Core Module}

The secondary survey suite of CHKS consists of the core module and 16 specific supplemental modules. The core module used in this study is a self-response survey meant to support LEAs in their assessment of local control accountability plan requirements; students' attitudes, behaviors, and experiences related to school, academic, and social-emotional learning; positive development; and overall health and well-being (WestEd, n.d.-a). The 2016-2017 version of the core survey module consists of 118 questions for the high school module (see Appendix A). The questions focus on items considered most valuable for assessing school climate and safety, pupil engagement, student supports, bullying, substance abuse, and student MH for depression and suicidality (WestEd, n.d.-a). Additional demographic questions are surveyed to help identify critical subgroups (i.e., racial/ethnic groups, foster youth, economically disadvantaged youth, and English language learners). The secondary core is separated for middle and high school students, with the middle school survey consisting of shorter and simpler items that are developmentally appropriate for the group.

\section{Social-Emotional Health Module}

The secondary survey suite consists of 16 individual supplemental modules: (a) the school climate, (b) SEHM, (c) tobacco, (d) alcohol and other drugs (AOD), (e) building healthy communities, (f) Cal-well, (g) closing the achievement gap, (h) district after school, (i) drug-free communities, (j) gang risk awareness, (k) gender identity and sexual orientation-based harassment, (1) military-connected school, (m) physical health and nutrition, (n) resilience and 
youth development, (o) safety and violence, and (p) sexual behavior modules (WestEd, n.d.-a). The 2016-2017 secondary SEHM is the same for both middle and high school administration. The survey used in this study consisted of 46 survey items (see Appendix C). Developed and validated by Furlong et al. (2014), the survey was designed to "measure the psychological building blocks of adolescents' positive mental health" (p. 1011). In a study of students in eighth, 10th, and 12th grade, Furlong et al. established a theoretical model of covitality based on 12 indicators that contribute to four first-order factors (belief-in-self, belief-in-others, emotional competence, and engaged living). The analysis demonstrated 12 indicators of positive mental health load onto one meta-construct called covitality, a statistically validated predictor of students' subjective well-being (SWB; Furlong et al., 2014). Accordingly, the SEHM assesses youth in the areas of (a) empathy, (b) self-efficacy, (c) self-awareness, (d) persistence, (e) emotional self-regulation, (f) behavioral self-control, (g) gratitude, (h) zest, and (i) optimism.

\section{Reliability and Validity}

\section{Core Module}

Test developers at WestEd conducted a study in 2011 assessing the measurement analysis of specific questions on the CHKS Core and additional School Climate modules. To determine the measurement structure of focused items on the 2010-2011 surveys, the researchers completed a series of exploratory and confirmatory factor analyses (CFAs). The sample collected for the study was from the 2011 CHKS data reporting results from 294 high schools' ninth- and 10th- graders across a total of 58 school districts. The sample was heterogeneous by race and ethnicity (55\% Hispanic or Latino, 24\% White, 18\% Asian, 8\% African American, 52\% female, 48\% male, 51\% ninth-grade students, and 49\% 10th-grade students). 
The resulting analysis demonstrated the CHKS Core and School Climate modules showed good internal consistency. The analysis showed each measure represented a distinct dimension. Estimates were calculated using Cronbach's alpha coefficient, and, in general, 8 of 10 derived scales in the sample showed levels of reliability greater than 0.75 . For two exceptions (i.e., perceived safety and low ethnic tensions), reliability estimates were 0.52 to 0.59 , respectively. In their analysis (WestEd, 2011), models with the smallest number of factor loadings were selected over more complex models with cross loadings. After evaluating several models for cohesive effect, the resulting model generated seven factors in the Core module:

- $\quad$ school connectedness (4 items)

- $\quad$ support from adults in the school (6 items)

- opportunities for participation in meaningful activities (3 items)

- $\quad$ safety perceptions at school (1 item)

- $\quad$ low substance use (15 items)

- low nonphysical and physical violence victimization (11 items)

- low violence perpetration (7 items; WestEd, 2011)

\section{Social-Emotional Health Survey}

The secondary SEHM developed and validated by Furlong et al. (2014) and further verified by You et al. $(2014,2015)$ was designed to measure the metaconstruct of covitality. In their 2014 study on the development and validation of the SEHM for secondary school students, Furlong et al. conducted a series of CFAs, invariance testing, latent means testing, and pathmodeling analysis to assess the "psychological building blocks of adolescents' positive mental health" (p. 1011). The sample consisted of 4,189 students in Grades 8, 10, and 12 from 12 secondary schools (seven junior high schools, four comprehensive high schools, and one 
continuation high school). The sample was separated into two groups (subsample $1=2,056$ and subsample $2=2,133$ ) to support the statistical analysis of alternative models of youth $\mathrm{MH}$. The two subsamples' mean age was 15.1 , with a standard deviation of 1.7 and a range of $13-18$ years of age. The first subsample consisted of $73 \%$ of students who self-identified as Hispanic, and the other consisted of $71 \%$ who identified as Hispanic. The subsamples also had equal percentages of males and females (Furlong et al., 2014).

In their analysis, Furlong et al. (2014) evaluated the SEHM based on its construction consistent with an a priori theoretical model of 12 core positive-psychological building blocks drawn from the literature on youth-positive MH. Survey questions were derived from the Resilience and Youth Development Module (RYDM) part of the CHKS survey suite measuring youth's internal and external assets and other existing measures, which assessed different contributors to adolescents' positive MH (see Figure 4). To keep the survey parsimonious, researchers chose the three top-loading items from each established scale based on the results of factor analyses present in the research literature (Furlong et al., 2014). 


\section{Figure 4}

Theoretical and Measurement Model Underlying the SEHM

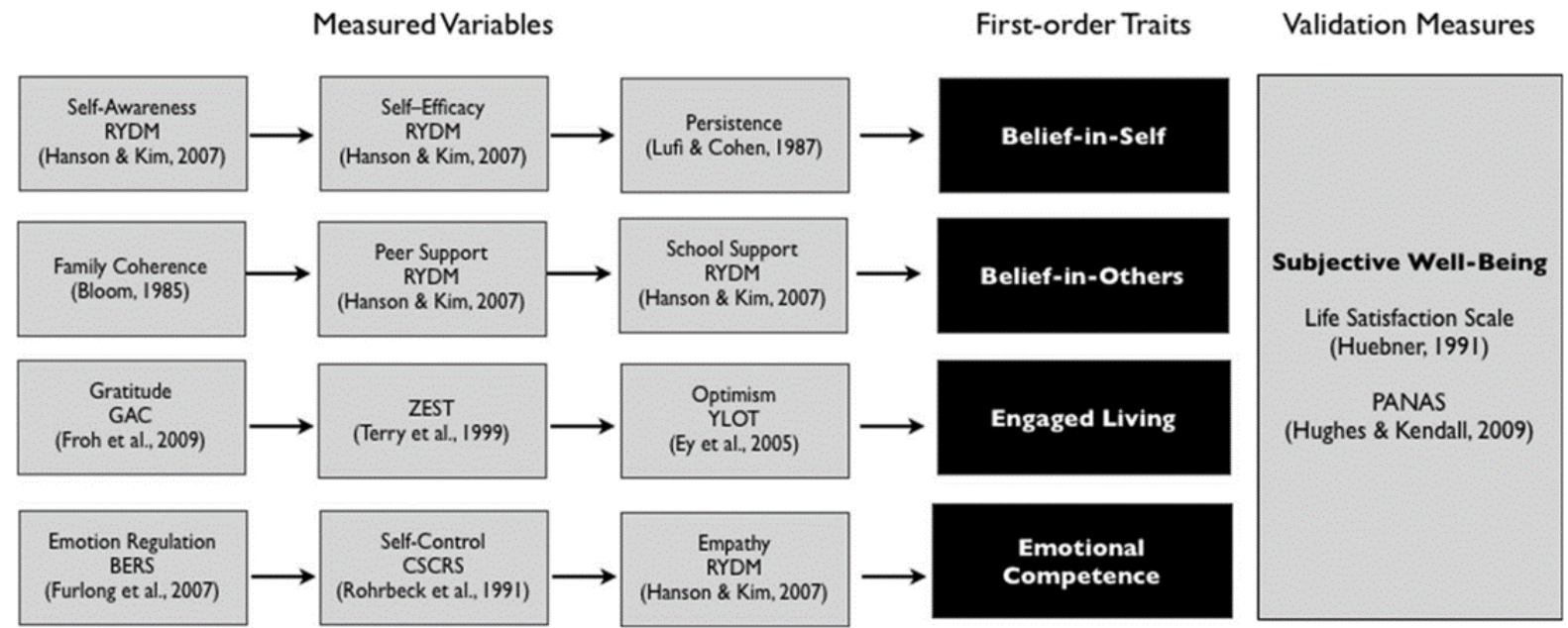

Note RYDM = Resilience Youth Developmental Module, GAC = Gratitude Adjective Checklist, YLOT $=$ Youth Life Orientation Test, BERS $=$ Behavioral Emotional Rating Scale (Student Version), CSCRS = Child Self-Control Rating Scale. Reprinted from Preliminary Development and Validation of the Social and Emotional Health Survey for Secondary Students, by M. Furlong et al., 2018, p. 1016.

Two measures, the Student Life Satisfaction Scale and Positive and Negative Affect Scales for Children (PANAS-C), were used to establish the factor structure of the SEHM as measures of SWB based on prior research on the dual continua model (Long et al., 2012; Park, 2004). The Student Life Satisfaction Scale was used to assess global life satisfaction, and the PANAS-C was used to assess recent emotional experiences within the last 2 weeks. Through CFA and structural equation modeling (SEM), Furlong et al. (2014) found positive and negative (reverse scored) indicators loading onto an SWB latent trait, with results indicating the model adequately fit the data. Results of the CFA and SEM showed factor loading on the hypothesized 12 constructs. After running several alternative factor models, Furlong et al. identified a 
preferred model solution that yielded four positive MH domains (i.e., belief-in-self, belief-in others, emotional competence, and engaged living). Invariance testing completed in three steps (configural, metric, and scalar) demonstrated the second-order covitality model had invariance across gender groups (male and female). Fit for genders male and female had established a statistically significant loading at the $p<.01$ level. Configural invariance and scalar invariance were additionally established. Path modeling, illustrated in the study, demonstrated significant positive relationships between the four positive $\mathrm{MH}$ domains (i.e., belief in self, belief in others, emotional competence, and engaged living) and adolescent covitality (see Figure 5). Covitality and SWB were established to have significant positive relationships (.89).

Finally, concurrent validity was established through one-way analysis of variance (ANOVA) and chi-square tests to support the adolescent covitality construct (Furlong et al., 2014). These analyses showed convergent validity for youth covitality levels (i.e., very low, low, high, and very high) and their perceptions of school safety and self-reported academic achievement. Discriminant validity was further established through findings showing higher covitality was inversely associated with substance use and depressive symptoms (Furlong et al., 2014). 


\section{Figure 5}

Positive Mental Health and Covitality Model Underlying the SEHM

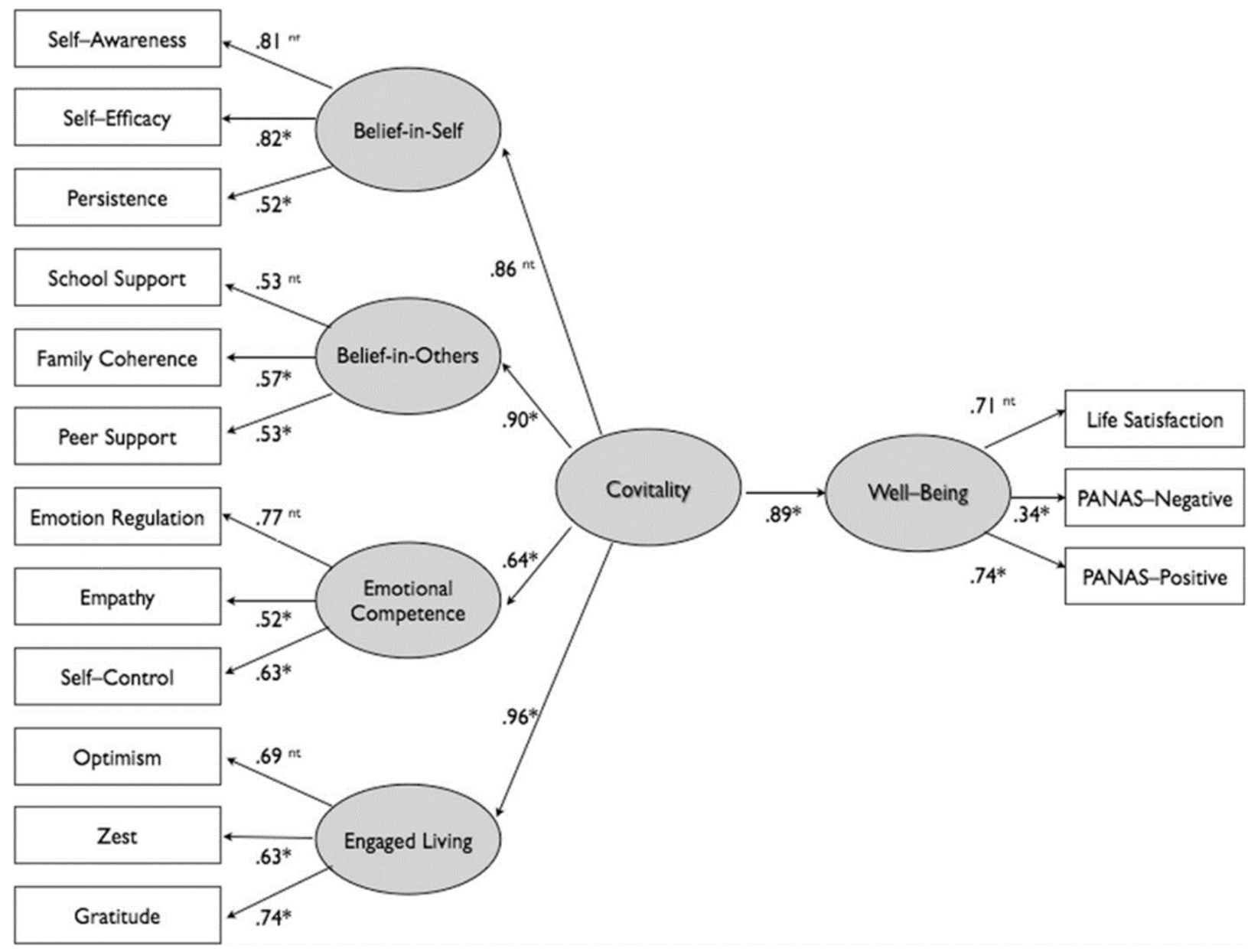

Note. Reprinted from Preliminary Development and Validation of the Social and Emotional Health Survey for Secondary Students, by M. Furlong et al., 2018, p. 1023.

Validation of the SEHM and the covitality construct for high school students was further established by You et al. (2014) and validated by You et al. (2015) for subcultural groups (i.e., Hispanic or Latino/a, White, blended, Black, Asian, Native Hawaiian/Pacific Islander, Alaskan/Native American). Specifically, You et al. (2014) established through CFA with a sample of 2,240 students in Grades 9-12 at two high schools (Latino/a heritage 72\%; 80\% at School 1 and $68 \%$ at School 2 experienced disadvantaged economic circumstances) the factor 
structure demonstrated by Furlong et al. (2014). Although latent means analysis found effect size differences, they were small to moderate for the belief-in-self and belief-in-others latent traits. SEM showed covitality to be a significant negative predictor of psychological distress (You et al., 2014).

Additionally, in a 2015 study, You et al. (2014) replicated the psychometric characteristics of the SEHM with a sample of 14,171 ethnically diverse high school students (Grades 9-12). Analysis of the CFA sample showed data fit the 12-factor model for covitality identified by Furlong et al. (2014). SEM and invariance testing across gender and sociocultural groups provided a model of full invariance across these groups (You et al., 2015).

\section{Analysis}

After considering sample size, the nature of the data, and the research questions, this study's questions appeared to be best answered by using descriptive and correlation statistics.

\section{Research Question 1}

Does the CHKS Core module gather information regarding SWB consistent with a bidimensional framework of MH?

a. Does the caring adults in school scale of the CHKS Core denote a relationship with the total score on the SEHM Covitality Index?

b. Does the high expectations-adults scale of the CHKS Core denote a relationship with the total score on the SEHM Covitality Index?

c. Does the meaningful participation at school scale of the CHKS Core denote a relationship with the total score on the SEHM Covitality Index?

d. Does the school connectedness scale of the CHKS Core denote a relationship with the total score on the SEHM Covitality Index? 
e. Does the parent involvement scale of the CHKS Core denote a relationship with the total score on the SEHM Covitality Index?

f. Does the academic motivation scale of the CHKS Core denote a relationship with the total score on the SEHM Covitality Index?

\section{Variables}

The two variables used to answer this research question were (a) the composite score of the identified scales on the CHKS Core for Q1 subsets "a" through "f" and (b) the total score on the SEHM Covitality Index.

\section{Analysis}

Given both variables are ordinal, and the question seeks a relationship between two variables, the Spearman rank-order correlation was best suited (Dellinger, 2017).

\section{Research Question 2}

Is there a relationship between psychopathology indicators on the CHKS Core and subscales correlated with SWB as identified in Research Question 1?

\section{Variables}

The variables for this research were (a) indicators of psychopathology as assessed by Question 20 Item C (During the past 30 days, did you miss school for any of the following reasons? C. Felt very sad, hopeless, anxious, stressed, or angry), Item 113 (During the past 12 months, did you ever feel so sad or hopeless almost every day for 2 weeks or more that you stopped doing some usual activities?) and Question 114 (During the past 12 months, did you ever seriously consider attempting suicide?) on the CHKS Core, and (b) the composite score of the identified scales on the CHKS Core for Q1 subsets "a" through "f." 


\section{Analysis}

The variables for this question represented one binary (i.e., nominal) variable defined by answers of "yes" or "no" to Questions 113 and 114 on the CHKS Core module and a set of continuous variables represented by the composite score generated for scales on the CHKS Core for Q1 subsets “a” through "f." With the intent to identify a relationship between a nominal and ordinal variable set, a Point-Biserial Correlation was used to determine the strength of the correlation (Chao, 2017).

\section{Spearman Rank-Order Correlation}

The Spearman rank-order correlation is a nonparametric statistical evaluation of the strength of the relationship among two variables. The analytical procedure is a variant of Pearson's product-moment correlation. Spearman's rank-order correlation is the best fit with ranked, ordinal data compared to the Pearson correlations, which are best suited for continuous variables (i.e., interval and ratio; Ellinger, 2017; Salkind, 2010).

\section{Assumptions}

Consistent with other correlation statistics, the rank order correlation evaluates the covarying relationship between sets of data (i.e., $x$ and y variables for each sample). With the computational focus of evaluating the relationship between ordinal variables, the statistical test requires a monotonic relationship (Salkind, 2010). Such a relationship asserts variables are arranged in ascending order and an increase in one variable may establish an increase in the other. Given the research questions posed in Q1 focused on seeking a positive directional relationship between the two variables to establish a measurable relationship with SWB, this assumption was met. 


\section{Interpretation}

The correlational statistic describes the strength of a relationship between the variables measured but does not establish whether the link is statistically different from 0 (Salkind, 2010). Given this, a test of statistical significance should be applied to determine whether the relationship exists and meets a level of significance for the sample set. For the Spearman rank order, it is recommended significance testing for large sample sizes be $z$ distribution compared to a $t$ distribution (Salkind, 2010). The larger the $z$ or $t$ value, the more probable it is the established relationship among variables is not random (i.e., error). Both statistics can be converted to a $p$ value to evaluate the probability of accepting or rejecting the null hypothesis (Dellinger, 2017). Salkind (2010) emphasized larger sample sizes combined with higher correlation coefficients (> .50 ), will yield $p$ values closer to 0 and suggest a statistically significant relationship. Given this, researchers must decide if the results are warranted or meaningful.

Also, researchers must be aware of the influence of tied rankings that can occur with ordinal data. When a high number of tied rankings are inherent in the data set, the research should statistically adjust with a correction factor to avoid inflation. It is worth noting that statistical computer packages (i.e., IBM SPSS) will automatically make these adjustments (Salkind, 2010).

\section{Point-Biserial Correlation}

The point-biserial correlation coefficient is a statistical procedure used to identify the relationship strength between two variables as paramount for other correlational statistics. The point-biserial method is correctly used when evaluating the relationship between a continuous variable (i.e., ratio or interval) and a dichotomous variable (i.e., ordinal; Chao, 2017). 


\section{Assumptions}

The point-biserial correlation holds three critical assumptions. First, it should have set as the independent variable dichotomous, nominal data. The other variable requires that it represents quantitative, interval, or ratio data. Secondly, the statistic calls for data to be normally distributed and will require more than 25 participants. Lastly, the statistic evaluates solely linear relationships (Chao, 2017). These assumptions were met by the research question posed for Q2, by which the two variables represent a dichotomous and quantitative data set. The ending sample for analysis exceeds the 25-student requirement at 258,118 students. A Kolmogorov-Smirnov test of normality and an evaluation of skewness and kurtosis indicated normality existed for the data set.

\section{Interpretation}

The interpretation of the point-biserial correlation is like what would be found with Pearson's $r$. Given this, the correlation statistic will be interpreted by both the strength (0 to 1.0) and the direction of the relationship (+ or -; Chao, 2017). Kemery et al. (1988) recommended a procedure for adjustment of the point-biserial correlations for "attenuation produced by inopportune splits in the dichotomous variable" (p. 688). This is derived from limitations inherent in analyzing dichotomous variables that present constrained variance when there are unequal proportions in the dichotomous categories. The procedure recommended to correct this is a conversion factor (Kemery et al., 1988). An estimation statistic for $r$ is produced to correct for the attenuation caused by unequal variables.

\section{Summary}

The proposed study's overall purpose was to enhance the understanding of the CHKS Core survey module's utility to assess student MH wellness consistent with the bidimensional 
model of youth MH by identifying the survey's potential to measure student SWB. Specifically, the study aimed to determine the CHKS Core's ability to measure SWB by establishing correlates of the measure with the SEHM Covitality Index, a validated determinant of student SWB (Furlong et al., 2014). Since the CHKS Core was validated for the assessment of SWB, the study further assessed the relationship between the CHKS Core scales correlated with SWB with indicators of psychopathology on the core. Evaluation of data for relationships between variables was achieved using the Pearson rank-order and point-biserial correlation statistics. 


\section{CHAPTER 4: RESULTS}

This study's focus was to analyze comprehensively the secondary California Healthy Kids Survey (CHKS) Core high school survey module to explore its ability to measure subjective well-being (SWB), a recognized critical component to the assessment of youth $\mathrm{MH}$ from a dual continuum perspective. This study also served to assess student outcomes along these same factors by using the CHKS Core module. In this chapter, the analysis of the research questions is presented. First, descriptive statics are provided for frequencies and percentages. Means and standard deviations were analyzed to explore trends in the interval-level variables. Next, to address the research questions, Spearman correlations and point-biserial correlations are reported. Statistical significance was evaluated at the generally accepted value, $\alpha=.05$.

\section{Frequencies and Percentages}

A total of 308,635 students responded to the questionnaire. Removals of mischievous responders brought the sample size down to 258,118 participants. For the correlational analyses to address the research questions, listwise deletion was used to remove participants who did not respond to the items examined in the analyses.

There was an approximate equal distribution of males $(n=123,302 ; 47.8 \%)$ and females $(n=127,039 ; 49.2 \%)$. Most of the sample was in ninth grade $(n=122,847 ; 47.6 \%)$ or 11 th grade $(n=106,887 ; 41.4 \%)$. A majority of the sample was of Hispanic or Latino origin $(n=$ $137,154 ; 53.1 \%)$. Most participants identified themselves as White $(n=78,204 ; 30.3 \%)$ or mixed race $(n=94,178 ; 36.5 \%)$. A majority of students indicated they were living in a home with one or more parents or guardians $(n=234,817 ; 91.0 \%)$. Many students indicated their parents graduated from college $(n=96,283 ; 37.3 \%)$, although nearly as many reported their parents graduated high school or less $(n=86,675 ; 33.6 \%)$. The sample was approximately even in distribution between students who received free or reduced lunches $(n=121,332 ; 47.0 \%)$ and 
students who did not receive free or reduced lunches $(n=106,168 ; 41.1 \%)$. English was the most often language spoken at home $(n=159,687 ; 61.9 \%)$. Most students identified their grades as mostly As and Bs $(n=84,354 ; 32.7 \%)$. A majority of students did not skip school during the previous 12 months at the time of the survey $(n=159,649 ; 61.5 \%)$. Frequencies and percentages for the demographic variables are presented in Table 3.

\section{Table 3}

Frequencies and Percentages for Demographic Variables

\begin{tabular}{|c|c|c|}
\hline Variable & $n$ & $\%$ \\
\hline \multicolumn{3}{|l|}{ Gender } \\
\hline Male & 123,302 & 47.8 \\
\hline Female & 127,039 & 49.2 \\
\hline No response & 7,777 & 3.0 \\
\hline \multicolumn{3}{|l|}{ Grade level } \\
\hline 9th grade & 122,847 & 47.6 \\
\hline 10th grade & 15,870 & 6.1 \\
\hline 11th grade & 106,887 & 41.4 \\
\hline 12th grade & 12,514 & 4.8 \\
\hline \multicolumn{3}{|l|}{ Are you Hispanic or Latino origin? } \\
\hline Yes & 137,154 & 53.1 \\
\hline No & 118,535 & 45.9 \\
\hline No response & 2,429 & 0.9 \\
\hline \multicolumn{3}{|l|}{ Race } \\
\hline White & 78,204 & 30.3 \\
\hline Black or African American & 11,238 & 4.4 \\
\hline Asian & 27,673 & 10.7 \\
\hline American Indian or Alaska Native & 9,478 & 3.7 \\
\hline Native Hawaiian or Pacific Islander & 4,557 & 1.8 \\
\hline Mixed (two or more) races & 94,178 & 36.5 \\
\hline No responses & 32,790 & 12.7 \\
\hline
\end{tabular}




\begin{tabular}{|c|c|c|}
\hline Variable & $n$ & $\%$ \\
\hline A home with one or more parents or guardians & 234,817 & 91.0 \\
\hline Other relative's home & 4,649 & 1.8 \\
\hline A home with more than one family & 9,155 & 3.5 \\
\hline Friend's home & 746 & 0.3 \\
\hline Foster home, group care, or waiting placement & 801 & 0.3 \\
\hline Hotel or motel & 360 & 0.1 \\
\hline Shelter, car, campground, or other transitional or temporary housing & 806 & 0.3 \\
\hline Other living arrangement & 5,415 & 2.1 \\
\hline No response & 1,369 & 0.5 \\
\hline \multicolumn{3}{|l|}{$\begin{array}{l}\text { What is the highest level of education with your parents or guardians } \\
\text { completed? }\end{array}$} \\
\hline Did not finish high school & 40,784 & 15.8 \\
\hline Graduated from high school & 45,891 & 17.8 \\
\hline Attended college but did not complete 4-year degree & 39,693 & 15.4 \\
\hline Graduated from college & 96,283 & 37.3 \\
\hline Don't know & 33,918 & 13.1 \\
\hline No response & 1,549 & 0.6 \\
\hline \multicolumn{3}{|l|}{ Do you receive free or reduced-price lunches at school? } \\
\hline Yes & 121,332 & 47.0 \\
\hline No & 106,168 & 41.1 \\
\hline Don't know & 24,171 & 9.4 \\
\hline No response & 6,447 & 2.5 \\
\hline \multicolumn{3}{|l|}{ What language is spoken most of the time in your home? } \\
\hline English & 159,687 & 61.9 \\
\hline Spanish & 74,759 & 29.0 \\
\hline Other & 22,639 & 0.9 \\
\hline No response & 1,033 & 0.4 \\
\hline \multicolumn{3}{|l|}{ In part 12 months, how would you describe the grades you received in school? } \\
\hline Mostly As & 47,697 & 18.5 \\
\hline As and $\mathrm{Bs}$ & 84,354 & 32.7 \\
\hline Mostly Bs & 22,743 & 8.8 \\
\hline Bs and Cs & 55,960 & 21.7 \\
\hline Mostly Cs & 14,705 & 5.7 \\
\hline
\end{tabular}




\begin{tabular}{lcc}
\hline Variable & $n$ & $\%$ \\
\hline Cs and Ds & 19,103 & 7.4 \\
Mostly Ds & 3,493 & 1.4 \\
Mostly Fs & 4,253 & 1.6 \\
No response & 5,810 & 2.3 \\
In the past 12 months, about how many times did you skip school or cut classes? & \\
0 times & 159,649 & 61.5 \\
1-2 times & 42,456 & 16.4 \\
A few times & 35,943 & 13.9 \\
Once a month & 5,894 & 2.3 \\
Once a week & 4,473 & 1.7 \\
More than once a week & 6,752 & 2.6 \\
No response & 3,951 & 1.5 \\
\hline
\end{tabular}

Note. Due to rounding errors, percentages may not equal $100 \%$.

A majority of the sample identified themselves as heterosexual $(n=203,224 ; 78.7 \%)$.

Frequencies and percentages for the sexual orientation among the participants are presented in Table 4.

The most prevalent reasons for students missing school were illness ( $n=127,479$; 49.4\%), not getting enough sleep $(n=40,335 ; 15.6 \%)$, feeling very sad, hopeless, anxious, stressed, or angry $(n=30,770 ; 11.9 \%)$, and being behind in schoolwork or not being prepared for a test or class assignment $(n=26,343 ; 10.2 \%)$. Frequencies and percentages for students' reasons for missing school during the previous 30 days are presented in Table 5. 


\section{Table 4}

Frequencies and Percentages for Sexual Orientation

\begin{tabular}{lcc}
\hline Variable & $n$ & $\%$ \\
\hline Heterosexual & & \\
Yes & 203,224 & 78.7 \\
No & 54,894 & 21.3 \\
Gay or lesbian & & \\
Yes & 7,530 & 2.9 \\
No & 250,588 & 97.1 \\
Bisexual & & \\
Yes & 18,766 & 7.3 \\
No & 239,352 & 92.7 \\
Transgender & & \\
Yes & 3,790 & 1.5 \\
No & 254,328 & 98.5 \\
Not sure & & \\
Yes & & \\
No & 239,766 & 92.9 \\
Decline to respond & & \\
Yes & 15,046 & 5.8 \\
No & 243,072 & 94.2 \\
\hline
\end{tabular}

Note. Due to rounding errors, percentages may not equal $100 \%$. 


\section{Table 5}

Frequencies and Percentages for Reason for Missing School in Previous 30 days

\begin{tabular}{|c|c|c|}
\hline Variable & $n$ & $\%$ \\
\hline \multicolumn{3}{|l|}{ Illness } \\
\hline Yes & 127,479 & 49.4 \\
\hline No & 130,639 & 50.6 \\
\hline \multicolumn{3}{|c|}{ Felt very sad, hopeless, anxious, stressed, or angry } \\
\hline Yes & 30,770 & 11.9 \\
\hline No & 227,348 & 88.1 \\
\hline \multicolumn{3}{|c|}{ Didn’t get enough sleep } \\
\hline Yes & 40,335 & 15.6 \\
\hline No & 217,783 & 84.4 \\
\hline \multicolumn{3}{|c|}{ Didn't feel safe at school } \\
\hline Yes & 5,272 & 2.0 \\
\hline No & 252,846 & 98.0 \\
\hline \multicolumn{3}{|c|}{ Had to work } \\
\hline Yes & 3,800 & 1.5 \\
\hline No & 254,318 & 98.5 \\
\hline \multicolumn{3}{|c|}{ Had to take care of or help a family member or friend } \\
\hline Yes & 16,497 & 6.4 \\
\hline No & 241,621 & 93.6 \\
\hline \multicolumn{3}{|c|}{ Wanted to spend time with friends who don't go to your school } \\
\hline Yes & 4,381 & 1.7 \\
\hline No & 253,737 & 98.3 \\
\hline \multicolumn{3}{|c|}{ Wanted to use alcohol or drugs } \\
\hline Yes & 3,559 & 1.4 \\
\hline No & 254,559 & 98.6 \\
\hline \multicolumn{3}{|c|}{ Were behind in schoolwork or weren't prepared for a test or class assignment } \\
\hline Yes & 26,343 & 10.2 \\
\hline No & 231,775 & 89.8 \\
\hline \multicolumn{3}{|c|}{ Were bored with or uninterested in school } \\
\hline Yes & 15,573 & 6.0 \\
\hline No & 242,545 & 94.0 \\
\hline \multicolumn{3}{|c|}{ Were suspended } \\
\hline Yes & 3,345 & 1.3 \\
\hline No & 254,773 & 98.7 \\
\hline
\end{tabular}




\begin{tabular}{lcc}
\hline Variable & $n$ & $\%$ \\
\hline Other reasons & & \\
Yes & 35,999 & 13.9 \\
No & 222,119 & 86.1 \\
\hline
\end{tabular}

Note. Due to rounding errors, percentages may not equal $100 \%$.

\section{Results for Research Question 1}

Research Question 1: Does the CHKS Core module gather information regarding SWB consistent with a bidimensional framework of $\mathrm{MH}$ ?

A series of Spearman correlations were used to explore the relationship between the CHKS Core subscales and the total score on the Social Emotional Health module (SEHM) Covitality Index. Cohen's standard (Cohen, 1988) was applied to determine the strength of the correlation coefficients. Coefficients between .10 and .29 represent a small association, values between .30 and .49 represent a medium association, and coefficients above .50 represent a large association.

Descriptive statistics used to evaluate trends of the CHKS Core subscales and the total score on the SEHM Covitality Index. The CHKS subscale with the largest mean score corresponded to academic motivation $(M=3.93, S D=0.85)$. The CHKS subscale with the lowest mean score corresponded to meaningful participation at school $(M=2.18, S D=0.85)$.

A series of Kolmogorov-Smirnov tests were performed to examine the normality assumption for the variables of interest. Every Kolmogorov-Smirnov test was not statistically significant $(p<.001)$, indicating the assumption of normality was not supported for the variables. Skewness and kurtosis also were used to examine how close the data resemble a normal distribution. According to Kline (2010), skewness and kurtosis within the range \pm 2.00 follow a normal distribution. The findings indicated the data fell within the threshold for a normal 
distribution. Also, Howell (2013) indicated data sets exceeding 50 cases tend to approximate normality. Descriptive statistics for the scales are presented in Table 6.

\section{Table 6}

Descriptive Statistics for the Scales

\begin{tabular}{lccccccc}
\hline Variable & $n$ & Min & Max & $M$ & $S D$ & Skewness & Kurtosis \\
\hline Caring adults in school & 254,356 & 1.00 & 4.00 & 2.73 & 0.83 & -0.18 & -0.76 \\
High expectations-adults & 254,320 & 1.00 & 4.00 & 2.97 & 0.83 & -0.51 & -0.53 \\
Meaningful participation at & & 1.00 & 4.00 & 2.18 & 0.85 & 0.45 & -0.62 \\
school & 254,751 & & & & & & \\
School connectedness & 252,611 & 1.00 & 5.00 & 3.57 & 0.82 & -0.64 & 0.60 \\
Parental involvement & 252,677 & 1.00 & 5.00 & 3.35 & 0.85 & -0.38 & 0.25 \\
Academic motivation & 252,688 & 1.00 & 5.00 & 3.93 & 0.85 & -0.97 & 1.35 \\
SEHM Covitality Index & 17,818 & 1.00 & 4.25 & 3.02 & 0.62 & -0.35 & 0.15 \\
\hline
\end{tabular}

Spearman correlations were performed to test the relationship between the CHKS Core subscales for Research Question 1, subquestions “a” to "f”" (see Table 7). All correlations were statistically significant, indicating as one subscale increased in value, the second also tended to increase. A majority of the relationships ranged from a moderate to a strong association.

A Spearman correlation was performed to investigate the two-way associations between caring adults in school and the SEHM Covitality Index. There was a significant association between caring adults in school and the SEHM Covitality Index, $r_{s}(17,805)=.51, p<.001$. The relationship was positive and represented a large effect, indicating as caring adults in school scores increased, the SEHM Covitality Index scores also increased. The coefficient of determination $-R^{2}$ - indicated approximately $26 \%$ of the variance in the SEHM Covitality Index could be explained by caring adults in school scores. Table 8 presents the Spearman correlation between the variables of interest. 


\section{Table 7}

Spearman Correlations Between the CHKS Core Subscales

\begin{tabular}{lcccccc}
\hline Variable & 1 & 2 & 3 & 4 & 5 & 6 \\
\hline 1. Caring adults in school & 1.00 & & & & & \\
2. High expectations-adults & $.82^{* *}$ & 1.00 & & & & \\
3. Meaningful participation at & $.47^{* *}$ & $.45^{* *}$ & 1.00 & & & \\
$\quad$ school & $.48^{* *}$ & $.47^{* *}$ & $.43^{* *}$ & 1.00 & & \\
4. School connectedness & $.37^{* *}$ & $.38^{* *}$ & $.29 * *$ & $.55^{* *}$ & 1.00 & \\
5. Parental involvement & $.32^{* *}$ & $.38^{* *}$ & $.36^{* *}$ & $.43^{* *}$ & $.36^{* *}$ & 1.00 \\
6. Academic motivation & & & & &
\end{tabular}

*** Denotes correlation is significant at .01 level.

\section{Table 8}

Spearman Correlation Between Caring Adults in School and SEHM Covitality Index

\begin{tabular}{lccc}
\hline Variable & \multicolumn{3}{c}{ SEHM covitality Index } \\
& $r_{s}(17,805)$ & $R^{2}$ & $p$ \\
\hline Caring adults in school & .51 & .26 & $<.001$ \\
\hline
\end{tabular}

A Spearman correlation was conducted to examine the two-way associations between high expectations-adults and SEHM Covitality Index. There was a significant association between high expectations-adults and SEHM Covitality Index, $r_{s}(17,815)=.53, p<.001$. The relationship was positive and represented a large effect, indicating as high expectations-adults scores increased, SEHM Covitality Index scores also increased. The coefficient of determination- $-R^{2}-$ indicated approximately $28 \%$ of the variance in the SEHM Covitality Index could be explained by high expectations-adults scores. Table 9 presents the Spearman correlation between the variables of interest. 


\section{Table 9}

Spearman Correlation Between High Expectations-Adults and SEHM Covitality Index

\begin{tabular}{lccc}
\hline Variable & \multicolumn{3}{c}{ SEHM Covitality Index } \\
& $r_{s}(17,815)$ & $R^{2}$ & $p$ \\
\hline High expectations-adults & .53 & .28 & $<.001$ \\
\hline
\end{tabular}

A Spearman correlation was conducted to examine the two-way associations between meaningful participation at school and SEHM Covitality Index. There was a significant association between meaningful participation at school and $\operatorname{SEHM~Covitality~} \operatorname{Index}, r_{s}(17,810)=$ $.48, p<.001$. The relationship was positive and represented a medium effect, indicating as meaningful participation at school scores increased, SEHM Covitality Index scores also increased. The coefficient of determination $-R^{2}$ - indicated approximately $23 \%$ of the variance in the SEHM Covitality Index could be explained by meaningful participation at school scores. Table 10 presents the Spearman correlation between the variables of interest.

\section{Table 10}

Spearman Correlation Between Meaningful Participation at School and SEHM Covitality Index

\begin{tabular}{lccc}
\hline Variable & \multicolumn{3}{c}{ SEHM Covitality Index } \\
& $r_{s}(17,810)$ & $R^{2}$ & $p$ \\
\hline Meaningful participation at school & .48 & .23 & $<.001$ \\
\hline
\end{tabular}

A Spearman correlation was conducted to examine the two-way associations between school connectedness and the SEHM Covitality Index. There was a significant association between school connectedness and the SEHM Covitality Index, $r_{s}(17,790)=.50, p<.001$. The relationship was positive and represented a large effect, indicating as school connectedness scores increased, SEHM covitality index scores also increased. The coefficient of determination $-R^{2}$ - indicated approximately $25 \%$ of the variance in the SEHM Covitality Index 
could be explained by school connectedness scores. Table 11 presents the Spearman correlation between the variables of interest.

\section{Table 11}

Spearman Correlation Between School Connectedness and SEHM Covitality Index

\begin{tabular}{lccc}
\hline Variable & \multicolumn{3}{c}{ SEHM Covitality Index } \\
& $r_{s}(17,990)$ & $R^{2}$ & $p$ \\
\hline School connectedness & .50 & .25 & $<.001$ \\
\hline
\end{tabular}

A Spearman correlation was conducted to examine the two-way associations between parental involvement and the SEHM Covitality Index. There was a significant association between parental involvement and the SEHM Covitality Index, $r_{s}(17,803)=.36, p<.001$. The relationship was positive and represented a moderate effect, indicating as parental involvement scores increased, SEHM Covitality Index scores also increased. The coefficient of determination $-R^{2}$ - indicated approximately $13 \%$ of the variance in SEHM Covitality Index could be explained by parental involvement scores. Table 12 presents the Spearman correlation between the variables of interest.

\section{Table 12}

Spearman Correlation Between Parental Involvement and SEHM Covitality Index

\begin{tabular}{lccc}
\hline Variable & \multicolumn{3}{c}{ SEHM Covitality Index } \\
& $r_{s}(17,803)$ & $R^{2}$ & $p$ \\
\hline Parental involvement & .36 & .13 & $<.001$ \\
\hline
\end{tabular}

A Spearman correlation was completed to assess the two-way associations between academic motivation and the SEHM Covitality Index. There was a significant association between academic motivation and the SEHM Covitality Index, $r_{s}(17,802)=.47, p<.001$. The 
relationship was positive and represented a moderate effect, indicating as academic motivation scores increased, SEHM Covitality Index scores also increased. The coefficient of determination $-R^{2}$ - indicated approximately $22 \%$ of the variance in the SEHM Covitality Index could be explained by academic motivation scores. Table 13 presents the Spearman correlation between the variables of interest.

\section{Table 13}

Spearman Correlation Between Academic Motivation and SEHM Covitality Index

\begin{tabular}{lccc}
\hline Variable & \multicolumn{3}{c}{ SEHM Covitality Index } \\
& $r_{s}(17,802)$ & $R^{2}$ & $p$ \\
\hline Academic motivation & .47 & .22 & $<.001$ \\
\hline
\end{tabular}

\section{Results for Research Question 2}

Research Question 2: Is there a relationship between indicators of psychopathology on the CHKS Core and subscales correlated with SWB as identified in Research Question 1?

A series of point-biserial correlations were completed to explore the relationship between indicators of psychopathology and the subscales on the CKHS Core. For the point-biserial correlations, the dichotomous variables were coded $1=$ yes and $0=n o$. Three individual items regarded as indicators of psychopathology were each examined separately. Cohen's standard (Cohen, 1988) was applied to determine the strength of the correlation coefficients. Coefficients between .10 and .29 represented a small association, values between .30 and .49 represented a medium association, and coefficients above .50 represented a large association. First, frequencies and percentages were examined for indicators of psychopathology (see Table 14). 


\section{Table 14}

Frequencies and Percentages for Indicators of Psychopathology

\begin{tabular}{lcc}
\hline Variable & $n$ & $\%$ \\
\hline Felt very sad, hopeless, anxious, stressed, or angry the previous 30 days & & \\
$\quad$ Yes & 30,770 & 11.9 \\
No & 227,348 & 88.1 \\
During the previous 12 months, did you ever feel so sad or hopeless almost & & \\
every day? & & \\
Yes & 77,244 & 29.9 \\
No & 175,708 & 68.1 \\
No response & 5,166 & 2.0 \\
During the previous 12 months, did you ever seriously consider attempting & & \\
suicide? & & \\
Yes & 38,666 & 15.0 \\
No & 212,665 & 82.4 \\
No response & 6,787 & 2.6 \\
\hline
\end{tabular}

Note. Due to rounding errors, percentages may not equal $100 \%$.

A point-biserial correlation was performed between feeling sad, hopeless, anxious, distressed, or angry during the previous 12 months, and the subscales correlated with SWB. The findings for all the point-biserial correlations were statistically significant $(p<.001)$. The correlation coefficients were all negative, suggesting students who responded they were sad, hopeless, anxious, distressed, or angry tended to have lower scores on the CHKS Core subscales. Applying Cohen's (1988) standard, it was determined each of the relationships represented a small effect. The coefficient of determination $-R^{2}$ - indicated $0-3 \%$ of the variance in the CHKS Core subscales could be explained by participants feeling sad, hopeless, anxious, distressed, or angry. Results of the point-biserial correlations are presented in Table 15. 


\section{Table 15}

Point-Biserial Correlation Between Very Sad, Hopeless, Anxious, Distressed, or Angry During Previous 12 Months and the CHKS Core Subscales

\begin{tabular}{lcccc}
\hline Variable & During the past 12 months, very sad, hopeless, anxious, distressed, or angry? \\
& $n$ & $r_{p b}$ & $R^{2}$ & $p$ \\
\hline Caring adults in school & 254,356 & -.07 & .00 & $<.001$ \\
$\begin{array}{l}\text { High expectations- } \\
\text { adults }\end{array}$ & 254,320 & -.09 & .01 & $<.001$ \\
$\begin{array}{l}\text { Meaningful } \\
\text { participation at school }\end{array}$ & 254,751 & & .01 & $<.001$ \\
School connectedness & 252,611 & -.08 & & \\
Parental involvement & 252,677 & -.16 & .03 & $<.001$ \\
Academic motivation & 252,688 & -.12 & .01 & $<.001$ \\
\hline
\end{tabular}

A point-biserial correlation was performed between feeling sad or hopeless almost every day for 2 weeks or more, and the subscales correlated with SWB. Findings for all the pointbiserial correlations were statistically significant $(p<.001)$. The correlation coefficients were all negative, demonstrating students who indicated they felt sad or hopeless almost every day for 2 weeks or more tended to have lower scores on the CHKS Core subscales. Applying Cohen's (1988) standard, it was determined each of the relationships represented a small effect. The coefficient of determination- $R^{2}$-indicated $1-5 \%$ of the variance in the CHKS Core subscales could be explained by participants feeling sad or hopeless almost every day for 2 weeks. Results of the point-biserial correlations are presented in Table 16. 


\section{Table 16}

Point-Biserial Correlation Between Feeling Sad or Hopeless Almost Every Day for 2 Weeks or More and the CHKS Core Subscales

\begin{tabular}{|c|c|c|c|c|}
\hline \multirow[t]{2}{*}{ Variable } & \multicolumn{4}{|c|}{$\begin{array}{c}\text { During the past } 12 \text { months, did you ever feel so sad or hopeless almost every } \\
\text { day for } 2 \text { weeks or more that you stopped doing some usual activities? }\end{array}$} \\
\hline & $n$ & $r_{p b}$ & $R^{2}$ & $p$ \\
\hline Caring adults in school & 249,777 & -.13 & .02 & $<.001$ \\
\hline $\begin{array}{l}\text { High expectations- } \\
\text { adults }\end{array}$ & 249,731 & -.13 & .02 & $<.001$ \\
\hline $\begin{array}{l}\text { Meaningful } \\
\text { participation at school }\end{array}$ & 250,158 & -.12 & .01 & $<.001$ \\
\hline School connectedness & 250,297 & -.23 & .05 & $<.001$ \\
\hline Parental involvement & 250,373 & -.14 & .02 & $<.001$ \\
\hline Academic motivation & 250,393 & -.11 & .01 & $<.001$ \\
\hline
\end{tabular}

A point-biserial correlation was conducted between considering attempting suicide, and the subscales correlated with SWB. Findings for all the point-biserial correlations were statistically significant $(p<.001)$. The correlation coefficients were all negative, indicating students who indicated they considered attempting suicide tended to have lower scores on the CHKS Core subscales. Applying Cohen's (1988) standard, it was determined each of the relationships represented a small effect. The coefficient of determination- $R^{2}-$ indicated $1 \%-$ $4 \%$ of the variance in the CHKS Core subscales could be explained by participants considering attempting suicide. Results of the point-biserial correlations are presented in Table 17. 


\section{Table 17}

Point-Biserial Correlation Between Considering Attempting Suicide During Previous 12 Months and the CHKS Core Subscales the CHKS Core

\begin{tabular}{lcccc}
\hline \multirow{2}{*}{ Variable } & \multicolumn{5}{c}{ During the past 12 months, did you ever seriously consider } \\
& $n$ & \multicolumn{5}{c}{ attempting suicide? } \\
& $n$ & $r_{p b}$ & $R^{2}$ & $p$ \\
\hline Caring adults in school & 250,413 & -.12 & .01 & $<.001$ \\
High expectations-adults & 250,368 & -.12 & .01 & $<.001$ \\
Meaningful participation at school & 248,529 & -.09 & .01 & $<.001$ \\
School connectedness & 248,672 & -.20 & .04 & $<.001$ \\
Parental involvement & 248,748 & -.13 & .02 & $<.001$ \\
Academic motivation & 248,759 & -.12 & .01 & $<.001$ \\
\hline
\end{tabular}

Frequencies and percentages for indicators of psychopathology were analyzed by a categorical break down of low, medium, and high for reported experiences of support across the following scales: (a) adults in the school, (b) high expectations-adults, (c) meaningful participation at school, (d) school connectedness, (e) parental involvement, and (f) academic motivation. Trends from the analysis indicate observable differences between students who experienced medium to high supports compared to those reporting low supports. Students who endorsed ratings of medium to high support across each category assessed (i.e., experienced support for caring adults in the school, high expectations-adults, meaningful participation at school, school connectedness, parental involvement, and academic motivation) evidenced an overall decrease in reported indicators of psychopathology. This trend was evident across all items for psychopathology. Frequencies and percentages for indicators of psychopathology by the categorical breakdown by scale are presented in Tables 18-23. 


\section{Table 18}

Frequencies and Percentages for Indicators of Psychopathology by Caring Adults in School

\begin{tabular}{lcccccc}
\hline Variable & \multicolumn{5}{c}{ Caring Adults in School } \\
\hline & Low & \multicolumn{2}{c}{ Medium } & \multicolumn{2}{c}{ High } \\
\hline & $n$ & $\%$ & $n$ & $\%$ & $n$ & $\%$ \\
\hline Felt very sad, hopeless, anxious, stressed, or angry & & & & & & \\
the previous 30 days & & & & & & \\
$\quad$ Yes & 6,730 & 18.2 & 15,646 & 11.1 & 8,016 & 10.5 \\
$\quad$ No & 30,307 & 81.8 & 125,181 & 88.9 & 68,476 & 89.5 \\
During the previous 12 months, did you ever feel & & & & & & \\
so sad or hopeless almost every day? & & & & & & \\
$\quad$ Yes & 16,178 & 43.7 & 41,008 & 29.1 & 19,251 & 25.2 \\
$\quad$ No & 20,216 & 54.6 & 97,519 & 69.2 & 55,605 & 72.7 \\
$\quad$ No response & 643 & 1.7 & 2,300 & 1.6 & 1,636 & 2.1 \\
During the previous 12 months, did you ever & & & & & & \\
seriously consider attempting suicide? & & & & & & \\
$\quad$ Yes & 9,500 & 25.7 & 19,915 & 14.1 & 9,127 & 11.9 \\
$\quad$ No & 26,984 & 72.9 & 118,973 & 84.5 & 65,914 & 86.2 \\
$\quad$ No response & 553 & 1.5 & 1,939 & 1.4 & 1,451 & 1.9 \\
\hline
\end{tabular}

Note. Due to rounding errors, percentages may not equal $100 \%$. 
Frequencies and percentages for indicators of psychopathology by the categorical breakdown of high expectations-adults are presented in Table 19.

\section{Table 19}

Frequencies and Percentages for Indicators of Psychopathology by High Expectations-Adults

\begin{tabular}{lcccccc}
\hline Variable & \multicolumn{5}{c}{ High Expectations-Adults } \\
\hline & $n$ & $\%$ & $n$ & $\%$ & $n$ & $\%$ \\
\hline & & & & & & \\
\hline Felt very sad, hopeless, anxious, stressed, or angry & & & & & & \\
the previous 30 days & 4,987 & 20.3 & 15,059 & 12.1 & 10,330 & 9.8 \\
$\quad$ Yes & 19,526 & 79.7 & 109,803 & 87.9 & 94,615 & 90.2 \\
$\quad$ No & & & & & & \\
During the previous 12 months, did you ever feel so & & & & & & \\
sad or hopeless almost every day? & 10,928 & 44.6 & 38,256 & 30.6 & 27,215 & 25.9 \\
$\quad$ Yes & 13,105 & 53.5 & 84,538 & 67.7 & 75,689 & 72.1 \\
$\quad$ No & 480 & 2.0 & 2,068 & 1.7 & 2,041 & 1.9 \\
$\quad$ No response & & & & & & \\
During the previous 12 months, did you ever & & & & & & \\
seriously consider attempting suicide? & 6,661 & 27.2 & 18,942 & 15.2 & 12,909 & 12.3 \\
$\quad$ Yes & 17,450 & 71.2 & 104,175 & 83.4 & 90,231 & 86.0 \\
$\quad$ No & 402 & 1.6 & 1,745 & 1.4 & 1,805 & 1.7 \\
$\quad$ No response & & & & & &
\end{tabular}

Note. Due to rounding errors, percentages may not equal $100 \%$. 
Frequencies and percentages for indicators of psychopathology by the categorical breakdown of meaningful participation at school are presented in Table 20.

\section{Table 20}

Frequencies and Percentages for Indicators of Psychopathology by Meaningful Participation at School

\begin{tabular}{lcccccc}
\hline Variable & \multicolumn{7}{c}{ Meaningful Participation at School } \\
\hline & \multicolumn{2}{c}{ Low } & \multicolumn{2}{c}{ Medium } & \multicolumn{2}{c}{ High } \\
\hline & $n$ & $\%$ & $n$ & $\%$ & $n$ & $\%$ \\
\hline Felt very sad, hopeless, anxious, stressed, or angry & & & & & & \\
the previous 30 days & & & & & & \\
$\quad$ Yes & 14,844 & 15.4 & 12,773 & 10.2 & 3,094 & 9.3 \\
$\quad$ No & 81,839 & 84.6 & 111,966 & 89.8 & 30,235 & 90.7 \\
During the previous 12 months, did you ever feel so & & & & & & \\
sad or hopeless almost every day? & & & & & & \\
$\quad$ Yes & 35,609 & 36.8 & 33,113 & 26.5 & 7,860 & 23.6 \\
$\quad$ No & 59,539 & 61.6 & 89,233 & 71.5 & 24,804 & 74.4 \\
$\quad$ No response & 1,535 & 1.6 & 2,393 & 1.9 & 665 & 2.0 \\
During the previous 12 months, did you ever & & & & & & \\
seriously consider attempting suicide? & & & & & & \\
$\quad$ Yes & 18,459 & 19.1 & 15,989 & 12.8 & 3,828 & 11.5 \\
$\quad$ No & 76,251 & 78.9 & 105,404 & 84.5 & 28,598 & 85.8 \\
$\quad$ No response & 1,973 & 2.0 & 3346 & 2.7 & 903 & 2.7 \\
\hline
\end{tabular}

Note. Due to rounding errors, percentages may not equal $100 \%$. 
Frequencies and percentages for indicators of psychopathology by the categorical breakdown of school connectedness are presented in Table 21.

\section{Table 21}

Frequencies and Percentages for Indicators of Psychopathology by School Connectedness

\begin{tabular}{lcccccc}
\hline Variable & \multicolumn{5}{c}{ School Connectedness } \\
\hline & \multicolumn{1}{c}{ Low } & \multicolumn{2}{c}{ Medium } & High \\
\hline & $n$ & $\%$ & $n$ & $\%$ & $n$ & $\%$ \\
\hline Felt very sad, hopeless, anxious, stressed, or angry & & & & & & \\
the previous 30 days & & & & & \\
$\quad$ Yes & 5,925 & 23.7 & 15,588 & 14.6 & 8,886 & 7.4 \\
$\quad$ No & 19,044 & 76.3 & 91,506 & 85.4 & 111,662 & 92.6 \\
During the previous 12 months, did you ever feel so & & & & & & \\
sad or hopeless almost every day? & & & & & & \\
$\quad$ Yes & 12,455 & 49.9 & 39,336 & 36.7 & 24,821 & 20.6 \\
$\quad$ No & 12,176 & 48.8 & 66,725 & 62.3 & 94,784 & 78.6 \\
$\quad$ No response & 338 & 1.4 & 1,033 & 1.0 & 943 & 0.8 \\
During the previous 12 months, did you ever & & & & & & \\
seriously consider attempting suicide? & & & & & & \\
$\quad$ Yes & 7,844 & 31.4 & 19,387 & 18.1 & 11,067 & 9.2 \\
$\quad$ No & 16,694 & 66.9 & 86,335 & 80.6 & 107,345 & 89.0 \\
$\quad$ No response & 431 & 1.7 & 1,372 & 1.3 & 2,136 & 1.8 \\
\hline
\end{tabular}

Note. Due to rounding errors, percentages may not equal $100 \%$. 
Frequencies and percentages for indicators of psychopathology by the categorical breakdown of parental involvement are presented in Table 22.

\section{Table 22}

Frequencies and Percentages for Indicators of Psychopathology by Parental Involvement

\begin{tabular}{lcccccc}
\hline Variable & \multicolumn{5}{c}{ Parental Involvement } \\
\hline & \multicolumn{2}{c}{ Low } & \multicolumn{2}{c}{ Medium } & \multicolumn{2}{c}{ High } \\
\hline & $n$ & $\%$ & $n$ & $\%$ & $n$ & $\%$ \\
\hline Felt very sad, hopeless, anxious, stressed, or angry & & & & & & \\
the previous 30 days & & & & & & \\
$\quad$ Yes & 10,616 & 18.5 & 13,897 & 11.6 & 5,901 & 7.8 \\
$\quad$ No & 46,852 & 81.5 & 105,579 & 88.4 & 69,832 & 92.2 \\
During the previous 12 months, did you ever feel so & & & & & & \\
sad or hopeless almost every day? & & & & & & \\
$\quad$ Yes & 23,080 & 40.2 & 36,643 & 30.7 & 16,905 & 22.3 \\
$\quad$ No & 33,765 & 58.2 & 81,766 & 68.4 & 58,214 & 76.9 \\
$\quad$ No response & 623 & 1.1 & 1,067 & 0.9 & 614 & 0.8 \\
During the previous 12 months, did you ever & & & & & & \\
seriously consider attempting suicide? & & & & & & \\
$\quad$ Yes & 12,858 & 22.4 & 17,725 & 14.8 & 7,723 & 10.2 \\
$\quad$ No & 43,702 & 76.0 & 99,998 & 83.7 & 66,742 & 88.1 \\
$\quad$ No response & 908 & 1.6 & 1753 & 1.5 & 1,268 & 1.7 \\
\hline
\end{tabular}

Note. Due to rounding errors, percentages may not equal $100 \%$. 
Frequencies and percentages for indicators of psychopathology by the categorical breakdown of academic motivation are presented in Table 23.

\section{Table 23}

Frequencies and Percentages for Indicators of Psychopathology by Academic Motivation

\begin{tabular}{lcccccc}
\hline Variable & \multicolumn{5}{c}{ Academic Motivation } \\
\hline & $n$ & $\%$ & $n$ & $\%$ & $n$ & $\%$ \\
\hline & & & & & & \\
\hline Felt very sad, hopeless, anxious, stressed, or angry & & & & & & \\
the previous 30 days & 9,848 & 17.8 & 14,031 & 11.8 & 6,526 & 8.3 \\
$\quad$ Yes & 45,432 & 82.2 & 104,731 & 88.2 & 72,170 & 91.7 \\
$\quad$ No & & & & & & \\
During the previous 12 months, did you ever feel so & & & & & & \\
sad or hopeless almost every day? & & & & & \\
$\quad$ Yes & 21,107 & 38.2 & 35,767 & 30.1 & 19,755 & 25.1 \\
$\quad$ No & 33,504 & 60.6 & 81,960 & 69.0 & 58,300 & 74.1 \\
$\quad$ No response & 669 & 1.2 & 1,035 & 0.9 & 591 & 0.8 \\
During the previous 12 months, did you ever & & & & & & \\
seriously consider attempting suicide? & & & & & & \\
$\quad$ Yes & 12,278 & 22.2 & 17,006 & 14.3 & 9,021 & 11.5 \\
$\quad$ No & 41,960 & 75.9 & 100,171 & 84.3 & 68,323 & 86.9 \\
$\quad$ No response & 1,042 & 1.9 & 1,585 & 1.3 & 1,302 & 1.7 \\
\hline
\end{tabular}

Note. Due to rounding errors, percentages may not equal $100 \%$. 


\section{Summary}

This study comprehensively analyzed the secondary CHKS Core survey module to explore its ability to measure SWB, a component of the evaluation of $\mathrm{MH}$ in the dual continuum theory. This study also expanded on the research by assessing student outcomes along these same factors by using the CHKS Core module. In this chapter, data were analyzed and presented. Frequencies and percentages were used to explore trends in the nominal variables. Means and standard deviations were used to investigate any trends in the interval-level variables.

To address the study's research questions, Spearman correlations and point-biserial correlations were conducted. The resulting analysis showed significant, positive relationships between each of the CHKS Core subscales hypothesized as having a relationship with SWB and the SEHM Covitality Index. Also, there were significant, inverse relationships between the CHKS Core subscales and indicators of psychopathology. Statistical significance was evaluated at the generally accepted value, $\alpha=.05$. Frequencies and percentages were presented for the CHKS Core subscales and indicators of psychopathology. In the next chapter, the implications of these findings are discussed in the context of current literature. Limitations of this research and recommendations for future studies are also addressed. 


\section{CHAPTER 5: DISCUSSION}

In this study, the extant data from the 2016-2017 California Healthy Kids Survey (CHKS) high school core and the Social-Emotional Health Module (SEHM) was analyzed to explore the ability of the CHKS to measure subjective well-being (SWB). SWB is a critical component to the assessment of youth mental health $(\mathrm{MH})$ consistent with the dual continuum theory. The CHKS Core module is the most widely used survey among the CHKS survey suite (WestEd, n.d.-a). Given this and the absence of research exploring the CHKS Core's utility in evaluating student $\mathrm{MH}$, a critical gap in the literature was apparent. This gap was attenuated by analyzing the CHKS high school core scales, which evidenced conceptual alignment with SWB against the SEHM Covitality Index, an established measure of youth SWB (Furlong et al., 2014; You et al., 2015). The implications to stakeholders in school-based MH are considerable. In this chapter, findings from the analysis are discussed as related to the research questions. Implications of the results are also explored.

\section{Discussion of Research Questions}

Research Question 1: Does the CHKS Core module gather information regarding subjective well-being consistent with a bidimensional framework of MH?

Research Question 2: Is there a relationship between psychopathology indicators on the CHKS Core and subscales correlated with SWB as identified in Research Question 1?

This study's primary research question focused on evaluating the CHKS Core and its ability to assess student SWB. Through correlational analysis of six scales on the CHKS high school core with the SEHM Covitality Index, this study established concurrent validity with the SEHM Covitality Index. All six scales on the CHKS Core (i.e., caring adults in school, high expectations, meaningful participation, school connectedness, parent involvement, and academic motivation) evidenced a significant positive relationship with the SEHM Covitality index as an 
established measure of student SWB. Therefore, as students rated themselves higher across the six areas on the core, their traits for SWB also went up as measured by the SEHM Covitality Index. Each scale demonstrated significance at the accepted value, $\alpha=.05$.

Although some variance was observed in the strength of the relationship, all scales showed a moderate to large association with covitality (Cohen, 1988). Establishment of this relationship between each scale and covitality is further supported through considering the implications beyond statistical significance with the model fit assessed by the coefficient of determination, $R^{2}$. Specifically, the amount of variance in the SEHM Covitality Index accounted for by the CHKS Core scales showed consistency across analyzed scales. Overall, 22\%-28\% of the variance in covitality was explained by the analyzed core scales. The exception to this was parental involvement, in which only $13 \%$ of the variance was explainable. Prior research has supported the establishment of concurrent validity at a medium to large effect size by Cohen's (1988) standard in the interpretation of both the correlation coefficient and $R^{2}$ (DeSouza et al., 1994; Kovi et al., 2021; Peyton et al., 2020; Scattone et al., 2012; Sharp et al., 2010). The results are therefore supportive of the hypothesis that the secondary CHKS Core survey module can measure SWB, a critical component of youth $\mathrm{MH}$ assessment consistent with the dual continuum theory.

In this study, the secondary research question was: Should the CHKS Core show concurrent validity in the assessment of SWB to determine if a significant relationship then exists between SWB and items related to psychopathology on the CHKS Core survey module? This question held significance to stakeholders in school-based mental health (SBMH). The first had to do with developing a conceptual alignment with the CHKS data to a bidimensional model of $\mathrm{MH}$. With research demonstrating SWB and psychopathology coexist on a continuum of $\mathrm{MH}$ 
(Greenspoon \& Saklofske, 2001; Suldo \& Shaffer, 2008), it would be expected any scale that is a proxy to SWB would demonstrate an inverse relationship with measures of psychopathology. For stakeholders in SBMH, the relationship among the variables is important for system-level evaluation and program planning. Identification of students by needs in these two critical areas (i.e., SWB and psychopathology) may allow for local education agencies (LEAs) and other stakeholders to focus resources to those selected areas with greater specificity.

In this study, a series of point-biserial correlations were performed to explore the relationship between indicators of psychopathology and subscales on the CKHS Core. For this, the analysis focused on three survey questions from the CHKS Core that are indicators of psychopathology. The questions surveyed students for experiences of sadness, hopelessness, anxiousness, stress, anger, and suicidality. These characteristics relate to depression and anxiety, which are among some of the highest occurring MH problems among youth (Centers for Disease Control and Prevention, 2013). The importance of screening for suicide is equally qualified given it ranks as the second leading cause of death for all youth (Centers for Disease Control and Prevention, 2013). Results of the analysis showed significance $(p<.001)$ across all six scales evaluated (i.e., caring adults in school, high expectations, meaningful participation, school connectedness, parent involvement, and academic motivation). The relationship was negative, meaning students who endorsed feelings of psychopathology tended to have lower scores on the CHKS Core subscales correlated with SWB.

This relationship is consistent with expectations and aligns with the literature (Antaramian et al., 2010; Diener, 2013; Greenspoon \& Saklofske, 2001; Kelly, 2012; Lyons et al., 2013; Renshaw \& Cohen, 2013). Although other studies have reported a stronger inverse relationship between psychopathology and SWB, what is notable is that an inverse relationship 
between psychopathology and SWB remained evident even given the limited number of items addressing psychopathology. Other studies have used robust psychometrically validated measures with more items (Antaramian et al., 2010; Diener, 2013; Greenspoon \& Saklofske, 2001). The results may also support the independence of psychopathology from SWB, which supports the theory that high SWB can serve as a mitigating factor to distress. However, the results must be interpreted with caution, given that the results demonstrate a small effect by Cohen's (1988) standard, and only 0-5\% of the variance in the CHKS Core subscales could be explained by participant responses to these questions.

Given this, the meaning in the relationship between the variables is best analyzed through descriptive statistics. This analysis showed that students' reports of psychopathology changed based on their perceptions of school-related support associated with SWB. Notably, students who reported medium to high supports reported considerably fewer indicators of psychopathology than those who rated themselves low for school support, an important indicator of SWB. The following section discusses the results for both research questions by each of the six scales found significant in correlation with covitality on the SEHM and holding some relationship with psychopathology.

\section{Caring Adults in School}

The caring adults scale on the CHKS Core consisted of three questions, which evaluate the degree students experience or perceive an adult at school caring about them. Consistent with prior studies, the study identified a positive relationship between students' experience of a positive relationship at school and other positive outcomes such as achievement, MH wellness, and behavioral and emotional engagement (La Salle et al., 2018; Loukas et al., 2005; Shochet et 
al., 2006; Thapa et al., 2013). In the study, the relationship was established with SWB by correlation with the SEHM Covitality Index.

Other studies have found overall positive student-teacher relationships are related to student behavioral and emotional engagement (Akey, 2006; Thapa et al., 2013). Other studies looking at the psychometric properties of scales and model fit to positive student well-being show caring adult relationships to have both a theoretical and statical pathway to SWB. Specifically, in their analysis of the psychometric properties of the CHKS Youth and Development Module, Hanson and Kim (2007) identified school support (i.e., caring adult relationships, high expectations, and meaningful participation) to contribute to factors of resilience, which were associated with improved outcomes for youth. Furlong et al. (2014), in their study to establish the SEHM and identify the construct covitality - a strong correlate to SWB — found model fit with school support consistent with Hansen \& Kim (2007). Overall, this finding provides support for the CHKS Core's potential as a measure of SWB, with the caring relationship scale showing contribution as one factor to this measurement among the other CHKS scales given the evidence of a large effect $\left(r_{s}=.51\right)$ and $26 \%$ of variance accountable. This level of significance is consistent with other studies that have established concurrent validity at a moderate to large effect per Cohen's (1988) standard (DeSouza et al., 1994; Kovi et al., 2021; Peyton et al., 2020; Scattone et al., 2012; Sharp et al., 2010).

\section{Indicators of Psychopathology by Caring Adults in School}

Students experiencing different levels of support (low, medium, or high) from caring adults at school reported differences in their experiences of feeling sad, hopeless, anxious, stressed, or angry in the past 30 days. Descriptively, for students who rated themselves as low in experiencing caring adult relationships in school, $18.2 \%(n=6,730)$ reported feeling sad, 
hopeless, anxious, stressed, or angry, compared to $11.1 \%(n=15,646)$ experiencing medium levels of caring adult relationships and $10.5 \%(n=8,016)$ experiencing high caring adult relationships. Although the differences do not meet a high threshold for establishing a moderate to large effect (Cohen, 1988), the data hold relevance for LEAs and other stakeholders seeking to understand the MH needs of their student population.

Similar results were observed for students reporting they felt sad or hopeless almost every day for the last 12 months. For these students, the experience of caring adult relationships at the low, medium, or high level showed bearing on their report of these symptoms of psychopathology. Specifically, $43.7 \%(n=16,178)$ of students low in their experience of caring adults reported they experienced these feelings compared to $29.1 \%(n=41,008)$ who experienced medium levels of caring adult relationships and 25.2\% $(n=19,251)$ who reported high levels.

Results extend in a similar manner for student reports of suicidality during the last 12 months. For students experiencing low levels of adult caring relationships, $25.7 \%(n=9,500)$ of students reported they have seriously considered attempting suicide, compared to $14.1 \%$ ( $n=$ $19,915)$ and $11.9 \%(n=9,127)$ in the medium and high support categories, respectively.

Importance of these results can then be drawn from their alignment with the literature showing caring adult relationships are a significant school-level support that contributes to student resilience and improved outcomes (Hanson \& Kim, 2007) and holds a relationship to student SWB (Furlong et al., 2014). Moreover, importance is drawn from the weight of the reported experiences. Students experiencing symptoms of depression, anxiety, and reported contemplation of suicide require instrumental support. For LEAs and other stakeholders in 
SBMH, the use of this data supports the identification of missing school-based supports that may mitigate distress, as identified by indicators of psychopathology.

\section{High Expectations-Adults}

The level high expectations, as communicated by encouragement, is measured by three questions on the CHKS Core survey. Research by Hanson and Kim (2007) suggested high expectations from adults to relate to positive student outcomes as a school support, much like caring adult relationships. Furlong et al. (2014) established a link between school supports (i.e., caring adult relationships, high expectations, and meaningful participation) and SWB. Within this study, the high expectations scale was explored as a correlate to SWB. Like the caring adults scale, it evidenced a large effect, showing a significant association between high expectationsadults and the SEHM Covitality Index, with $28 \%$ of the variance in the covitality index explained by high expectations as measured on the CHKS Core. This level of significance is comparative to prior studies that established concurrent validity at a moderate to large effect or percentage of accountable variance per Cohen's (1988) standard (DeSouza et al., 1994; Kovi et al., 2021; Peyton et al., 2020; Scattone et al., 2012; Sharp et al., 2010). The alignment with Furlong et al.'s (2014) model for covitality and the establishment of concurrent validity with the SEHM suggest the scale is another contributing factor that supports the CHKS Core's potential to evaluate SWB in youth. The literature underscored this relationship between school-related expectations and school-related outcomes such as $\mathrm{MH}$ wellness as detailed in the literature review. Other researchers have identified the relationship between high expectations and student outcomes for achievement, engagement, and perceived confidence (Akey, 2006, Ladd et al., 1999; Loukas et al., 2006; Thapa et al., 2013). The implications of this are therefore valuable to stakeholders in SBMH. 


\section{Indicators of Psychopathology by High Expectations-Adults}

An expected relationship between psychopathology and high expectations of adults was observed. Although the significance level was low across indicators of psychopathology, a descriptive review provides a valuable look at the differences among students reporting low, medium, and high levels of expectations from adults, and their experience of psychopathology. For students experiencing feelings of sadness, hopelessness, anxiousness, stress, and anger $20.3 \%(n=4,987)$ experienced low levels of high expectations compared to $12.1 \%(n=15,059)$ and $9.8 \%(n=10,330)$, who reported medium and high levels of expectations, respectively.

Results extend a similar pattern for student reports of feeling sad or hopeless almost every day. This study found $44.6 \%(n=10,928)$ of students experiencing these symptoms reported low levels of high expectations from adults. As students reported a higher experience of expectations, the percentage of students decreased by characteristics of psychopathology, with $30.6 \%(n=38,256)$ reporting medium levels of high and $25.9 \%(n=27,215)$ reporting high levels of expectations reporting these characteristics of psychopathology.

For participants reporting suicidality, reports of high expectations and contemplation of suicidality was observably meaningful. Students who reported low experiences of high expectations also reported higher rates of suicidal ideation $(27.2 \%, n=6,661)$. A gradual decrease was observed for students who rated themselves as experiencing high expectations at the medium or high level, with $15.2 \%(n=18,942)$ experiencing medium high expectations and $12.3 \%(n=12,909)$ experiencing high degrees of expectations reporting suicidality.

These results continue to align with the literature, in which a relationship is observed between SWB and psychopathology (Greenspoon \& Saklofske, 2001; Suldo \& Shaffer, 2008). 
Moreover, the results underscore the importance of school-level supports, such as high expectations, to student outcomes (Ladd et al., 1999; Thapa et al., 2013).

\section{Meaningful Participation at School}

The meaningful participation at school scale continues this study's evaluation of the CHKS Core's potential to evaluate factors related to SWB. With prior literature highlighting the role feelings of participation or contribution play in learning, achievement, and social emotional outcomes (Akey, 2006; Ladd et al., 1999; Loukas et al., 2006), the findings for the meaningful participation at school scale further highlight the utility of the CHKS Core to SBMH stakeholders. Importantly, results demonstrate the CHKS Core's potential in assessing youth SWB by establishing concurrent validity with the SEHM for Covitality index in alignment with Furlong et al. (2014) identification of school supports contributing to overall well-being, which included meaningful participation. Results of this study's analysis expressly established the meaningful participation at school scale on the CHKS Core to hold a strong, significant relationship with covitality as measured by the SEHM, with $23 \%$ of variance in the SEHM Covitality Index explainable by responses on the questions in the CHKS Core's scale.

\section{Indicators of Psychopathology by Meaningful Participation at School}

Consistent with the literature suggesting a relationship with SWB and psychopathology (Greenspoon \& Saklofske, 2001; Suldo \& Shaffer, 2008), meaningful participation showed a significant negative relationship with the CHKS Core scales correlated with the SEHM Covitality Index as a measure of SWB. Although the effect observed is small, the data continued a pattern of results important to stakeholders in SBMH. Results detail a relationship by which the level (i.e., low, medium, or high) of experienced meaningful participation produced a change in the percentage of students reporting psychopathology. Descriptively, $15.4 \%(n=14,844)$ of 
students low in their ratings of meaningful participation also reported feeling sad, hopeless, anxious stressed, or angry. This is compared to $10.2 \%(n=12,773)$ reporting medium levels of meaningful participation and 9.3\% $(n=3,094)$ reporting high levels meaningful participation and these symptoms of psychopathology.

For students reporting they felt sad or hopeless almost every day for the last 12 months, $36.8 \%(n=35,609)$ reported low levels of meaningful participation, $26.5 \%(n=33,113)$ reported medium levels of meaning participation, and 23.6\% $(n=7,860)$ reported high levels of meaningful participation. Similarly, students reporting suicidality showed reported lower levels of reported ideation by the level or reported meaningful participation (i.e., low $19.1 \%, n=$ 18,459; medium 12.8\%, $n=15,989$; high $11.5 \%, n=3,828)$.

This observed trend in declining ratings of psychopathology as levels of school-related supports for meaningful participation underscores further the value of this variable to stakeholders. The alignment with the literature suggested use of student data in this area as related to student SWB and psychopathology can be used for in consideration of a bidimensional model of youth $\mathrm{MH}$.

\section{School Connectedness}

Students' perceived feelings of personal connection to their school are associated with lower levels of MH (Shochet et al., 2006). In this study, the CHKS Core school connectedness scale was analyzed for its relationship with an established measure of SWB to determine its utility in this measurement of youth MH by evaluating SWB. The scale, which is composed of five distinct questions, evidenced a strong, significant, relationship with covitality as measured by the SEHM. With $25 \%$ of variance explainable, the results establish the scale's concurrent validity with the SEHM Covitality Index. With caring relationships, high expectations, and 
opportunities for meaningful participation all having a relationship with improving school connectedness (Austin \& O'Malley, 2012), it is not a surprising finding given those isolated scales established significance. The value of this relationship is consistent with the literature on youth $\mathrm{MH}$, which shows school connectedness to be predictive of depressive symptoms, anxiety, and overall functioning in youth (Shochet et al., 2006), as well as with decreased school conflict (Loukas et al., 2006). The utility then in this scale's measurement of SWB as part of the CHKS Core is relevant to stakeholders.

\section{Indicators of Psychopathology by School Connectedness}

The relationship between school connectedness as a correlate to SWB and psychopathology was established. Consistent with the preceding findings discussed, value in the relationship is found beyond the significance level, but instead in descriptive review. Specifically, as expected, students reporting experiences of school connectedness on a scale of low, medium, and high showed an inverse relationship with indicators of psychopathology. Descriptively, students who reported low levels of school connectedness reported higher rates of feeling sad, anxious, stressed, or angry $(23.7 \%, n=5,925)$. This relationship was observed through comparison of students' experiences at the medium and high levels of perceived school connectedness, by which $14.6 \%(n=15,888)$ of those reporting medium levels of school connectedness, and 7.4\% $(n=8,886)$ reporting high levels of perceived school connectedness, reported these characteristics of psychopathology.

Student responses for feeling sad or hopeless almost every day for the past 12 months and those reporting suicidal ideation followed a similar relationship pattern to ratings of school connectedness. Specifically, $49.9 \%(n=12,455)$ of students with low levels of school connectedness reported feeling sad or hopeless, compared to $36.7 \%(n=39,336)$ and $20.6 \%(n=$ 
24,821 ) of students reporting medium and high levels of school connectedness, respectively. Students reporting lower levels of connectedness also showed higher rates of suicidal ideation (low $31.4 \%, n=7,844$; medium 18.1\%, $n=19,387$; high 9.2\%, $n=11,067$ ).

This trend was expected and consistent with the literature on the relationship between SWB and psychopathology (Greenspoon \& Saklofske, 2001; Suldo \& Shaffer, 2008). The alignment with the literature supports use of the CHKS Core's data by stakeholders interested in applying a bidimensional model of $\mathrm{MH}$ to their interpretation of student data.

\section{Parent Involvement}

The parent involvement scale on the CHKS Core is comprised of three questions. The scale was analyzed in this study to assess its utility as a component in the measurement of SWB on the CHKS Core. Results showed a significant, positive relationship. The effect, however, was moderate compared to the other scales, which showed large effects. The amount of variance explainable in the SEHM Covitality Index by parental involvement scores was 13\%. Although lower than the other scales, these findings of a lower relationship of parent involvement to SWB is consistent with other studies' findings. In a study of the role family relationships play in SWB for adolescent youth, Lampropoulou (2018) found the relationship played a less significant role and was largely based upon the adolescent's perception of the family relationship. Although significance of the results demonstrates less strength, SBMH stakeholders may view the scales as

one contributing variable in evaluating SWB. The literature has supported parent involvement as a contributor to student positive outcomes (Hanson \& Kim, 2007; McNeely \& Falci, 2004; Waters, 2008). Importantly, Furlong et al. (2014) established family support as contributing to the second-order construct, belief-in-others, which correlated with covitality and thereafter wellbeing. 


\section{Indicators of Psychopathology by Parental Involvement}

A significant negative relationship was found among parent involvement and psychopathology. In trend with the other variables assessed for this pattern, the results have limited significance when interpreted by Cohen's (1988) standard. The results, nonetheless, hold value as did the other variables correlated with covitality on the SEHM Covitality Index when reviewed with descriptive statistics. Along all variables of psychopathology, an inverted relationship held true for students between their reported level of parent involvement and psychopathology.

For students reporting low levels of parent involvement, $18.5 \%(n=10,616)$ reported they felt sad, hopeless, anxious, stressed, or angry the previous 30 days. These reported characteristics of psychopathology declined as students reported higher ratings of parent involvement, with $11.6 \%(n=5,901)$ reporting medium levels of parent involvement and $7.8 \%$ ( $n=5,901)$ reporting high levels of parent involvementalso endorsing that they felt sad, hopeless, anxious, stressed, or angry the previous 30 days

Similar results were found for students reporting they felt sad or hopeless the last 12 months. Approximately 40.2\% $(n=23,080)$ reported these symptoms of psychopathology when also reporting low levels of parent involvement. Reported symptoms declined as students reported higher parent involvement (medium 30.7\%, $n=36,643$; high 22.3\%, $n=16$, 905).

For students experiencing suicidal ideation, the degree of parent involvement was also found to decrease student reports of ideation as ratings of parent involvement increased. Specifically, 22.4\% ( $n=12,858)$ who rated parent involvement low reported suicidality, compared to $14.8 \%(n=17,725)$ and $10.2 \%(n=7,223)$ who rated medium and high levels of involvement, respectively. 


\section{Academic Motivation}

SWB is a significant predictor of emotional, cognitive, and behavioral engagement beyond traditional psychopathology (Lyons et al., 2013) and a strong correlate to academic achievement (Datu \& King, 2018; Yao et al., 2018). In this study, the academic motivation scale, composed of four questions, showed to have a significant positive relationship with covitality on the SEHM. The effect was moderate, and approximately $22 \%$ of the variance in the SEHM Covitality Index can be explained by academic motivation scores. These results are a positive establishment of concurrent validity between the CHKS Core and the SEHM for assessment of youth SWB. Taken together with the other scales demonstrating similar results, this scale holds value in its utility to SBMH stakeholders seeking to assess youth $\mathrm{MH}$ consistent with the dual continua model.

\section{Indicators of Psychopathology by Academic Motivation}

As with the preceding five scales, a significant negative relationship was established between indicators of psychopathology and academic motivation. The magnitude of this relationship showed to be small, as it did with the preceding analyses. Nonetheless, it remains that a descriptive review of the data revealed meaning beyond correlational significance. In review, students who rated themselves as experiencing low, medium, or high levels of academic motivation showed an observable difference in their ratings of psychopathology. Specifically, as academic motivation ratings went up, reports of psychopathology declined. In descriptive review, for students who reported low levels of academic motivation, 17.8\% $(n=9,848)$ reported they felt sad, hopeless, anxious, stressed, or angry in the past 30 days, compared to $11.8 \%$ ( $n=$ $14,031)$ and $8.3 \%(n=6,526)$ who reported medium and high levels of academic motivation. 
A similar relationship was noted for students reporting feeling sad or hopeless nearly every day for the previous 12 months or experiencing suicidal ideation. For those students, $38.2 \%(n=21,107)$ who reported low levels of academic motivation also reported they felt sad or hopeless in the last 12 months. This contrasts with $30.1 \%(n=35,767)$ of those reporting medium levels of academic motivation and $25.1 \%(n=19,755)$ of those rating high for academic motivation, who also endorsed feeling sad or hopeless nearly every day for the previous 12 months.

For suicidal ideation, $22.2 \%(n=12,278)$ of students rating themselves low for academic motivation reported they seriously considered attempting suicide. These reports of this serious indicator of psychopathology declined as students reported higher levels of academic motivation. Accordingly, $14.3 \%(n=17,006)$ and $11.5 \%(n=9,021)$ of students reporting medium and high levels or academic motivation also reported suicidal ideation, respectively.

Again, the trend of declining characteristic of psychopathology based on levels of academic motivation evidenced the expected relationship between variables observed given the literature on a bidimensional model of MH (Greenspoon \& Saklofske, 2001; Suldo \& Shaffer, 2008). With SWB being further established as an indicator of cognitive and emotional engagement beyond psychopathology (Lyons et al., 2013) and a correlate to academic engagement (Datu \& King, 2018; Yao et al., 2018), these findings are notable for LEAs and other stakeholders supporting SBMH systems for youth.

\section{Summary of Results}

Overall, the analysis presented significant positive relationships for all evaluated scales hypothesized to correlate with SWB through the SEHM Covitality Index. The caring adults in school, high expectations, meaningful participation, school connectedness, parent involvement, 
and academic motivation scales showed a significant positive relationship with the SEHM Covitality Index as an established measure of student SWB at the accepted value, $\alpha=.05$. Although variance was observed in the strength of the relationship, all scales showed a moderate-to-large association with covitality (Cohen, 1988). When examining the results beyond statistical significance, the CHKS Core scales explained $22 \%-28 \%$ of the variance observed in the SEHM. The only exclusion to this is the parental involvement scale, which accounted for $13 \%$ of the observed variance.

Significant results were also found in the evaluation of relationships between the six CHKS Core scales (i.e., caring adults in school, high expectations, meaningful participation, school connectedness, parent involvement, and academic motivation) viewed as correlates to SWB on the CHKS high school core. Also, the three survey items evaluating aspects of psychopathology (i.e., students' experiences of sadness, hopelessness, anxiousness, stress, anger, and suicidality) each demonstrated a significant $(p<.001)$ inverse across all evaluated scales. A negative relationship indicated students reporting indicators of psychopathology had lower scores on the CHKS Core's subscales correlated with SWB. Although the strength of the association did not meet Cohen's (1988) standard, and the amount of variance accounted for by the variables is low $(0 \%-5 \%)$, it does suggest students with fewer school-related supports correlated with SWB experience more indicators of psychopathology.

This relationship showed the expected pattern observed for the dual continuum theory of MH in which students higher in SWB experience less distress then those low in SWB (Antaramian et al., 2010; Diener, 2013; Greenspoon \& Saklofske, 2001; Kelly, 2012; Lyons et al., 2013; Renshaw \& Cohen, 2013). Descriptive statistics emphasized these differences in experiences. An inverse relationship was observed between reports of school-related supports 
correlated with SWB and experience of indictors of psychopathology. Explicitly, students who reported experiencing medium to high degrees of school-related supports correlated with SWB also reported experiencing fewer indicators of psychopathology.

This relationship was observed across all six scales on the CHKS Core correlated with SWB (i.e., caring adults in school, high expectations, meaningful participation, school connectedness, parent involvement, and academic motivation) and all assessed indicators of psychopathology (i.e., sadness, hopelessness, anxiousness, stress, anger, and suicidality). Given this pattern of responses, all scales showed general consistency in the percentage of reported indicators of psychopathology and the level of experienced school-related supports correlated with SWB in a range of no greater than $12.6 \%$, observed between school connectedness and meaningful participation for reported experiences of feeling sad or hopeless almost every day across the last 12 months. Although school connectedness, high expectations of adults, and caring relationships descriptively showed greater influence on reported declines in indicators of psychopathology than meaningful participation, parental involvement, and academic motivation, these differences may not be statistically significant.

\section{Conclusions Based on Findings}

In total, findings in the study provide evidence that the secondary CHKS Core survey module can measure SWB, a critical component of the assessment of youth MH consistent with the dual continuum theory. Concurrent validity for the CHKS Core as a measure of youth SWB was established. A significant positive relationship was found between the six evaluated scales (i.e., caring adults in school, high expectations, meaningful participation, school connectedness, parent involvement, and academic motivation) on the CHKS Core and the SEHM Covitality Index, with the strength of the relationship meeting the threshold of a moderate to large 
association per Cohen's standard (1988) and consistent with other research establishing

concurrent validity across measures (DeSouza et al., 1994; Kovi et al., 2021; Peyton et al., 2020;

Scattone et al., 2012; Sharp et al., 2010).

In the evaluation of a relationship between correlates of SWB on the CHKS Core and indicators of psychopathology, an inverse relationship was observed as expected. Though the magnitude of the strength of the relationship between each scale correlated with SWB and indicators of psychopathology was small by Cohen's (1988) standard, the relationship identified followed the expected pattern (Greenspoon \& Saklofske, 2001; Suldo \& Shaffer, 2008), with SWB and psychopathology exhibiting an observed negative relationship. Descriptive statistics generated instead a more meaningful view of this relationship. Specifically, it was observed that the reported level (i.e., low, medium, or high) of the six CHKS Core's that correlated with SWB showed a relationship with indicators of psychopathology for students. Descriptively, the number of students endorsing indicators of psychopathology decreased as they reported higher levels of SWB, as indicated by the school related supports measured in each of the six scales. The limitations of this study, suggestions for future research, and practical implications are discussed next.

\section{Strengths and Limitations}

\section{Missing Data, Mischievous Responders, and Self Report}

Limitations of this study are found uniquely in the same areas that lend to its strength. Explicitly, although this study's strengths are its large sample size derived from a psychometrically validated scale, the size of the CHKS sample produces limitations through the amount of missing data. Problems generated by missing data center around the generalizability of the results. Missing responses reduce the overall sample size and require the interpretation of 
the results to be made with greater caution. Additional to this limitation is the nature of the survey as a self-report measure. Although "self-reports are the gold standard to assess" (Luhmann et al., 2012, p. 612) SWB, they have limitations given the high rate of mischievous responders among adolescents, which can negatively impact the research (Robinson-Cimpian, 2014). Self-reports are also sensitive to bias and exclusions due to social desirability (Fowler, 2014).

This study was strengthened by the removal of these types of responders. In applying criteria recommended by Furlong et al. (2009) and Robinson-Cimpian (2014), mischievous responders were identified and subsequently removed following a series of validity checks. Even though the results of missing data and the removal of mischievous responders decreased the sample size from 308,635 to 258,118 participants, the resulting sample analyzed remained considerable in size and, therefore, supported the generalization of the findings. Nonetheless, this assumes the remaining responders in the study answered honestly. Although the validity of selfreport measures is improved when participants can respond anonymously (McElrath, 1994), the limitations discussed herein should still be considered.

\section{Measures}

One of the most notable limitations of this study is the CHKS Core's limited assessment of psychopathology. Prior studies evaluating SWB and a bidimensional model of $\mathrm{MH}$ have used broader psychometrically validated measures of psychopathology, such as the Behavior Assessment System for Children Self Report and Teacher Report, the Self-Report Coping Scale, and the Brief Symptom Inventory (Greenspoon \& Saklofske, 2001; Lyons et al., 2013; Moore et al., 2019; Suldo \& Shaffer, 2008; Suldo et al., 2016). This study used three specific questions of the CHKS Core. These questions limited indicators of psychopathology assessed to feeling sad, 
hopeless, anxious, stressed, angry, and suicidality. The limited strength in the observed correlation between the six CHKS Core scales correlated with SWB and indicators of psychopathology may be accounted for by this limitation. It may then be a reasonable assertion that a stronger measure of psychopathology might have generated a stronger correlation. Nonetheless, although the scope of the evaluated indicators is limited, some confidence can be found in the results given depression is among the most common $\mathrm{MH}$ problems among adolescents and suicide represents the second leady cause of death among youth (Centers for Disease Control and Prevention, 2013).

\section{Sample}

The sample generated by the CHKS provides for both limitations and strengths to the study. The generalizability of the results is limited to California, from which the CHKS data were explicitly drawn. The population sample was further attenuated by students absent from school during survey administration. Students with more severe MH needs, which may have caused their absence, may have been excluded from the sample used for analysis.

Conversely, although the population samples are limited in applying the results to students in California, the CHKS sample is a highly representative sample of student data in California. Approximately 85\% of California schools participated in the 2016-2017 CHKS. Given this, a strength of the study is that data were drawn from approximately 346 participating school districts in the state.

\section{Methods of Analysis}

The study aimed to establish concurrent validity for the CHKS Core in the assessment of youth SWB. To achieve this goal, the study used correlational methods. Correlational research is a quantitative method of inquiry that evaluates the relationship between two variables by 
determining the pattern of how the two variables covary (i.e., change together; Vernoy \& Kyle, 2002). Although there are benefits in this type of analysis in the measurement of human behavior (Crawford, 2014), a weakness in correlation research is the inability to draw causal inferences from the results. Though this is a known limitation, the results are still vulnerable to misinterpretation.

As noted by Stangor (2011), "Correlational research is designed to test research hypotheses in cases where it is not possible or desirable to experimentally manipulate the independent variable of interest" (p. 178). Given the use of extant data from data collected under a nonexperimental condition, the method is most appropriate to explore the study's research questions. Correlational analysis also lends itself well to evaluating human behavior as it occurs in people's lives (Crawford, 2014), which further meets the study's aim. Although there were limitations in drawing causality in the relationship, the study used the coefficient of determination to support predictability by determining the amount of variance in one variable that can be accounted for by the other (Vernoy \& Kyle, 2002). Descriptive statistics were also used to illustrate observed variance between the negative relationship identified between the CHKS Core scales correlated with the SEHM as a measure of SWB and psychopathology. Accordingly, the resulting correlations can be interpreted beyond the observed relationship when using the coefficient of determination and descriptive statistics, lending greater value to the research community and stakeholders in SBMH.

\section{Recommendations for Future Research}

Future research is needed to further explore the CHKS Core as a measure of youth MH. For this, others should seek to replicate findings with future data sets of the CHKS beyond the 2016-2017 sample used in this study. New data sets have refinements to the measures, such as 
added questions assessing psychopathology, which could alleviate some of the weaknesses noted previously. Expanded research in this area would seek to explore further the relationships between the CHKS Core scales correlated with SWB and psychopathology. Moreover, when coupled with an assessment of psychopathology, positive evaluations of SWB can provide a more comprehensive picture of youth functioning.

It is recommended that future studies use experimental design to enable the assessment of causality. Mixed methods research is also advocated to support qualitative analysis of the relationship among school-related supports connected to SWB and psychopathology. Establishing stronger associations between factors related to SWB and predictive of psychopathology would lend greater specificity to using the CHKS for program evaluation and planning for prevention and intervention services. Given estimates that approximately $70 \%$ of youth experiencing MH problems do not access effective treatments (Adelman \& Taylor, 2012; Strein et al., 2003), greater specificity in LEAs understanding of youth MH and the ability of school-related supports to alleviate them is an important area of further research. Austin and O'Malley (2012) suggested the CHKS can be a useful guide to school districts and community stakeholders in planning prevention and intervention services for youth and holds promise for its application in youth MH. It is my belief that the CHKS holds great value in this area, and this utility can be positively expanded by continued rigorous research in the exploration and validation of the measure as such. Lastly, it is recommended the CHKS be used with a population outside of California. Limits of generalizability would diminish, and the applicability of the results would strengthen to youth $\mathrm{MH}$. 


\section{Practical Implications}

For stakeholders in SBMH, the relationship among the variables is important for systemlevel evaluation and program planning. At a more comprehensive and sophisticated level of program evaluation, LEAs desiring to evaluate and compare student data consistent with the bidimensional model of MH could use the CHKS Core as an evaluative tool of SWB alongside more robust measures of psychopathology. Research data demonstrating a range (see Table 2) of class membership distribution in the dual-factor model may be used as a reference to identify areas to target for intervention.

Descriptively, if an LEA identified a high percentage of students as troubled (i.e., low SWB and high psychopathology), they would potentially want to target interventions to bolster supports both in the six areas found correlated with SWB (i.e., caring adults in school, high expectations, meaningful participation, school connectedness, parent involvement, and academic motivation) and in employing interventions targeted at reducing symptoms of psychopathology.

More selectively, if an LEA accumulated data illustrating a high proportion of students in the vulnerable category (i.e., low psychopathology and low SWB), they may allocate resources on programs sought to bolster student SWB. Analysis of specific scales correlated with SWB may lend greater specificity in identifying areas most in need of program-level intervention. For this, LEAs may identify specific school-related supports, which bolster SWB and function as a mediator to psychopathology by using similar methods employed in this study. Specifically, LEAs can evaluate students' reported indicators of psychopathology against the reported levels (i.e., low, medium, and high) of experienced support for caring adults in school, high expectations, meaningful participation, school connectedness, parent involvement, and academic motivation. 
Areas found disproportionately low in support and showing a discernable and robust inverse relationship with reported psychopathology can help LEAs prioritize resources. They may also help determine if interventions already employed in any of these six areas are showing effectiveness as a school-wide intervention. Positively, if an LEA found data suggesting they have a high percentage of students in both the flourishing (i.e., low psychopathology and high SWB) and symptomatic but content categories (i.e., high psychopathology and high SWB), this could offer evidence that schoolwide SBMH programs are serving their student population as intended.

\section{Conclusions}

LEAs have been asked to play a critical role in youth MH (Every Student Succeeds Act, 2015; IDEA, 2004; President's Freedom Commission on Mental Health, 2003). The CHKS presents itself as a valuable resource to LEAs in both student- and program-level evaluations for youth MH (Austin \& O’Malley, 2012). Although there has been instrumental research in the field on the utility of the CHKS in the evaluation of $\mathrm{MH}$ consistent with the bidimensional model, research in this area has been isolated to the SEHM survey in the validation of covitality as a proxy to SWB (Furlong et al., 2014). As the most widely used survey among the CHKS suite (WestEd, n.d.-b), this exemplified a critical gap in the literature and a shortfall in the field of school-based mental health.

This study appropriately adds to the limited body of literature and provides guidance to LEAs and other stakeholders in the applicability of the secondary CHKS Core in the evaluation of youth SWB. The findings suggested school-related supports evaluated by the CHKS (i.e., caring adults in school, high expectations, meaningful participation, school connectedness, parent involvement, and academic motivation) provide useful information regarding students' 
state of SWB, and, although limited in magnitude, a relationship exists between these school supports and indicators of psychopathology. Overall, the study provides the CHKS high school core can measure SWB, a vital factor in the evaluation of youth $\mathrm{MH}$ consistent with the dual continuum theory. Also, findings in this study further support the dual continuum theory by displaying an inverse relationship between SWB and psychopathology (Greenspoon \& Saklofske, 2001; Suldo \& Shaffer, 2008). Importantly, the findings support the importance of SWB in SBMH interventions, with SWB showing consistency with the literature as predictive of emotional, cognitive, and behavioral engagement (Lyons et al., 2013).

This information holds utility for LEAs in the evaluation of student need and systemlevel evaluation. When coupled with an assessment of psychopathology, positive evaluations of SWB can provide a more comprehensive picture of youth functioning. This strengthened view of youth $\mathrm{MH}$ can lend instrumental support to program planning and resource allocation for both prevention and intervention efforts. It is my expressed hope that the results of this study provide support to stakeholders in the field of SBMH and stimulate additional research on the CHKS Core as an accessible tool to LEAs in their provision of SBMH services. 


\section{REFERENCES}

Adelman, H. S., \& Taylor L. (2012). Mental health in schools: Moving in new directions. Contemporary School Psychology, 16, 9-18. https://doi.org/10.1007/2FBF03340972

Akey, T. M. (2006). School context, student attitudes and behavior, and academic achievement: An exploratory analysis. MDRC. https://www.mdrc.org/sites/default/files/full_519.pdf American Academy of Pediatrics. (2000). Insurance coverage of mental health and substance abuse services for children and adolescents: A consensus statement. Pediatrics, 106, 860862. https://pediatrics.aappublications.org/content/106/4/860

Antaramian, S. P., Huebner, E. S., Hills, K. J., \& Valois, R. F. (2010). A dual-factor model of mental health: Toward a more comprehensive understanding of youth functioning. American Journal of Orthopsychiatry, 80(4), 462-472. https://doi.org/10.1111/j.19390025.2010.01049.x

Appleton, J. J., Christenson, S. L., \& Furlong, M. J. (2008). Student engagement with school: Critical conceptual and methodological issues of the construct. Psychology in the Schools, 45(5), 369-386. https://doi.org/10.1002/pits.20303

Austin, G., \& Duerr, M. (2004). Guidebook for the California Healthy Kids Survey Part I: Administration. WestEd. https://files.eric.ed.gov/fulltext/ED486326.pdf Austin, G., \& O’Malley, M. (2012). Making data-driven decisions in student support and school mental health programs: A guidebook for practice. WestEd Health \& Human Development Program. https://www.yumpu.com/en/document/view/51833045/makingdata-driven-decisions-in-student-support-and-school- 
Austin, G., Polik, J., Hanson, T., \& Zheng, C. (2018). School climate, substance use, and student well-being in California, 2015-17. Results of the Sixteenth Biennial Statewide Student Survey, Grades 7, 9, and 11. WestEd. https://data.calschls.org/resources/Biennial_State_1517.pdf

Benard, B. (2004) Resiliency: What we have learned. WestEd. https://www.wested.org/resources/resiliency-what-we-have-learned/

Burns, B. J., Costello, E. J., Angold, A., Tweed, D. Stangl, D., Farmer, E. M., \& Erkanli, A. (1995). Children's mental health service use across service sectors. Health Affairs, 14, 147-159. https://doi.org/10.1377/hlthaff.14.3.147

California Little Hoover Commission. (2001, October). Young hearts and minds: Making a commitment to children's mental health. http://lhc.ca.gov/studies/161/report161.html

Centers for Disease Control and Prevention. (2013). Mental health surveillance among children United States, 2005-2011. Morbidity and Mortality Weekly Report, 62(2), 1-35. https://www.cdc.gov/mmwr/preview/mmwrhtml/su6202a1.htm

Chao, C. C. (2017). Correlation, point-biserial. In M. Allen (Ed.), The SAGE encyclopedia of communication research methods (pp. 272-273). SAGE Publications. https://doi.org/10.4135/9781483381411

Cohen, J. (1988). Statistical power analysis for the behavioral sciences (2nd ed.). West Publishing Company.

Crawford, M. A. (2014, October 12). Strengths and limitations of correlational design. Walden University. 
Cullinan, D., Harniss, M., Epstein, M. H., \& Ryser, G. (2002). The scale for assessing emotional disturbance: Concurrent validity. Journal of Child and Family Studies, 10(4), 449-466. https://doi.org/10.1023/A:1016709407756

Datu, J. A., \& King, R. B. (2018). Subjective well-being is reciprocally associated with academic engagement: A two-wave longitudinal study. Journal of School Psychology, 69, 100-110. https://doi.org/10.1016/j.jsp.2018.05.007

Dellinger, J. B. (2017). Correlation, Spearman. In M. Allen (Ed.), The SAGE encyclopedia of communication research methods (pp. 274-275). SAGE Publications. https://doi.org/10.4135/9781483381411

Desouza, E. R., Lubin, B., \& Zanelli, J. (1994). Norms, reliability, and concurrent validity measures of the Portuguese version of the depression adjective check lists. Journal of Clinical Psychology, 50(2), 208-215. https://doi.org/10.1002/10974679(199403)50:2<208::aid-jclp2270500209>3.0.co;2-b

Diener, E. (1984). Subjective well-being. Psychological Bulletin, 95(3), 542-575. https://doi.org/10.1037/0033-2909.95.3.542

Diener, E. (2013). The remarkable changes in the science of subjective well-being. Perspectives on Psychological Science, 8(6), 663-666. https://doi.org/10.1177/1745691613507583

Every Student Succeeds Act of 2015, Pub. L. No. 114-95 § 114 Stat. 1177 (2015-2016).

Fredkove, W. M., Gower, A. L., \& Renee, E. S. (2019). Association among internal assets, bullying and emotional distress in eight grade students. Journal of School Health, 89(11), 1-17. https://doi.org/10.1111/josh.12833 
Furlong, M. J., Ritchey, K. M., \& O’Brennan, L. M. (2009). Developing norms for the California Resilience Youth Development module: Internal assets and school resources subscales. The California School Psychologist, 14(1), 35-46. https://doi.org/10.1007/bf03340949

Furlong, M. J., You, S., Renshaw, T. L., Smith, D. C., \& O’Malley, M. D. (2014). Preliminary development and validation of the social and emotional health survey for secondary school students. Social Indicators Research, 117(3), 1011-1032. https://doi.org/10.1007/s11205-013-0373-0

Gignac, G. E., \& Szodorai, E. T. (2016). Effect size guidelines for individual differences researchers. Personality and Individual Differences, 102, 74-78. https://doi.org/10.1016/j.paid.2016.06.069

Greenspoon, P. J. \& Salklofske, D. H. (2001). Toward an integration of subjective well-being and psychopathology. Social Indicators of Research, 54(1), 81-108. https://www.jstor.org/stable/27526929

Hanson, T. L., \& Kim, J. O. (2007). Measuring resilience and youth development: The psychometric properties of the Healthy Kids Survey (Issues \& Answers Report, REL 2007-No. 034). U.S. Department of Education, Institute of Education Sciences, National Center for Education Evaluation and Regional Assistance, Regional Educational Laboratory West. http://ies.ed.gov/ncee/edlabs

Howell, D. C. (2013). Fundamental statistics for the behavioral sciences (8th ed.). Brooks/ColeThompson Learning.

Hughes, M. R., Gaines, J. S., \& Pryor, D. W. (2015). Staying away from school. Youth Violence and Juvenile Justice, 13(3), 270-290. https://doi.org/10.1177/1541204014538067

Individuals With Disabilities Education Act [IDEA], 20 U.S.C. § 1400 (2004) 
Institute of Medicine. (2009). Preventing mental, emotional, and behavioral disorders among young people: Progress and possibilities. National Academies Press. https://doi.org/10.17226/12480

Jahoda, M. (1958). Current concepts of positive mental health. Basic Books.

Jones, C. N., You, S., \& Furlong, M. J. (2013). A Preliminary examination of covitality as integrated well-being in college students. Social Indicators Research, 111(2), 511-526. https://doi.org/10.1007/s11205-012-0017-9

Kandel, D. B., Johnson, J. G., Bird, H. R., Canino, G. C., Goodman, S. H., Lahey, B. B., Regier, D. A., \& Schwab-Stone, M. (1997). Psychiatric disorders associated with substance use among children and adolescents: Findings from the methods for the epidemiology of child and adolescent mental disorders (MECA) study. Journal of Abnormal Child Psychology, 25(2), 121-132. https://doi.org/10.1023/a:1025779412167

Kaplan, D. W., Calonge, B. N., Guernsey, B. P., \& Hanrahan, M. B. (1998). Managed care and school-based health centers: Use of health services. Archives of Pediatric Adolescent Medicine, 152(1), 25-33. https://doi.org/10.1001/archpedi.152.1.25

Kelly, R. M., Hills, K. J., Huebner, E. S., \& McQuillin, S. D. (2012). The longitudinal stability and dynamics of group membership in the dual-factor model of mental health. Canadian Journal of School Psychology, 27(4), 337-355. https://doi.org/10.1177/0829573512458505

Kemery, E. R., Griffeth, R. W., \& Dunlap, W. P. (1988). Correction for variance restriction in point-biserial correlations. Journal of Applied Psychology, 73(4), 688-691. https://doi.org/10.1177/0829573512458505 
Kinderman, P. (2005). A psychological model of mental disorder. Harvard Review of Psychiatry, 13(4), 206-217. https://doi.org/10.1080/10673220500243349

Kline, R. B. (2010). Principles and practice of structural equation modeling (3rd ed.). The Guilford Press.

Kövi, Z., Hittner, J. B., Mirnics, Z., Grezsa, F., Smohai, M., Jakšić, N., Meszaros, V., Rozsa, S., Vargha, A., Tanyi, Z., \& Vass, Z. (2021).

Concurrent validity of the sixty-second drawing test in measuring high-schoolers' close relationships and depression. Rorschachiana, 42(1), 52-71. https://doi.org/10.1027/11925604/a000141

Lacoe, J. (2016). Too scared to learn? The academic consequences of feeling unsafe in the classroom. Urban Education, 55(1), 1385-1418 https://doi.org/10.1177/0042085916674059

Ladd, G. W., Birch, S. H., \& Buhs, E. S. (1999). Children's' social and scholastic lives in kindergarten: Related spheres of influence? Child Development, 70(6), 1373-1400. https://doi.org/10.1111/1467-8624.00101

Lampropoulou, A. (2018). Personality, school, and family: What is their role in adolescents' subjective well-being? Journal of Adolescence. 67, 12-21. https://doi.org/10.1016/j.adolescence.2018.05.013

La Salle, T., George, P. H., McCoach, D. B., Polk, T., \& Evanovich, L. L. (2018). An examination of school climate, victimization, and mental health problems among middle school students self-identifying with emotional and behavioral disorders. Behavioral Disorders, 43(3), 383-392. https://doi.org/10.1177/0198742918768045 
Lenzi, M., Sharkey, J., Furlong, M. J., Mayworm, A., Hunnicutt, K., \& Vieno, A. (2017). School sense of community, teacher support, and students' school safety perceptions. American Journal of Community Psychology, 60(3-4), 527-537. https://doi.org/10.1002/ajcp.12174

Long, R. F., Huebner, E. S., Wedell, D. H., \& Hills, K. J. (2012). Measuring school-related subjective well-being in adolescents. American Journal of Orthopsychiatry, 82(1), 50-60. https://doi.org/10.1111/j.1939-0025.2011.01130.x

Loukas, A., Suzuki, R., \& Horton, K. D. (2006). Examining school connectedness as a mediator of school climate effects. Journal of Research on Adolescence, 16(3), 491-502. https://doi.org/10.1111/j.1532-7795.2006.00504.x

Luhmann, M., Hofmann, W., Eid, M., \& Lucas, R. E. (2012). Journal of Personality and Social Psychology, 102(3), 592-615. https://doi.org/10.1037/a0025948

Lyons, M. D., Huebner, E. S., \& Hills, K. J. (2013). The dual-factor model of mental health: A short-term longitudinal study of school-related outcomes. Social Indicators Research, 114(2), 549-565. https://doi.org/10.1007/s11205-012-0161-2

Masten, A. (2014). Ordinary magic: Resilience in development. The Guilford Press.

Maul, A. (2018). Validity. The SAGE encyclopedia of educational research, measurement, and evaluation. SAGE Publications. https://doi.org/10.4135/9781506326139

McElrath, K. A. (1994). A comparison of two methods for examining self-reported drug use. International Journal of Addiction, 29(4), 517-524. https://doi.org/10.3109/10826089409047397 
McNeely, C. A., \& Falci, C. (2004). School connectedness and the transition into and out of health-risk behavior among adolescents: A comparison of social belonging and teacher support. Journal of School Health, 74, 284-293. https://doi.org/10.1111/j.17461561.2004.tb08285.x

Merikangas, K. R., He, J., Burstein, M., Swanson, S. A., Avenevoli, S., Cui, L., Benjet, C., Georgiades, K., \& Swendsen, J. (2010). Lifetime prevalence of mental disorders in US adolescents: Results from the National Comorbidity Study-Adolescent Supplement (NCS-A). Journal of American Academy Child Adolescent Psychiatry, 49(10), 980-989. https://doi.org/10.1016/j.jaac.2010.05.017

National Alliance on Mental Illness. (n.d.). Mental health facts - Children and teens. http://www.nami.org/getattachment/Learn-More/Mental-Health-by-theNumbers/childrenmhfacts.pdf

National Center for Education Statistics. (1997). NELS: 88 survey item report (No. NCES 97052). U.S. Department of Education. https://nces.ed.gov/pubs97/97052.pdf

National School Climate Council. (2007). The school climate challenge: Narrowing the gap between school climate research and school climate policy, practice guidelines, and teacher education policy. http://www.ecs.org/school-climate

Nijs, M. M., Bun, C. J. E., Tempelaar, W. M., Wit, N. J. D., Burger, H., Plevier, C. M., \& Boks, M. P. M. (2014). Perceived school safety is strongly associated with adolescent mental health problems. Community Mental Health Journal, 50(2), 127-134. https://doi.org/10.1007/s10597-013-9599-1 
Ozer, E. J., \& Weinstein, R. S. (2004). Urban adolescents' exposure to community violence: The role of support, school safety, and social constraints in a school-based sample of boys and girls. Journal of Clinical Child and Adolescent Psychology, 33(3), 463-476. https://doi.org/10.1207/s15374424jccp3303_4

Park, N. (2004). The role of subjective well-being in positive youth development. The Annals of the American Academy of Political and Social Science, 591(1), 25-39. https://doi.org/10.1177/0002716203260078

Peyton, C., Msall, M. E., Wroblewski, K., Rogers, E. E., Kohn, M., \& Glass, H. C. (2020). Concurrent validity of the Warner Initial Developmental Evaluation of Adaptive and Functional Skills and the Bayley Scales of Infant and Toddler Development, Third Edition. Developmental Medicine \& Child Neurology, 63(3), 349-354. https://doi.org/10.1111/dmcn.14737

Reardon, T., Harvey, K., Baranowska, M., O’Brien, D., Smith, L., \& Creswell, C. (2017). What do parents perceive are the barriers and facilitators to accessing psychological treatment for mental health problems in children and adolescents? A systematic review of qualitative and quantitative studies. European Child \& Adolescent Psychiatry, 26(6), 623-647. https://doi.org/10.1007/s00787-016-0930-6

Renshaw, T. L., \& Cohen, A. S. (2014). Life satisfaction as a distinguishing factor of college student functioning: Further validation of the two-continua model of mental health. Social Indicators Research, 117, 319-334. https://doi.org/10.1007/s11205-013-0342-7

Robinson-Cimpian, J. P. (2014). Inaccurate estimation of disparities due to mischievous responders: Several suggestions to assess conclusions. American Education Research Association, 43(4), 171-185. https://doi.org/10.3102/0013189X14534297 
Rodríguez-Fernández, A., Ramos-Díaz, E., \& Axpe-Saez, I. (2018). The role of resilience and psychological well-being in school engagement and perceived academic performance: An exploratory model to improve academic achievement. Health and Academic Achievement. https://doi.org/10.5772/intechopen.73580

Ryff, C. D., \& Singer, B. (1998). The contours of positive human health. Psychological Inquiry, 9(1), 1-28. https://doi.org/10.1207/s15327965pli0901_1

Salkind, N. J. (Ed.). (2017). Encyclopedia of research design - Spearman rank order correlation. SAGE Publications. https://doi.org/10.4135/9781412961288

Salle, T. L., George, H. P., McCoach, D. B., Polk, T., \& Evanovich, L. L. (2018). An examination of school climate, victimization, and mental health problems among middle school students self-identifying with emotional and behavioral disorders. Behavioral Disorders, 43(3), 383-392. https://doi.org/10.1177/0198742918768045

Satcher, D. (2004). School-based mental health services. Pediatrics, 113(6), 1839-1845. https://doi.org/10.1542/peds.113.6.1839

Scattone, D., Raggio, D. J., \& May, W. (2012). Brief report: Concurrent validity of the Leiter-R and KBIT-2 Scales of Nonverbal Intelligence for Children with Autism and Language Impairments. Journal of Autism and Developmental Disorders, 42(11), 2486-2490. https://doi.org/10.1007/s10803-012-1495-y

Sharp, C., Mosko, O., Chang, B., \& Ha, C. (2010). The cross-informant concordance and concurrent validity of the Borderline Personality Features Scale for Children in a community sample of boys. Clinical Child Psychology and Psychiatry, 16(3), 335-349. https://doi.org/10.1177/1359104510366279 
Shochet, I. M., Dadds, M. R., Ham, D., \& Montague, R. (2006). School connectedness is an underemphasized parameter in adolescent mental health: Results of a community prediction study. Journal of Clinical Child \& Adolescent Psychology, 35(2), 170-179. https://doi.org/10.1207/s15374424jccp3502_1

Stangor, C. (2011). Research methods for the behavioral sciences. Cengage Learning.

Stoep, A. V., Weiss, N. S., Kuo, E. S., Cheney, D., \& Cohen, P. (2003). What proportion of failure to complete secondary school in the US population is attributable to adolescent psychiatric disorder? The Journal of Behavioral Health Services and Research, 30(1), $119-124$.

Strein, W., Hoagwood, K., \& Cohn, A. (2003). School psychology: A public health perspective: I. Prevention, populations, and systems change. Journal of School Psychology, 41(1), 2338. https://doi.org/10.1016/S0022-4405(02)00142-5

Substance Abuse and Mental Health Services Administration. (2011). Results from the 2010 National Survey on Drug Use and Health: Summary of national findings, NSDUH Series H-41 (HHS Publication No. [SMA] 11-4658). Substance Abuse and Mental Health Services Administration. https://www.samhsa.gov/data/sites/default/files/NSDUHNationalFindingsResults2010web/2k10ResultsRev/NSDUHresultsRev2010.pdf

Suldo, S. M., \& Shaffer, E. J. (2008). Looking beyond psychopathology: The dual-factor model of mental health in youth. School Psychology Review, 37(1), 52-68. https://doi.org/10.1080/02796015.2008.12087908 
Suldo, S. M., Thalji-Raitano, A., Kiefer, S. M., \& Ferron, J. M. (2016). Conceptualizing high school students' mental health through a dual-factor model. School Psychology Review, 45(4), 434-457. https://doi.org/10.17105/SPR45-4.434-457

Thapa, A., Cohen, J., Guffey, S., \& Higgins-D’Alessandro, A. (2013). A review of school climate research. Review of Educational Research, 83(3), 357-385. https://doi.org/10.3102/0034654313483907

U.S. President's New Freedom Commission on Mental Health. (2003). Achieving the promise: Transforming mental health care in America: Final report. https://govinfo.library.unt.edu/mentalhealthcommission/reports/FinalReport/FullReport1.htm

Vernoy, M. W., \& Kyle, D. (2002). Behavioral statistics in action (3rd ed.). McGraw Hill Education.

Waters, S., \& Cross, D. (2010). Measuring students' connectedness to school, teachers, and family: Validation of three scales. School Psychology Quarterly, 25(3), 164-177. https://doi.org/10.1037/a0020942

Weiss, A., King, J. E., \& Enns, R. M. (2002). Subjective well-being is heritable and genetically correlated with dominance in chimpanzees (Pan troglodytes). Journal of Personality and Social Psychology, 83(5), 1141-1149. https://doi.org/10.1037/0022-3514.83.5.1141

Weist, M. D., Goldstein, A., Morris, L., \& Bryant, T. (2003). Integrating expanded school mental health programs and school-based health centers. Psychology in the Schools, 40(3), 297308. https://doi.org/10.1002/pits.10089 
West, C. P., \& Beckman, T. J. (2018). Concurrent validity. In Sage Publications, The SAGE encyclopedia of educational research, measurement, and evaluation (pp. 1-3). SAGE Publications. https://doi.org/10.4135/9781506326139

WestEd. (n.d.-a.). California Healthy Kids Survey (CHKS). https://www.wested.org/project/california-healthy-kids-survey-chks/

WestEd. (n.d.-b.). California Healthy Kids Survey Website. https://calschls.org/about/thesurveys/\#chks

Wiley, D. C., \& Cory, A. C. (2013). Encyclopedia of school health. SAGE Publications.

World Health Organization. (2013). Comprehensive mental health action plan 2013-2020. https://www.who.int/mental_health/action_plan_2013/en/

You, S., Furlong, M., Felix, E., \& O’Malley, M. (2015). Validation of the Social and Emotional Health Survey for five sociocultural groups: Multigroup invariance and latent mean analyses. Psychology in the Schools, 52(4), 349-362. https://doi.org/10.1002/pits.21828

You, S., Dowdy, E., Furlong, M. J., \& Renshaw, T. L. (2014). Further validation of the Social and Emotional Health Survey for high school students. Applied Research in Quality of Life, 9, 997-1015. https://doi.org/10.1007/s11482-013-9282-2 


\title{
APPENDICES
}

Appendix A: California Healthy Kids Survey-High School Core

\author{
CALIFORNIA healthykids SUR VEY \\ Core Module
}

High School Questionnaire

2016-2017

This survey asks about your behavior, experiences, and attitudes related to your school, health, and well-being. It includes questions about use of alcohol, tobacco, and other drugs, and about bullying and violence.

You do not have to answer these questions, but your answers will be very helpful in improving school and health programs. You will be able to answer whether or not you have done or experienced any of these things.

Please do not write your name on this form or the answer sheet. Do not identify yourself in any other way.

Please mark all of your answers on the answer sheet. Fill in the bubbles neatly with a \#2 pencil. Do not write on the questionnaire. Mark only one answer unless told to "Mark All That Apply."

This survey asks about things you may have done during different periods of time, such as during your lifetime (you ever did something), or the past 12 months, or 30 days. Each provides different information. Please pay careful attention to these time periods.

Thank you for taking this survey!

California Healthy Kids Survey (C2016 CA Dept. of Ed. 


\section{A L I F OR I A healthykids S U R VE Y Core Module}

Begin by writing your school's name at the top of the answer sheet.

1. Fill in the bubble for the letter "H."

2. Fill in the bubble for the letter "J."

\section{Next, we would like some background information about you.}

3. What is your sex?

$\begin{array}{ll}\text { A) } & \text { Male } \\ \text { B) Female }\end{array}$

4. What grade are you in?
A) 6th grade
B) 7th grade
C) 8th grade
D) 9th grade
F) 11th grade
G) 12th grade
E) 10th grade
H) Other grade
I) Ungraded

5. Are you of Hispanic or Latino origin?
A) No
B) Yes

6. What is your race?
A) American Indian or Alaska Native
D) Native Hawaiian or Pacific Islander
B) Asian
C) Black or African American
E) White
F) Mixed (two or more) races 


\section{A L I FOR N I A healthykids S U R V E \\ Core Module}

7. If you are Asian or Pacific Islander, which groups best describe you? (Mark All That Apply.) If you are not of Asian/Pacific Islander background, mark "A) Does not apply." Pacific Islander
B) Asian Indian
C) Cambodian
D) Chinese
E) Filipino
F) Hmong
G) Japanese

A) Does not apply; I am not Asian or

H) Korean

I) Laotian

J) Vietnamese

K) Native Hawaiian, Guamanian, Samoan, Tahitian, or other Pacific Islander

L) Other Asian

8. What best describes where you live? A home includes a house, apartment, trailer, or mobile home.
A) A home with one or more parents or guardian
B) Other relative's home
C) A home with more than one family
D) Friend's home
E) Foster home, group care, or waiting placement
F) Hotel or motel
G) Shelter, car, campground, or other transitional or temporary housing
H) Other living arrangement

9. What is the highest level of education your parents or guardians completed? (Mark the educational level of the parent or guardian who went the furthest in school.)
A) Did not finish high school
D) Graduated from college
B) Graduated from high school
E) Don't know
C) Attended college but did not complete four-year degree

10. Do you receive free or reduced-price lunches at school? (Receiving free or reduced-price lunches means that lunch at school is provided to you for free or you pay less for it.)
A) No
B) Yes
C) Don't know

11. In the past three years, were you part of the Migrant Education Program or did your family move to find seasonal or temporary work in agriculture or fishing?
A) No
B) Yes
C) Don't know 


\section{A L I F OR N I A healthykids SUR VE Y \\ Core Module}

12. What language is spoken most of the time in your home?
A) English
F) Tagalog
B) Spanish
G) Vietnamese
C) Mandarin
H) Korean
D) Cantonese
I) Other

How well do you understand, speak, read, and write English?

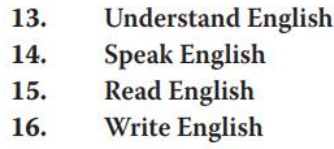

\begin{tabular}{|c|c|c|c|}
\hline $\begin{array}{l}\text { Very } \\
\text { Well }\end{array}$ & Well & $\begin{array}{l}\text { Not } \\
\text { Well }\end{array}$ & $\begin{array}{l}\text { Not } \\
\text { At All }\end{array}$ \\
\hline A & B & C & D \\
\hline A & B & $\mathrm{C}$ & D \\
\hline A & B & $\mathrm{C}$ & D \\
\hline A & B & C & D \\
\hline
\end{tabular}

17. How many days a week do you usually go to your school's after school program?
A) 0 days
E) 4 days
B) 1 day
F) 5 days
C) 2 days
D) 3 days

18. During the past 12 months, how would you describe the grades you mostly received in school?
A) Mostly A's
E) Mostly C's
B) A's and B's
F) C's and D's
C) Mostly B's
G) Mostly D's
D) B's and C's
H) Mostly F's

19. During the past 12 months, about how many times did you skip school or cut classes?
A) 0 times
D) Once a month
B) 1-2 times
E) Once a week
C) A few times
F) More than once a week 


\section{A L IFORNIA healthykids SUR VE Y \\ Core Module}

20. In the past $\mathbf{3 0}$ days, did you miss school for any of the following reasons? (Mark All That Apply.)
A) Does not apply, I didn't miss any school
H) Wanted to spend time with friends who don't go to your school
B) Illness (feeling physically sick), including problems with breathing or your teeth
I) Wanted to use alcohol or drugs
J) Were behind in schoolwork or weren't prepared for a test or class assignment
C) Felt very sad, hopeless, anxious, stressed, or angry
D) Didn't get enough sleep
E) Didn't feel safe at school
F) Had to work
K) Were bored with or uninterested in school
L) Were suspended
M) Other reason

G) Had to take care of or help a family member or friend

How strongly do you agree or disagree with the following statements?

21. I feel close to people at this school.

22. I am happy to be at this school.

23. I feel like I am part of this school.

24. The teachers at this school treat students fairly.

25. I feel safe in my school.

26. My school is usually clean and tidy.

27. Teachers at this school communicate with parents about what students are expected to learn in class.

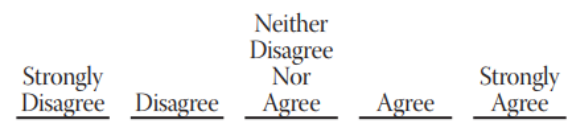

28. Parents feel welcome to participate at this school.

29. School staff takes parent concerns seriously.

30. I try hard to make sure that I am good at my schoolwork.

31. I try hard at school because I am interested in my work.

32. I work hard to try to understand new things at school.

33. I am always trying to do better in my schoolwork.

$\underline{\text { Disagree }} \underline{\text { Disagree }}$

Agree




\section{A L I F OR N I A healthykids S U R V E Y}

Core Module

Please mark on your answer sheet how TRUE you feel each of the following statements is about your SCHOOL and things you might do there.

At my school, there is a teacher or some other adult ...

34. who really cares about me.

35. who tells me when I do a good job.

36. who notices when I'm not there.

37. who always wants me to do my best.

38. who listens to me when I have something to say.

39. who believes that I will be a success.

\begin{tabular}{|c|c|c|c|}
\hline $\begin{array}{c}\text { Not At All } \\
\text { True } \\
\end{array}$ & $\begin{array}{c}\text { A Little } \\
\text { True }\end{array}$ & $\begin{array}{l}\text { Pretty Much } \\
\text { True } \\
\end{array}$ & $\begin{array}{c}\text { Very Much } \\
\text { True }\end{array}$ \\
\hline A & B & C & D \\
\hline A & B & C & D \\
\hline A & B & C & D \\
\hline A & B & C & D \\
\hline A & B & C & D \\
\hline A & B & C & D \\
\hline
\end{tabular}

At school, ...

40. I do interesting activities.

41. I help decide things like class activities or rules.

42. I do things that make a difference.

\begin{tabular}{|c|c|c|c|}
\hline $\begin{array}{l}\text { Not At All } \\
\text { True }\end{array}$ & $\begin{array}{c}\text { A Little } \\
\text { True }\end{array}$ & $\begin{array}{l}\text { Pretty Much } \\
\text { True } \\
\end{array}$ & $\begin{array}{c}\text { Very Much } \\
\text { True }\end{array}$ \\
\hline A & B & C & D \\
\hline A & B & C & D \\
\hline A & B & C & $\mathrm{D}$ \\
\hline
\end{tabular}




\section{A L I F OR N I A healthy kids $S U R V E Y$}

\section{Core Module}

The next questions ask about the use of alcohol, tobacco, marijuana, and other drugs, including pills or medications, to get "high" or for reasons other than medical (without a doctor's order).

\section{Keep the following definitions in mind:}

- One drink of ALCOHOL, or alcoholic drink (beverage), means one regular size can/bottle of beer or wine cooler, one glass of wine, one mixed drink, or one shot glass of liquor.

- Questions about alcohol do not include drinking a few sips of wine for religious purposes.

- DRUG means any substance other than alcohol or tobacco, including pills and medications, used to get "high" ("loaded", "stoned", or "wasted") or for purposes other than prescribed by a doctor.

During your life, how many times have you used the following substances?

\section{A whole cigarette \\ 44. Smokeless tobacco (dip, chew, or snuff) \\ 45. Electronic cigarettes, e-cigarettes, or other vaping device such as e-hookah, hookah pens, or vape} pens

46. One full drink of alcohol (such as a can of beer, glass of wine, wine cooler, or shot of liquor)

47. Marijuana (pot, weed, grass, hash, bud)

48. Inhalants (things you sniff, huff, or breathe to get "high" such as glue, paint, aerosol sprays, gasoline, poppers, gases)

49. Cocaine, Methamphetamine, or any amphetamines (meth, speed, crystal, crank, ice)

50. Derbisol (DB, derbs, dirt)

51. Ecstasy, LSD, or other psychedelics (acid, mescaline, peyote, mushrooms)

52. Prescription pain medication (Vicodin ${ }^{\mathrm{ne}}$, OxyContin $^{\mathrm{nt}}$, Percodan ${ }^{\mathrm{nt}}$, Lortab $\left.{ }^{\mathrm{tx}}\right)$, tranquilizers,

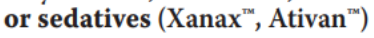

53. Diet Pills (Didrex, Dexedrine, Zinadrine, Skittles, M\&M's)

54. Ritalin $^{\mathrm{Tw}}$ or Adderall ${ }^{\mathrm{Tw}}$ (JIF, R-ball, Skippy) or other prescription stimulant

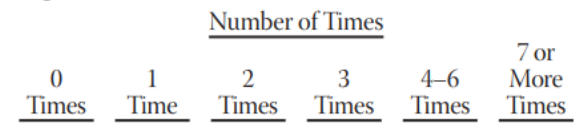

$\begin{array}{llllll}\text { A } & \text { B } & \text { C } & \text { D } & \text { E } & \text { F } \\ \text { A } & \text { B } & \text { C } & \text { D } & \text { E } & \text { F } \\ \text { A } & \text { B } & \text { C } & \text { D } & \text { E } & \text { F }\end{array}$

$\begin{array}{llllll}\text { A } & \text { B } & \text { C } & \text { D } & \text { E } & \text { F } \\ \text { A } & \text { B } & \text { C } & \text { D } & \text { E } & \text { F } \\ \text { A } & \text { B } & \text { C } & \text { D } & \text { E } & \text { F }\end{array}$

A $\quad$ B $\quad$ C $\quad$ D $\quad$ E $\quad$ F

$\begin{array}{lllllll}\text { A } & \text { B } & \text { C } & \text { D } & \text { E } & \text { F }\end{array}$

$\begin{array}{llllll}\text { A } & \text { B } & \text { C } & \text { D } & \text { E } & \text { F }\end{array}$

$\begin{array}{llllll}\text { A } & \text { B } & \text { C } & \text { D } & \text { E } & \text { F }\end{array}$

$\begin{array}{lllllll}\text { A } & \text { B } & \text { C } & \text { D } & \text { E } & \text { F }\end{array}$

$\begin{array}{lllllll}\text { A } & \text { B } & \text { C } & \text { D } & \text { E } & \text { F }\end{array}$ 
C A L I F OR N A healthykids $S U R V E Y$

Core Module

During your life, how many times have you used the following substances? Cough, Sudafed, TheraFlu, Tylenol Cough) or other

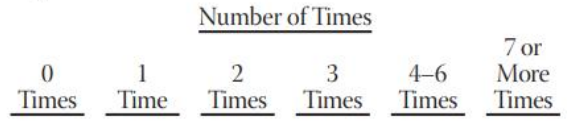
over-the-counter medicines

56. Any other drug, pill, or medicine to get "high" or for other than medical reasons
A
B
C
D
E $\quad F$
A $\quad$ B
C D
E $\quad$ F

During your life, how many times have you been ...

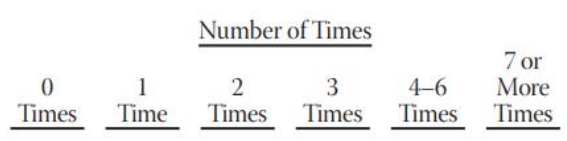

57. very drunk or sick after drinking alcohol?

58. "high" (loaded, stoned, or wasted) from using drugs?

59. drunk on alcohol or "high" on drugs on school property?

$\begin{array}{llllll}\text { A } & \text { B } & \text { C } & \text { D } & \text { E } & \text { F } \\ \text { A } & \text { B } & \text { C } & \text { D } & \text { E } & \text { F } \\ & & & & & \\ \text { A } & \text { B } & \text { C } & \text { D } & \text { E } & \text { F }\end{array}$

During the past 30 days, on how many days did you use ...

60. cigarettes?

61. smokeless tobacco (dip, chew, or snuff)?

62. electronic cigarettes, e-cigarettes, or other vaping device such as e-hookah, hookah pens, or vape pens?

\begin{tabular}{|c|c|c|c|c|c|}
\hline $\begin{array}{c}0 \\
\text { Days }\end{array}$ & $\begin{array}{c}1 \\
\text { Day }\end{array}$ & $\begin{array}{c}2 \\
\text { Days }\end{array}$ & $\begin{array}{l}3-9 \\
\text { Days }\end{array}$ & $\begin{array}{c}10-19 \\
\text { Days }\end{array}$ & $\begin{array}{c}20-30 \\
\text { Days }\end{array}$ \\
\hline A & B & C & D & $E$ & $\mathrm{~F}$ \\
\hline A & B & C & D & $E$ & $\mathrm{~F}$ \\
\hline A & B & C & D & $E$ & $\mathrm{~F}$ \\
\hline
\end{tabular}

63. at least one drink of alcohol?

64. five or more drinks of alcohol in a row, that is, $\begin{array}{llllll}\text { A } & \text { B } & \text { C } & \text { D } & \text { E } & \text { F }\end{array}$ within a couple of hours?

65. marijuana (pot, weed, grass, hash, bud)?

66. inhalants (things you sniff, huff, or breathe to get "high")?

67. prescription medications to get "high" or for reasons other than prescribed (such as Vicodin ${ }^{\mathrm{tw}}$, OxyContin ${ }^{\mathrm{nw}}$, Percodan $^{\mathrm{nw}}$, Ritalin $^{\mathrm{n \omega}}$, Adderall ${ }^{\mathrm{Tw}}$, Xanax $\left.^{\mathrm{nt}}\right)$ ?

68. any other drug, pill, or medicine to get "high" or for other than medical reasons?

69. two or more substances at the same time (for example, alcohol with marijuana, ecstasy with mushrooms)? 


\section{A L I FOR N I A healthykids $S U R V E Y$}

\section{Core Module}

During the past 30 days, on how many days on school property did you ...

70. smoke cigarettes?

71. use smokeless tobacco (dip, chew, or snuff)?

72. use electronic cigarettes, e-cigarettes, or other vaping device such as e-hookah, hookah pens, or

\begin{tabular}{|c|c|c|c|c|c|}
\hline $\begin{array}{c}0 \\
\text { Days }\end{array}$ & $\begin{array}{c}1 \\
\text { Day }\end{array}$ & $\begin{array}{c}2 \\
\text { Days }\end{array}$ & $\begin{array}{l}3-9 \\
\text { Days }\end{array}$ & $\begin{array}{c}10-19 \\
\text { Days }\end{array}$ & $\begin{array}{c}20-30 \\
\text { Days }\end{array}$ \\
\hline A & B & C & D & $E$ & $\mathrm{~F}$ \\
\hline A & B & C & D & $E$ & $F$ \\
\hline A & B & C & D & $E$ & $F$ \\
\hline
\end{tabular}

73. have at least one drink of alcohol?

74. smoke marijuana?

75. use any other drug, pill, or medicine to get "high" or for other than medical reasons?

$\begin{array}{llllll}\text { A } & \text { B } & \text { C } & \text { D } & \text { E } & \text { F } \\ \text { A } & \text { B } & \text { C } & \text { D } & \text { E } & \text { F } \\ \text { A } & \text { B } & \text { C } & \text { D } & \text { E } & \text { F }\end{array}$

How much do people risk harming themselves physically and in other ways when they do the following?

77. Smoke 1-2 packs of cigarettes each day

78. Drink alcohol occasionally

79. Have five or more drinks of an alcoholic beverage once or twice a week

80. Smoke marijuana occasionally

81. Smoke marijuana once or twice a week

\begin{tabular}{|c|c|c|c|}
\hline \multirow[b]{2}{*}{ Great } & \multicolumn{2}{|c|}{ How Much Risk or Harm } & \multirow[b]{2}{*}{ None } \\
\hline & Moderate & Slight & \\
\hline A & B & $\mathrm{C}$ & D \\
\hline A & B & $\mathrm{C}$ & D \\
\hline A & B & $\mathrm{C}$ & D \\
\hline A & B & $\mathrm{C}$ & D \\
\hline A & B & $\mathrm{C}$ & $\mathrm{D}$ \\
\hline A & B & $\mathrm{C}$ & D \\
\hline
\end{tabular}

How difficult is it for students in your grade to get any of the following substances if they really want them?

82. Cigarettes

83. Alcohol

84. Marijuana

How many times have you tried to quit or stop using...

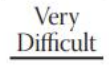

Difficult Difficult $\begin{array}{r}\text { Fairly } \\ \text { Easy } \\ \hline\end{array}$$$
\text { B }
$$$$
\text { A } \quad \text { B }
$$

A B

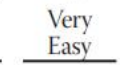

Don't

$\mathrm{C}$
$\mathrm{C}$
$\mathrm{C}$
Know

$\begin{array}{ll}D & \text { E } \\ D & \text { E }\end{array}$

85. cigarettes?
86. alcohol?
87. marijuana?
Does Not

Apply,

Use
A

\begin{tabular}{c}
0 Times \\
\hline B \\
B \\
B
\end{tabular}

\begin{tabular}{|c|c|c|}
\hline 1 Time & $\begin{array}{c}2-3 \\
\text { Times }\end{array}$ & $\begin{array}{l}4 \text { or } \\
\text { More } \\
\text { Times }\end{array}$ \\
\hline C & D & $E$ \\
\hline C & D & E \\
\hline C & D & E \\
\hline
\end{tabular}




\section{A L I FOR I A healthykids S U R VE Y Core Module}

88. During your life, how many times have you ever driven a car when you had been drinking alcohol, or been in a car driven by a friend when he or she had been drinking?
A) Never
B) 1 time
C) 2 times
D) 3 to 6 times
E) 7 or more times

\section{Next are questions about violence, safety, harassment, \& bullying on school property.}

89. How safe do you feel when you are at school?
A) Very safe
B) Safe
C) Neither safe nor unsafe
D) Unsafe
E) Very unsafe

During the past 12 months, how many times on school property have you...

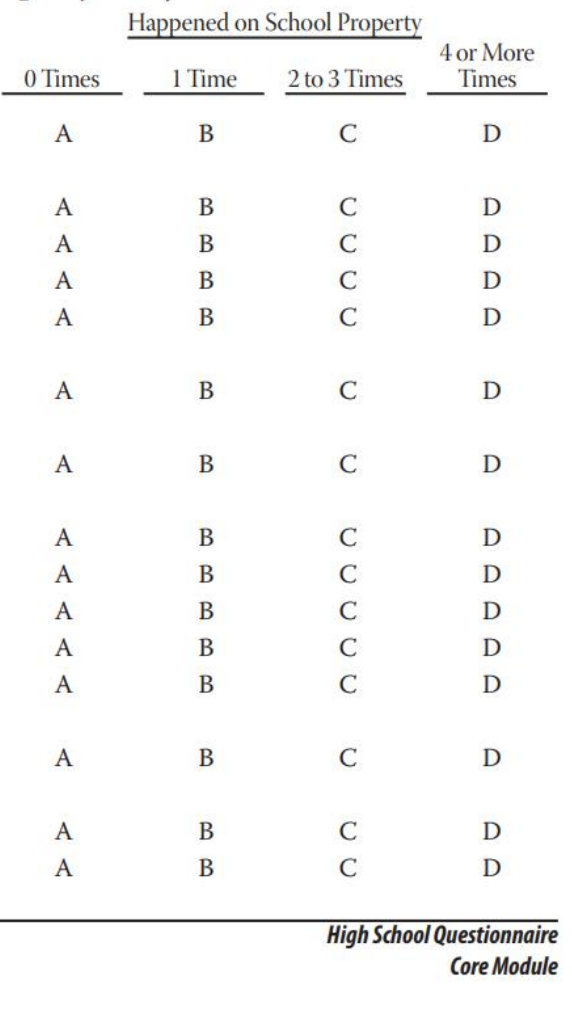




\section{A L I F OR N I A healthykids S U R VE Y}

\section{Core Module}

During the past 12 months, how many times on school property were you harassed or bullied for any of the following reasons? [You were bullied if you were shoved, hit, threatened, called mean names, teased, or had other unpleasant physical or verbal things done to you repeatedly or in a severe way. It is not bullying when two students of about the same strength quarrel or fight.]

105. Your race, ethnicity, or national origin

106. Your religion

107. Your gender (being male or female)

108. Because you are gay or lesbian or someone thought

$\underline{0 \text { Times }} 1$ Time 2 to 3 Times $\stackrel{\begin{array}{c}4 \text { or More } \\ \text { Times }\end{array}}{\text { C }}$
you were

109. A physical or mental disability

110. Any other reason

$\begin{array}{llll}\text { A } & \text { B } & \text { C } & \text { D } \\ \text { A } & \text { B } & \text { C } & \text { D } \\ \text { A } & \text { B } & \text { C } & \text { D } \\ \text { A } & \text { B } & \text { C } & \text { D } \\ & & & \\ \text { A } & \text { B } & \text { C } & \text { D } \\ \text { A } & \text { B } & \text { C } & \text { D }\end{array}$

111. During the past $\mathbf{1 2}$ months, how many times did other students spread mean rumors or lies about you on the internet (i.e., Facebook ${ }^{\mathrm{mw}}$, Instagram ${ }^{\mathrm{mw}}$, Snapchat ${ }^{\mathrm{mw}}$, email, instant message)?
A) 0 times (never)
B) 1 time
C) 2-3 times
D) 4 or more times

112. Do you consider yourself a member of a gang?
A) No
B) Yes

113. During the past 12 months, did you ever feel so sad or hopeless almost everyday for two weeks or more that you stopped doing some usual activities?
A) $\mathrm{No}$
B) Yes

114. During the past 12 months, did you ever seriously consider attempting suicide?
A) No
B) Yes

115. Did you eat breakfast today?
A) No
B) Yes

116. How many questions in this survey did you answer honestly?
A) All of them
B) Most of them
C) Only some of them
D) Hardly any 


\section{A L I F OR N I A healthykids SURVE Y Core Module}

117. Is your father, mother, or caretaker currently in the military (Army, Navy, Marines, Air Force, National Guard, or Reserves)?
A) No
B) Yes
C) Don't know

118. Which of the following best describes you? (Mark All That Apply.)
A) Heterosexual (straight)
B) Gay or Lesbian
C) Bisexual
D) Transgender
E) Not sure
F) Decline to respond 
Appendix B: Institutional Review Board Approval for Exempt Status

Date: $1-17-2021$

IRB \#: IRB-21-94

Title: Bidimensional Assessment of Youth Mental Health: Evaluation of the California Healthy Kids Survey as a Measure of Youth Subjective Well-Being.

Creation Date: 10-18-2020

End Date:

Status: Approved

Principal Investigator: Michael Hass

Review Board: Exempt

Sponsor:

\section{Study History}

Submission Type Initial Review Type Exempt Decision Exempt

\section{Key Study Contacts}

\begin{tabular}{lll}
\hline Member Michael Doria & Role Co-Principal Investigator & $\begin{array}{l}\text { Contact } \\
\text { doria100@mail.chapman.edu }\end{array}$ \\
\hline Member Michael Hass & Role Principal Investigator & Contact mhass@chapman.edu \\
\hline Member Michael Doria & Role Primary Contact & $\begin{array}{l}\text { Contact } \\
\text { doria100@mail.chapman.edu }\end{array}$ \\
\hline
\end{tabular}




\section{A L I F O R N I A healthy Rids $S$ U R V E Y}

\section{Social Emotional Health Module}

\section{SUPPLEMENT 1}

Please tell us how true each statement is of you ...

W1. I can work out my problems.

W2. I can do most things if I try.

W3. There are many things that I do well.

W4. There is a purpose to my life.

W5. My intelligence is something I cannot change very much.

W6. I understand my moods and feelings.

W7. I understand why I do what I do.

W8. I enjoy working together with other students on class activities.

W9. When I do not understand something, I ask the teacher again and again until I understand.

W10. I try to answer all the questions asked in class.

W11. When I try to solve a math problem, I will not stop until I find a final solution.

W12. I accept responsibility for my actions.

W13. I am looking forward to a successful career.

W14. When I make a mistake I admit it.

W15. I can deal with being told no.

W16. I feel bad when someone gets their feelings hurt.

W17. When I need help I find someone to talk with.

W18. I try to understand what other people go through.

W19. I have high goals and expectations for myself.

W20. I try to understand how other people feel and think.

W21. I can wait for what I want.

W22. Challenging myself will not make me any smarter.

W23. I don't bother others when they are busy.

W24. I think before I act.

W25. Each day I look forward to having a lot of fun.

W26. When I work in school groups, I do my fair share.

W27. I usually expect to have a good day.

W28. Overall, I expect more good things to happen to me than bad things.

$\begin{array}{llll}\text { A } & \text { B } & \text { C } & \text { D } \\ \text { A } & \text { B } & \text { C } & \text { D } \\ \text { A } & \text { B } & \text { C } & \text { D } \\ \text { A } & \text { B } & \text { C } & \text { D } \\ \text { A } & \text { B } & \text { C } & \text { D }\end{array}$

\section{A}

A

A

A

A

A

A

A

A

A

A

A

A

A

A

A

A

A

A

A

A

A

A

$\begin{array}{ll}\text { C } & \text { D } \\ \text { C } & \text { D } \\ \text { C } & \text { D }\end{array}$

B C D

B C D

B

C

D

$\begin{array}{lll}\text { B } & \text { C } & \text { D } \\ \text { B } & \text { C } & \text { D } \\ \text { B } & \text { C } & \text { D } \\ \text { B } & \text { C } & \text { D } \\ \text { B } & \text { C } & \text { D } \\ \text { B } & \text { C } & \text { D } \\ \text { B } & \text { C } & \text { D } \\ \text { B } & \text { C } & \text { D } \\ \text { B } & \text { C } & \text { D }\end{array}$

$\begin{array}{lll}\text { B } & \text { C } & \text { D } \\ \text { B } & \text { C } & \text { D } \\ \text { B } & \text { C } & \text { D } \\ \text { B } & \text { C } & \text { D } \\ \text { B } & \text { C } & \text { D } \\ \text { B } & \text { C } & \text { D } \\ \text { B } & \text { C } & \text { D } \\ \text { B } & \text { C } & \text { D }\end{array}$

Middle \& High School Questionnaire Social Emotional Health Module 


\section{A L I FOR N I A healthykids $S$ U R V Y}

\section{Social Emotional Health Module}

\section{SUPPLEMENT 1}

Please tell us how true each statement is of you...

\begin{tabular}{|c|c|c|c|}
\hline Not At All & A Little & Pretty Much & Very Much \\
\hline True of & True of Me & & True of $\mathrm{M}$ \\
\hline
\end{tabular}

W29. I try to work out my problems by talking or writing about them.

W30. There are some things I am not capable of learning. True of Me True of Me True of Me True of Me

$\begin{array}{llll}\text { A } & \text { B } & \text { C } & \text { D } \\ \text { A } & \text { B } & \text { C } & \text { D } \\ \text { A } & \text { B } & \text { C } & \text { D } \\ \text { A } & \text { B } & \text { C } & \text { D } \\ \text { A } & \text { B } & \text { C } & \text { D } \\ \text { A } & \text { B } & \text { C } & \text { D }\end{array}$

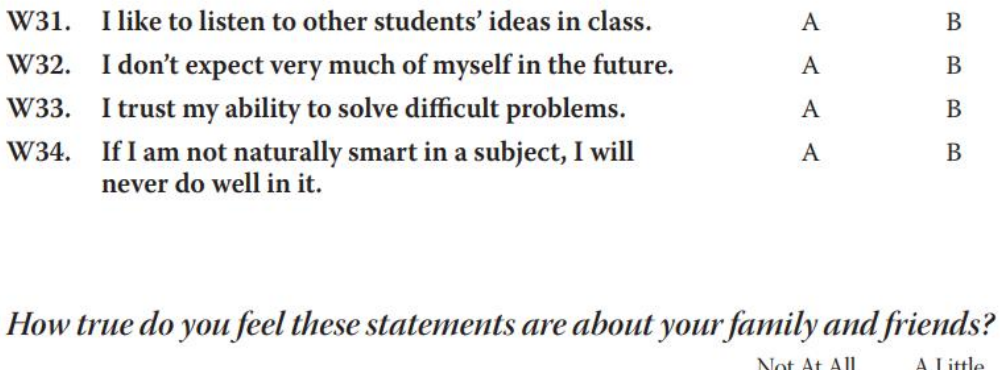

W35. My family members really help and support one Not At All
True A Little
True Pretty Much Very Much another.

$\begin{array}{llll}\text { A } & \text { B } & \text { C } & \text { D }\end{array}$

W36. There is a feeling of togetherness in my family.

W37. My family really gets along well with each other.

W38. I have a friend my age who really cares about me.

W39. I have a friend my age who talks with me about my problems.

W40. I have a friend my age who helps me when I'm having a hard time.

$\begin{array}{llll}\text { A } & \text { B } & \text { C } & \text { D } \\ \text { A } & \text { B } & \text { C } & \text { D } \\ \text { A } & \text { B } & \text { C } & \text { D } \\ \text { A } & \text { B } & \text { C } & \text { D } \\ & & & \\ \text { A } & \text { B } & \text { C } & \text { D }\end{array}$

Please tell us how strongly you feel the following emotion ...

Not At All A Little Somewhat $\begin{aligned} & \text { Quite } \\ & \text { A Lot }\end{aligned}$ Extremely

W41. Since yesterday, how much have you felt GRATEFUL?

$\begin{array}{lllll}\text { A } & \text { B } & \text { C } & \text { D } & \text { E }\end{array}$

$\begin{array}{lllll}\text { A } & \text { B } & \text { C } & \text { D } & \text { E }\end{array}$

W42. Since yesterday, how much have you felt THANKFUL?

A $\quad$ B $\quad$ C $\quad$ D $\quad$ E

W43. Since yesterday, how much have you felt APPRECIATIVE?

California Healthy Kids Survey O2016 CA Dept. of Ed.

Middle \& High School Questionnaire

Version MSHS20 - Fall 2016-Spring 2017

Social Emotional Health Module 


\section{A L I F ORN I A healthykids SUR VE Y}

\section{Social Emotional Health Module}

\section{SUPPLEMENT 1}

How do you feel right now?

\begin{tabular}{|c|c|c|c|c|}
\hline Not At All & A Little & Somewhat & $\begin{array}{l}\text { Quite } \\
\text { A Lot }\end{array}$ & Extremely \\
\hline A & B & C & D & E \\
\hline A & B & C & D & $\mathrm{E}$ \\
\hline A & B & $\mathrm{C}$ & D & E \\
\hline
\end{tabular}

W45. How much do you feel ACTIVE right now?

W46. How much do you feel LIVELY right now? 